\title{
Quantum Model of Inertia - Predictions - Confirmations, Consequences for Gravitation into Galaxies, and LCDM Cosmology Models
}

\author{
Claude Poher $^{1} \&$ Danielle Poher ${ }^{1}$ \\ ${ }^{1}$ Laboratoire Aurora, Chemin de la Bourdette, Toulouse, France \\ Correspondence: Claude Poher, Laboratoire Aurora, 33 Chemin de la Bourdette, F31400, Toulouse, France. Tel: \\ 33-0-562-177-896. E-mail: claude.poher@wanadoo.fr
}

Received: May 4, 2020

Accepted: July 26, 2020

Online Published: July 31, 2020

doi:10.5539/apr.v12n4p8

URL: http://dx.doi.org/10.5539/apr.v12n4p8

\begin{abstract}
We propose a Quantum model of Inertia using two main hypotheses, (i) existence of a general isotropic flux of Quanta (named "Universons", which differ from Gravitons), propagating at light velocity into the Universe, (ii) a very short time duration interaction of these Quanta with elementary massive matter particles, with temporary momentum transfer (really a $2 \pi$ phase shift of the wave function). Model parameters values are obtained from observations. The natural flux has Random Fluctuations in intensity and in direction, predicted to be the cause of Gravitational acceleration, a model of Gravitation is deduced. Predictions of the two models are confirmed by free fall of cold Neutrons; and by strong electrons accelerations into superconducting devices. A supplementary Cosmological acceleration $H_{0} c$, from Universe expansion, is predicted and confirmed by Astronomical observations. Galaxies rotation velocities are predicted from quantum fluctuations and $\mathrm{H}_{0} \mathrm{c}$ effect, solving the enigma that required Cold Dark Matter mass hypothesis. The cosmological concordance model has therefore to be strongly modified, and the isotropic flux expansion could also explain observations without "Dark Energy".
\end{abstract}

Keywords: Cdm Models, Dark Energy, Dark Matter, Gravitation, Inertia, Mond Paradigm

\section{Introduction, General Concept}

We propose a Quantum model of Inertia using Quanta named «Universons» to distinguish them from hypothetic «Gravitons», which are different. Our model is also the one of Quantum Gravitation by its consequences.

The motivation for the present work originated from Astronomical observations of galaxies rotation curves, suggesting that Gravitation is not, as proposed by Newton in 1687, an attractive force between two masses of matter, or insufficiently suggested by General Relativity, a local space-time local distortion. It should be a Quantum «pushing» momentum from different directions. That is to say, "pushing" the two masses of matter one towards the other.

This «Push from the Quanta» would be present everywhere, and it would act as well on an isolated object in Space. In this case, the object receiving the same push from all directions, no resultant effect would be observed.

The idea that Gravitation could be the result of a "bombardment" of subatomic entities is not a new one. Several authors have proposed, since long, models where an isotropic flux of fast moving quanta travel in the Universe and interact with matter. Such a model was proposed by Nicolas Fatio de Duillier, (1664 - 1753) when he was in relation with Isaac Newton. And Swiss physicist Georges-Louis Lesage suggested the same model, in 1758 to French Academy. These were "collisions models" with absorption (shadow effect) which are not compatible with observations. Stable planets orbits are effectively impossible with absorbed Quanta.

So, there is a clear difference between the «Universons model» we propose here and anterior ideas of this type. The difference lays into the original properties of the interaction of Universons, properties never described in the scientific literature. Into our model there is no quanta absorption, only momentum orientation change.

\section{Hypotheses of Our Model}

Two hypotheses are required for that model.

(i) - A huge isotropic flux of Gravitational Quanta (named here "Universons") propagating at light velocity into the whole Universe. These Quanta carry each a same positive tiny kinetic energy $E \mathrm{u}$. 
(ii) - There is an interaction of these quanta with all massive elementary particles of matter they cross. This interaction has two properties: firstly, it is temporary, with a very short duration $t$, during which the positive quantum momentum $E u / c$ is transferred to the matter particle; moreover there is not any absorption of the quanta energy after the interaction is finished. So the quantum recovers its freedom, and properties, after the interaction time has elapsed. The momentum direction of an interacting Quantum is changed in certain circumstances.

\section{Constraints from Physics, Methods of Presentation}

Quanta properties from our model are of course constrained by known laws of physics, as well as by known behaviour of Nature. These constraints will appear along the model development. However it seems useful to avoid interpretation errors right at beginning of that model presentation, even if that is anticipation of results.

For example, Universons all carry only a same $E u$ kinetic energy, not an electromagnetic one. This energy is positive therefore Universons momentum $E u / c$ is also always positive, this means they create a pushing effect on matter particles. Universons have no electric charge, their interaction being only concerned by mass, not by charge.

For clarity, we have developed the interaction consequences of known laws of physics into separate Appendixes, even if the corresponding properties have a direct influence onto the model development.

We have also used specific terms along the model presentation. For example "Capture" is the time period when a single Universon is interacting with a matter particle. This is not a real capture, the wave function of an Universon is much more extended than the one of a matter particle, according to Quantum Physics. As well "Re emission" is the operation by which the quantum interaction time has elapsed, and the quantum recovers its freedom. This is not either a real emission. We will show that the interaction shifts the wave function of the Universon by $2 \pi$ radians, which looks like a time delay $t$.

Should we use Quantum Physics?

Answering that question anticipates results from Appendix A, where the value of product $E u t$ is obtained thanks to $L$. de Broglie's quantum physics work, made half a century ago. So we show that $E u t=h$, the Plank's constant. This value is $4 \pi$ times larger than the Heisenberg's limit; for classical Physics use. Consequently, we are going here to consider trajectories of Gravitational Quanta at velocity c, before and after an interaction with matter particles, from the use of Classical Physics, with Relativity and Lorentz' relations.

\section{Model Presentation}

Let us consider two parallel reference frames \#1 and \#2 (Figure 1). They are classical, with 3 perpendicular axes. Frame \#1 is the one of a virtual observer at rest. He looks at the arrival of one incident Universon, from the natural flux. Frame \#2 is tied to an elementary particle of matter, of mass $M$, and speed $v$ in frame \#1, along the Ox axis of frame \#1. The speed of light $c$ is the Universons speed in the two reference frames. We define the two classical relativistic parameters:

$$
\begin{gathered}
\beta=v / c \\
\gamma=\left(1-v^{2} / c^{2}\right)^{-1 / 2}
\end{gathered}
$$

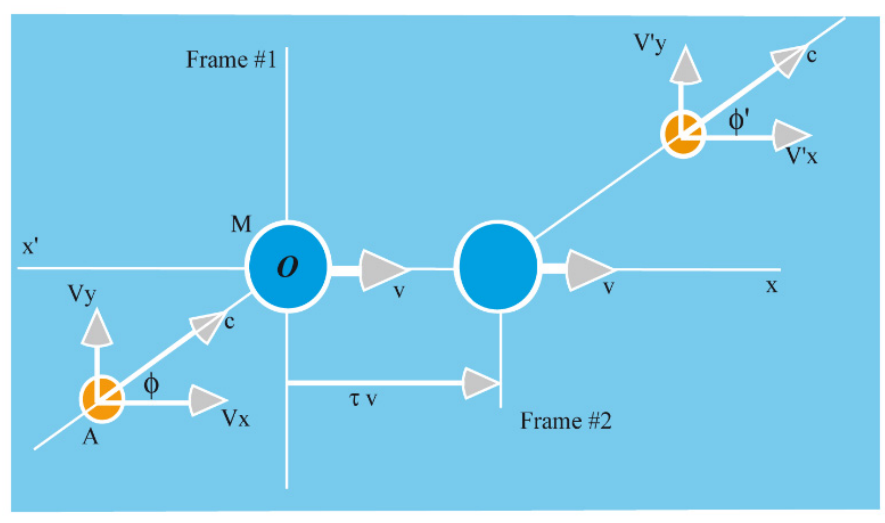

Figure 1. Interaction of a Gravitational quantum with a matter particle moving at constant velocity 
The momentum $P$ of the Universon, or the one of the matter particle, will have subscript 1 or 2 , according to the frame from where this momentum is observed. Moreover, this momentum, which is a vector, will be represented by its components along the 3 axes of each frame. So there will be one more subscript, $x, y$ or $z$ in order to show this.s í The rest energy of the Universon will be represented by $E u$ in each frame, with the corresponding subscript.

The direction of the positive, constant, low speed $v$ of the matter particle is supposed parallel to the Ox axis of each of the two frames. So, the transformation of momentum, observed in the two frames, will use the following Lorentz' relativistic physics relations:

$$
\begin{gathered}
P_{x 2}=\gamma\left(P_{x 1}-\beta E_{1} / c\right) \\
P_{y 2}=P_{y 1} \\
P_{z 2}=P_{z 1} \\
E_{2}=\gamma\left(E_{1}-c \beta P_{x l}\right)
\end{gathered}
$$

The interaction time

of the Universon, in frame \#2, is not the same when observed in frame \#1:

$$
\tau_{1}=\gamma \tau_{2}
$$

Moreover, as free Universons travel at constant speed $c$ in the two frames, one can say necessarily:

$$
P_{1}=E_{l} / c
$$

The 3 components of momentum $P_{1}$ of the Universon, in frame \#1, are tied to the incident trajectory of the Universon. Let us suppose that the Universon trajectory is in the $\mathrm{xOy}$ plane of frame \#1, as shown in Figure 1, with an angle between the Universon trajectory and the Ox axis, we can write:

$$
\begin{gathered}
P_{x l}=\left(E_{1} / c\right) \cos \phi \\
P_{y l}=\left(E_{1} / c\right) \sin \phi \\
P_{z l}=0
\end{gathered}
$$

\subsection{Interaction of Universons with a Matter Particle Moving at Constant Speed}

For our model, to be in conformity with the inertia principle, a constant speed particle of matter should not be perturbed by the existence of an isotropic, natural flux of Universons, interacting with it.

Let us therefore consider the interaction of a single Universon with an elementary particle of matter bearing a mass. This particle has a constant speed $v$ along axis $\mathrm{Ox}$ in frame \#1. The particle is at rest in frame \#2.

We have used a kind of "pedagogical" Figure 1 for representation of the interaction, with spheres and vectors even if the Universons and the matter particle should really be "Wave functions", not spheres but impossible to show this way.

For ease of understanding equations, this representation reveals to be "acceptable" (we are in classical physics here).

Therefore that figure should only be considered as a "presentation tool", not a reality.

The momentum and rest energy of the incident Universon, defined by expressions (8) to (11) in reference frame \#1, do not have the same values when observed from the moving particle, in reference frame \#2.

So, the particle of matter interacts with an incoming Universon A having different characteristics than the (8) to (11) ones. We have to use transformations (3) to (6) to know the values of the momentum and energy exchanged while the interaction is taking place:

$$
\begin{gathered}
P_{x_{2}}=\gamma\left\{\left(E_{1} / c\right) \cos \phi-\beta E_{1} / c\right\} \\
P_{y 2}=\left(E_{1} / c\right) \sin \phi \\
P_{z 2}=0 \\
E_{2}=\gamma\left\{E_{1}-c \beta\left(E_{1} / c\right) \cos \phi\right\}
\end{gathered}
$$

Expression (12) can be written:

$$
P_{x 2}=\left(\gamma E_{1} / c\right)(\cos \phi-\beta)
$$


Expression (15) becomes:

$$
E_{2}=\gamma E_{1}(1-\beta \cos \phi)
$$

At the very moment of the Universon capture by the particle of matter, we can suppose that its energy $E_{2}$ is changed into a mass increase $m$ of the particle, in such a way that the relativistic equivalence of mass and energy is satisfied:

$$
m=E_{2} / c^{2}
$$

Or:

$$
m=\left(\gamma E_{1} / c^{2}\right)(1-\beta \cos \phi)
$$

Moreover, the matter particle receives an increase of its momentum, because the impulses defined by (13), (14) and (16) are transferred to it integrally.

It is interesting to consider what should happen to the particle of matter if it would capture simultaneously another Universon, coming from a direction exactly opposed to the direction of the previous one. In this case we should consider the previous relations, but with an incidence angle $\phi+\pi$ instead of $\phi$ that would reverse the signs of $\sin \phi$ and of $\cos \phi$. So for this second Universon we would observe:

$$
\begin{gathered}
P_{x 2}=\left(\gamma E_{1} / c\right)(-\cos \phi-\beta) \\
P_{y 2}=-\left(E_{1} / c\right) \sin \phi \\
P_{z 2}=0 \\
E_{2}=\gamma E_{1}(1+\beta \cos \phi) \\
m=\left(\gamma E_{1} / c^{2}\right)(1+\beta \cos \phi)
\end{gathered}
$$

The momentum transferred to the particle of matter by the two interacting Universons, along axis Oy of reference frame \#2, defined by (13) and (21) are opposed and they cancel each other when observed macroscopically. Effectively, the particle interacts with a very large number of Universons from an isotropic flux, so there are numerous Universons interacting simultaneously from all the directions of space.

Expressions (17) and (23) tell us the value of the energy transferred to the particle of matter by two Universons with an opposed trajectory. Those energies are not equal.

However, if we consider the effect of these two Universons on the mass increase of the particle while they interact simultaneously, we have to add expressions (19) and (24), and then we get:

$$
m_{(19)}+m_{(24)}=2 \gamma E_{1} / c^{2}
$$

We observe that the total mass increase of the particle of matter is exactly the same as if two Universons of the same energy $E_{1}$ (the rest energy observed in frame \#1), were interacting with the same particle, at rest, in frame \#1. This is a curious but important result.

Let us stop for a moment our verification of the inertia principle compatibility, in order to consider the consequences of this fact.

\subsection{Proper Mass of a Matter Particle}

Expression (25) demonstrates that the simultaneous capture of two incident Universons, with opposed trajectories, induces a total mass increase of the matter particle, equal, if we ignore the $\gamma$ factor, to the mass increase induced by any two Universons captured when the particle is at rest. So, for the particle, being at rest or in uniform movement, does not change its mass increase, except by the $\gamma$ factor, which is precisely a known result of Relativity theory.

Moreover, the interaction of one Universon with a particle of matter has a finite duration, which is a constant time $\tau_{2}$ in frame $\# 2$.

Let us call $F_{u}$ the intensity of the natural flux of free Universons. This intensity is measured in particular units : Universons per second, per square meter, coming from the $4 \pi$ steradians.

Let us call $S$ the «specific capture (interaction) cross section » of Universons by particles of matter. This is not a surface, but « a surface per kilogram of matter particle mass». With these units, an elementary particle of matter of rest mass $M_{o}$ interacts simultaneously with $n$ Universons, during the capture time $\tau_{2}$ of one of them: 


$$
n=\tau_{2} S M_{o} F_{u}
$$

Each interacting pair of these $n$ Universons, with an opposed trajectory, induces a mass increase of the matter particle given by expression (25).

So, the total mass increase $M_{2}$ caused by all the $n$ Universons captured during time $\tau_{2}$ will be the product of (25) by $n / 2$ :

$$
M_{2}=\tau_{2} S M_{o} F_{u} \gamma E_{1} / c^{2}
$$

Replacing $\tau_{2}$ by its value (7), we get:

$$
M_{2}=\tau_{1} S M_{o} F_{u} E_{1} / c^{2}
$$

Now, when the capture time $\tau_{1}$ has elapsed, the first captured Universons are re-emitted, and immediately replaced by new interacting ones. So the total number of permanently captured Universons remains constant and equal to $n$. Finally, the total mass increase $M_{2}$ of the matter particle in reference frame \#2 remains constant on average, and evidently it must be equal to the observed, permanent, and constant, rest mass $M_{o}$ of the particle:

$$
M_{o}=\tau_{1} S M_{o} F_{u} E_{1} / c^{2}
$$

So, evidently:

$$
\tau_{1} S F_{u} E_{1} / c^{2}=1
$$

Expression (30) is one of the fundamental relations of the Universons model. It ties the parameters of the theory.

We might consider also that, with relation (30), we get another fundamental result:

$$
S \boldsymbol{F}_{\boldsymbol{u}}=\boldsymbol{c}^{2} / \boldsymbol{h}
$$

This expression tells us the total number of Universons permanently captured by a kilogram of matter, and permanently replaced by new captured ones, as they are re-emitted. This number is gigantic: $1.36 .10^{50}$.

According to (18) \& (26), relation (30) has an important meaning: the rest mass of an Universon captures only one Universon during the capture time (itself).

More than that, from previous relations, we see that, for matter at rest:

$$
M_{o}=n E_{l} / c^{2}
$$

This means that the rest mass of any particle of matter is made of the total energy of the simultaneously captured Universons.

These captured Universons are permanently replaced after being "captured" for a very short time.

Effectively, if the capture time $\tau$ was quite long, we should have already observed the fluctuations of the mass caused by the not perfect coincidence of capture and re-emission of the pairs of Universons.

This behaviour is only acceptable if the capture time is sufficiently short so as the uncertainty principle is macroscopically respected, concerning the conservation of the energy and momentum of matter and the Universons.

Nevertheless any rest mass $M_{o}$ of any particle of matter is subject to tinny and very rapid random fluctuations.

These fluctuations were predicted by Louis de Broglie, into his "double solution” theory.

These fluctuations follow the Laplace-Gauss's distribution, as it is the case for all Quantum Physics phenomena, with the corresponding properties. For example, about $99 \%$ of the time, the rest mass of a matter particle fluctuates between $M_{o}-3 \sigma$ and $M_{o}+3 \sigma$ with $\sigma=\left(M_{o}\right)^{1 / 2}$ and a frequency of these fluctuations proportional to $n / \tau$.

Moreover, we have shown that the observed mass $M_{v}$ of a matter particle of rest mass $M_{o}$ observed from reference frame \#1, when the particle moves at constant speed $v$ relative to this frame, is according to Relativity theory:

$$
M_{v}=\gamma M_{o}
$$

That is simply the result of the capture time transformation between the two frames (7):

$$
\tau_{1}=\gamma \tau_{2}
$$

This shows that the model is correct from the relativistic point of view.

But let us now return to the main verification process of the compatibility of the model with the inertia principle. 


\subsection{Re-Emission of Captured Universons by a Matter Particle in Uniform Movement}

Now, we are considering a new reference frame \#3, which is frame \#2 moving at constant speed $-v$ along Ox axis. Evidently, frames \#1 and \#3 are identical, but this will avoid errors on the subscripts in our calculations.

Each captured Universon is re-emitted at the end of the capture time $\tau$ in such a way that the average particle mass remains constant. This means that, in frame \#2, energy $E_{2}$ must be exchanged between the particle of matter and the re-emitted Universon. Consequently, the momentum defined by (13), (14) and (16) is transferred to the Universon, such that the average macroscopic movement of the matter particle is not perturbed. Those are the necessary conditions imposed by the inertia principle.

These energy and momentum, transferred to the Universon, will be observed from reference frame \#3, so that we will be able to compare the characteristics of the incident and re-emitted Universon in the same frame \#1. The transformation of these quantities from frame \#2 to frame \#3 uses expressions (3) to (6), with a reverted sign for $\beta$ because speed $v$ of frame \#3 is negative:

$$
\begin{gathered}
P_{x 3}=\gamma\left(P_{x 2}+\beta E_{2} / c\right) \\
P_{y 3}=P_{y 2} \\
P_{z 3}=P_{z 2} \\
E_{3}=\gamma\left(E_{2}+c \beta P_{x 2}\right)
\end{gathered}
$$

Replacing the terms defined by (13), (14) and (16) we obtain:

$$
\begin{gathered}
P_{x 3}=\gamma\left\{\left(\gamma E_{1} / c\right)(\cos \phi-\beta)+\beta \gamma E_{1}(1-\beta \cos \phi) / c\right\} \\
P_{y 3}=\left(E_{1} / c\right) \sin \phi \\
P_{z 3}=0 \\
E_{3}=\gamma\left\{\gamma E_{1}(1-\beta \cos \phi)+c \beta\left(\gamma E_{1} / c\right)(\cos \phi-\beta)\right\}
\end{gathered}
$$

Simplifying (37), we get:

$$
P_{x 3}=\left(E_{1} / c\right) \cos \phi
$$

Simplifying (40):

$$
E_{3}=E_{1}
$$

The trajectory of the re-emitted Universon is defined by a new angle $\phi$ :

$$
\begin{aligned}
& P_{x 3}=\left(E_{3} / c\right) \cos \phi^{\prime} \\
& P_{y 3}=\left(E_{3} / c\right) \sin \phi^{\prime}
\end{aligned}
$$

Considering the meaning of relations (38), (39) and (42) to (44), it becomes evident that:

- On the one hand, the re-emitted Universon has the same energy as the incident one in frames \#1 and \#3 which are strictly identical.

- On the other hand, the incidence and re-emission angles $\phi$ et $\phi$ ' are equal, which means that the Universon flux remains isotropic when interacting with matter moving at constant speed.

We can therefore affirm that the interaction of matter in uniform movement with the natural flux of Universons does not perturb the matter movement, and does not change the isotropy of the Universons flux.

So, now we have verified that this Universons model is not in conflict with Inertia principle.

This is not sufficient to prove that the model is correct, because there must also be a compatibility of the theory with two more phenomena:

A - On the one hand, the behaviour with accelerated matter (Newton's Inertia law).

B - On the other hand, we should also look at the behaviour when two bodies of matter are acting on each other (Newton's gravitational law).

\section{We will undertake now demonstrations of behaviour A which concerns Inertia.}

Then from the Inertia model properties will then come the Gravitation model into Appendix H. 
There is one important fact predicted by the Universons theory which must be taken into account for our verifications: particles of matter are submitted to random fluctuations of their rest mass, and momentum, caused by their permanent interaction with the natural flux of Universons. Louis de Broglie considered himself that hypothesis during the seventies (Appendix A).

\subsection{Interaction of Universons with Accelerated Matter Particle}

Let us now consider the interaction of a single Universon, with a particle of matter, accelerated along the $\mathrm{Ox}$ axis of frame $\# 1$. The particle acceleration $A$ is supposed constant, and frame $\# 2$, where the matter particle remains at rest, is supposed starting at frame \#1 position, at the instant of the Universon interaction.

The pedagogical Figure 2 helps understanding this situation, with the two frames superposed.

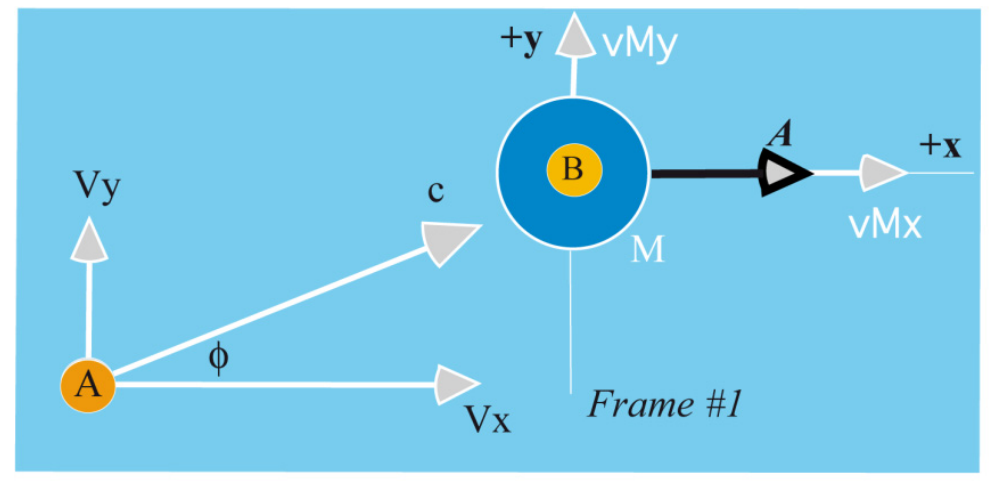

Figure 2. Interaction of a Gravitational quantum with an accelerated matter particle at capture time

The incident Universon A is captured in B at the start of frame $\# 2$ acceleration with the particle $\mathrm{M}$.

The incident Universon A has the following momentum components in reference frame \#1:

$$
\begin{gathered}
P_{l}=E_{l} / c \\
P_{x l}=\left(E_{1} / c\right) \cos \phi \\
P_{y l}=\left(E_{1} / c\right) \sin \phi \\
P_{z l}=0
\end{gathered}
$$

When the Universon is captured in position B, its energy $E_{l}$ is changed into a mass increase $m$ of the matter particle. In this capture process the Relativistic equivalence of mass and energy is satisfied:

$$
m=E_{1} / c^{2}
$$

The matter particle recoils because the momentum defined by (46), (47) and (48) is integrally transferred to it. It is interesting to consider what happens with another incident Universon, coming from a direction directly opposed to the direction of the previous one. In this case we should consider an incidence angle equal to $\phi+\pi$ instead of $\phi$ and this would reverse the signs of $\sin \phi$ and $\cos \phi$. In this case we would get:

$$
\begin{gathered}
P_{x l}=-\left(E_{1} / c\right) \cos \phi \\
P_{y l}=-\left(E_{1} / c\right) \sin \phi \\
P_{z l}=0 \\
m=E_{1} / c^{2}
\end{gathered}
$$

We observe that the momenta of the two Universons, with opposed trajectories, would compensate exactly so that the particle of matter would not move. This is true for any pair of Universons with opposed trajectories.

It is also interesting to consider what happens when two interacting Universons are simultaneously coming from symmetrical directions relatively to $+x$. In this case we should consider an incidence angle equal to $-\phi$ instead of $\phi$ and this would reverse only the sign of $\sin \phi$ and not the one of $\cos \phi$. Then we would get:

$$
P_{x l}=\left(E_{1} / c\right) \cos \phi
$$




$$
P_{y l}=-\left(E_{1} / c\right) \sin \phi
$$

We observe that the momenta transferred to matter by the two Universons with symmetrical trajectories would compensate exactly in the $\mathrm{y}$ direction, but would add in the $\mathrm{x}$ direction of acceleration.

Exactly at the beginning of the capture time $\tau$ we suppose that an external cause creates the acceleration $A$ of the particle of matter which begins to move along axis $\mathrm{x}$.

The observer remains in frame \#1. Effectively, the Lorentz's equations that we used previously are not adapted to accelerated frames.

So we are going to suppose that the capture time $\tau$ of the Universon by the matter particle is observed from this \#1 frame.

The whole elementary matter particle is supposed accelerated by an external cause, from the beginning of time count (time zero). And this is also supposed here to be exactly the beginning of the Universon capture.

As soon as it is captured, the Universon "disappears", and is changed into a part $m$ of the matter particle mass. And we are going to consider that this mass element $m$ is now the bearer of the energy and of the momentum of the captured Universon.

This is of course a purely pedagogical method for studying the interaction, because nothing distinguishes that mass element, from others. This simple method gives correct results and is easy to understand.

Thus, the elementary matter particle mass element $m$ has the following momentum and energy at instant $\mathrm{t}=0$, when the Universon has just been captured:

$$
\begin{gathered}
P_{x l}=\left(E_{1} / c\right) \cos \phi \\
P_{y l}=\left(E_{1} / c\right) \sin \phi \\
P_{z l}=0 \\
m=E_{l} / c^{2}
\end{gathered}
$$

( previous relations 46 to 49 )

The total energy $E_{m 0}$ of the mass element $m$ is expressed by the following relation at instant zero:

$$
E_{m 0}=m c^{2}
$$

Then, during the capture time $\tau$ the matter particle and its mass element $m$ are accelerated by an external cause along the $\mathrm{x}$ axis of frame \#1.

Consequently, their speed increases versus time. And their momentum and kinetic energy increase accordingly.

\section{Now let us consider instant $t=\tau$ in frame \# 1, just before the Universon re-emission.}

We are now going to look at previous quantities at the end of capture time $\tau$ just before the Universon is re-emitted. The variables indices 1 become $1 \tau$ for clarity.

The matter particle is moving now at speed:

$$
v=A \tau
$$

in frame \#1, along the $\mathrm{x}$ axis.

In relativistic physics, momentum $P$ acquired by a matter particle of mass $m$ moving at speed $v$ is given by expression:

$$
P=m \gamma v
$$

where the parameter $\gamma$ has the value defined by expression (2). Moreover, according to (2), and (49) and (57) we can write:

$$
P=(\beta \gamma) E_{1} / c
$$

The total energy $E$ of this same matter particle is given by the following expression:

$$
E=\gamma m c^{2}
$$

And the kinetic energy $E_{c}$ of this particle is expressed by:

$$
E_{c}=m c^{2}(\gamma-1)
$$

In those expressions, let us recall that the mass $m$ is the one caused by the Universon capture and defined by expression (49): 


$$
m=E_{1} / c^{2}
$$

So, the mass element $m$ of the elementary matter particle has the following components of its momentum, and the following total energy at the instant $\mathrm{t}=\tau$ in frame \# 1, just before the Universon re-emission:

$$
\begin{gathered}
P_{x I \tau}=\left(E_{1} / c\right)(\cos \phi+\beta \gamma) \\
P_{y l \tau}=\left(E_{1} / c\right) \sin \phi \\
P_{z I \tau}=0 \\
E_{m 1 \tau}=\gamma E_{l}
\end{gathered}
$$

And exactly after this instant, the Universon is re-emitted and the mass increase $m$ "disappears". $\backslash$ But we must not forget that the matter particle captures and re-emits Universons permanently. And this is the reason why the matter particle mass remains constant on average. So the mass element $m$ does not simply disappear, it is replaced by another one, created by the capture of another Universon, other mass element which is identical, and which is taking care of the momentum and kinetic energy.

\subsection{Re-Emission of the Universon by the Accelerated Matter Particle}

At end of the capture time, the previously captured Universon recovers its freedom.

We know, by experiments, that the total average mass of the matter particle does not change, and that its average kinetic energy is the one predicted in the absence of interaction with Universons.

The Universon re-emission is represented on Figure 3. The observer remains in frame \#1 as previously.

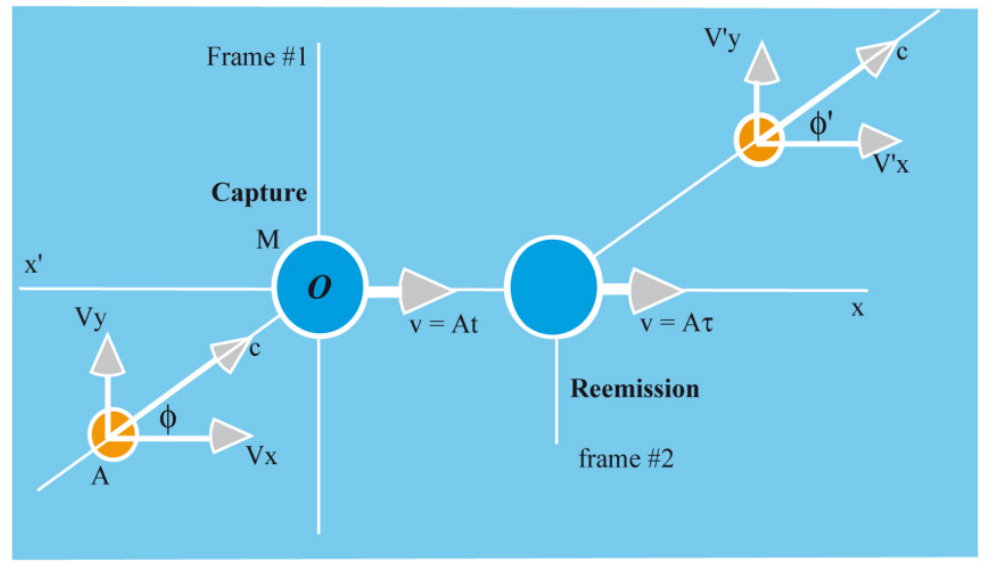

Figure 3. Interaction of a Gravitational quantum with an accelerated matter particle at Re-Emission time

As the Universon interaction with a matter particle does not change the average mass of matter, and does not change its final kinetic energy, it is essential that the re-emitted Universon energy $E_{\tau}$ be equal to:

$$
E_{\tau}=E_{m 1 \tau}=\gamma E_{1}
$$

The corresponding momentum $P_{\tau}$ is equal to:

$$
P_{\tau}=E_{\tau} / c=\gamma E_{l} / c
$$

Precisely, the Universons re-emission must not be the cause of a supplementary modification of the matter particle speed. This implies necessarily:

$$
P_{\tau}=P_{x l \tau}=\left(E_{1} / c\right)(\cos \phi+\beta \gamma)
$$

If we call $\phi$ ' the re-emission angle of the Universon in frame \#1, according to figure 3, we know that, by definition:

$$
P_{\tau}=\left(E_{\tau} / c\right) \cos \phi
$$

So, with (59 - 1) and (59 - 2):

$$
P_{\tau}=\gamma\left(E_{l} / c\right) \cos \phi '=\left(E_{l} / c\right)(\cos \phi+\beta \gamma)
$$


Which simplifies the following way:

$$
\cos \phi^{\prime}=(1 / \gamma) \cos \phi+\beta
$$

However, we know that $\beta=v / c$ with a speed $v=A \tau$ (57) which is always extremely small, whatever the value of the acceleration $A$ because the capture time $\tau$ is extremely brief. In these conditions, the value of the parameter $\gamma$ :

$$
\gamma=\left(1-v^{2} / c^{2}\right)^{-1 / 2}
$$

Is always equal to one with an error inferior to $10^{-39}$ and equation (59 - 6) can be simplified:

$$
\cos \phi '=\cos \phi+A \tau / c
$$

The expressions system defining the Universon re-emission conditions become:

$$
\begin{gathered}
P_{x l \tau}=\left(E_{1} / c\right) \cos \phi \\
P_{y 1 \tau}=\left(E_{1} / c\right) \sin \phi \\
P_{z l \tau}=0 \\
E_{m 1 \tau}=E_{1}
\end{gathered}
$$

In frame \#2, tied to the accelerated matter particle, the momentum and the kinetic energy of the particle are nil. Consequently, relations (60) represent the characteristics of the re-emitted Universon as seen by the observer situated in frame \#1.

Let us examine the direction of the Universon re-emission by comparing the angles $\phi$ of capture and $\phi^{\prime}$ of re-emission, both measured from the axis $\mathrm{x}$ in frame $\# 1$.

According to definition (59 - 7) let us recall that these angles are tied by expression:

$$
\cos \phi^{\prime}=\cos \phi+A \tau / c
$$

\section{Interpretation of These Results}

Interpretation of relations (59 - 7) and (60) reveals several facts:

Angles of incidence $\phi$ and of re-emission $\phi^{\prime}$ of the Universons are not equal. There exists an anisotropy of the re-emitted flux of captured Universons.

The momentum communicated to the accelerated particle of matter by the Universons interaction, in the direction opposed to the acceleration, is different than in the acceleration direction.

It suffices effectively to compare expressions (46) and (60 -1) to draw that conclusion.

This explains the inertia effect, and the need to exert a force on matter in order to be able to accelerate it. More calculation about that below.

This difference between capture and re-emission momenta manifests itself the same way in all space around the particle.

The anisotropy of the re-emitted flux of captured Universons, by accelerated matter, concerns all space around the particle of matter. This anisotropy has a rotation symmetry around the acceleration direction.

Compensation of the momentum transferred to matter, perpendicularly to the acceleration direction, by interaction with the Universon flux, does not appear Universon by Universon, but from pairs of captured Universons with opposed or symmetric incident trajectories, according to the acceleration direction.

Conservation of energy, and the one of momentum, are satisfied only at macroscopic scale, this means on average. The uncertainty principle authorizes this behaviour if the capture time of the Universons pairs is sufficiently short, which is always the case.

Taking into account the fact that, for all practical acceleration values, $A \tau / c<<<1$ which means that $\gamma=1$, expression (62) becomes:

$$
\cos \phi^{\prime}=\cos \phi+A \tau / c
$$

Now, let us consider the solid angle $\Omega$ ' defined by a cone with the half summit angle $\phi$ ' because the interaction is symmetric around the direction of the acceleration. The axis of this cone is the acceleration direction.

When $\phi=\pi$ then, expression (61) can be written: 


$$
\cos \phi^{\prime}=-1+A \tau / c
$$

From definition of solid angle:

$$
\Omega^{\prime}=2 \pi\left(1-\cos \phi^{\prime}\right)
$$

With (62) we obtain:

$$
\Omega^{\prime}=4 \pi-2 \pi A \tau / c
$$

This is the full sphere plus the solid angle:

$$
\Omega^{\prime}=-2 \pi A \tau / c
$$

In this very small solid angle $\Omega$ ', situated in the opposite direction of the acceleration, the accelerated particle of matter does not re-emit any captured Universon.

\section{This explains how the re-emitted Universons flux can be anisotropic.}

In expression (61), if $\phi=0$ then:

$$
\cos \phi^{`}=1+A \tau / c
$$

But, as $A \tau / c$ is positive, this expression is impossible, because the cosine of the re-emission angle cannot be larger than one. Interpretation of this fact is evidently that, in a very small solid angle:

$$
\Omega=2 \pi A \tau / c
$$

Situated in the direction of the acceleration, around $\phi=0$, the accelerated particle of matter does not capture any Universon coming from this solid angle.

These not-captured Universons continue their trajectory, as if matter was not there. So, in the direction of the acceleration, entirely inside the solid angle $\Omega$, the incident, natural flux of Universons, is not perturbed.

We can write expression (63) the following way:

$$
2 \pi\left(1-\cos \phi^{\prime}\right)=2 \pi(1-\cos \phi)-2 \pi A \tau / c
$$

Or, according to (63):

$$
\Omega^{\prime}=\Omega-2 \pi A \tau / c
$$

This expression shows that for an incident solid angle $\Omega=4 \pi A \tau / c$ the re-emission solid angle is only $\Omega^{\prime}=2 \pi$ $A \tau / c$ or two times less.

But we already know that, for all Universons coming in the solid angle $\Omega=2 \pi A \tau / c$ there is no capture. This means that they simply continue their trajectory and emerge in the solid angle $\Omega^{\prime}=2 \pi A \tau / c$;

However in this same emergence solid angle, there are also the Universons re-emitted after capture in the periphery of the incident solid angle $\Omega=4 \pi A \tau / c$.

So the OUTPUT flux of Universons, from the accelerated particle of matter, in the direction of the acceleration, and only in the solid angle $\Omega^{\prime}=2 \pi A \tau / c$ is always LARGER, than in the opposite direction, where the captured Universons are not re-emitted.

Considering facts $\# 5$ and $\# 7$ about the anisotropy of the interaction with an accelerated particle of matter, there are two particular, very small solid angles $\Omega=2 \pi A \tau / c$, of the same value, to consider. Both solid angles have the same axis, which is the acceleration direction, but they are opposed by their summit. One of these two solid angles is opened towards the front, the other one towards the rear.

In the front solid angle, the output flux of Universons is increased. In the rear solid angle, incident Universons are not captured.

We can consider a macroscopic mass of matter we are pushing by a force to accelerate it. All the elementary particles of that matter interact with the isotropic flux of Gravitational quanta. These interactions create an anisotropy (concentration of trajectories) of re-emitted quanta in the direction of acceleration. This "jet" of momentum bearers looks like the jet of gas from a rocket thruster. And it has the same propelling effect. Each matter particle is therefore pushed backwards by the momentum ejection. So the mass of matter and the interactions react by creating a backwards force, equal and opposed to the pushing force we exert (Action and reaction are equal). 
This is the way inertia acts really upon matter.

\subsection{Calculation of the Inertia Force}

According to (59 - 7) expression (60 - 1$)$ can be written:

$$
P_{x l \tau}=\left(E_{1} / c\right)(\cos \phi+A \tau / c)
$$

We know that this is the momentum transferred to the particle of matter by the re-emitted Universon, with a negative sign (in the direction $-\mathrm{x}$ ).

Let us compare this momentum with the one transferred to the particle of matter, in the $+\mathrm{x}$ direction, by the captured Universon. It was given by expression (46):

$$
P_{x 10}=\left(E_{1} / c\right) \cos \phi
$$

So now, by subtracting directly (46) from (70) we get the total momentum transferred to the matter particle, along the minus direction of the $\mathrm{x}$ axis, by the interaction of a single Universon:

$$
\Delta P_{x}=\left(E_{1} / c\right)(A \tau / c)
$$

The residual momentum (71) impedes the acceleration of the matter particle. This fact justifies the inertia effect, and the need to exert an external force in order to accelerate the matter particle.

The elementary force $\delta f$ that must be applied to the element of mass $m$ of the matter particle, in order to compensate the back momentum delivered by the interaction of a single Universon during time $\tau$, must be in principle, such that:

$$
\delta f=\Delta P_{x} / \tau=E_{1} A / c^{2}
$$

We are going to verify that this is correct.

In reality, we want to verify that this theory is compatible with Newton's law of Inertia.

So, we have to determine the value of the force, acting on the accelerated matter particle by the difference in linear momenta induced by the Universons interaction. The particle of matter has a total rest mass $\mathrm{M}_{0}$.

Let us call $F_{u}$ the intensity of the natural isotropic Universons flux, as previously. This flux is again expressed in Universons per second, per square meter, in the $4 \pi$ steradians.

This incident flux $F_{u}$ is isotropic, so the partial flux $\delta F(\phi, \psi)$ per steradian, in a direction defined by angles $\phi$ and $\psi$ is equal to:

$$
\delta F(\phi, \psi)=F_{u} / 4 \pi
$$

We will consider incident Universons, coming from direction $\phi, \psi$ where the angle $\phi$ is, as previously, measured in the $\mathrm{xOy}$ plane, and angle $\psi$ in the $\mathrm{yOz}$ plane.

Again, let us call $S$ the specific capture cross section of matter for the Universons interaction. So the flux $\delta F_{c}(\phi, \psi)$ of the captured Universons, coming in the direction $\phi, \psi$ is given by:

$$
\delta F_{c}(\phi, \psi)=S M_{0} F_{u} / 4 \pi
$$

This flux is expressed in captured Universons per second and per steradian, in the $(\phi, \psi)$ direction.

The number of Universons $\delta N(\phi, \psi)$ simultaneously captured, from this direction, during the capture time $\tau$ of one of them is equal to:

$$
\delta N(\phi, \psi)=\tau \delta F_{c}(\phi, \psi)=\tau S M F_{u} / 4 \pi
$$

Each one of these captured Universons transfers, to the particle of matter, when re-emitted, a supplementary momentum, in the direction opposed to the acceleration, which value is given by (71) copied here:

$$
\Delta P_{x}=\left(E_{1} / c\right)(A \tau / c)
$$

For each re-emitted Universon, the elementary force $\delta f$ exerted on the particle of matter, at the end of time $\tau$ is simply equal to $\Delta P_{x} / \tau$ :

$$
\delta f=\Delta P_{x} / \tau=A E_{1} / c^{2}
$$

So, the $\delta N(\phi, \psi)$ captured Universons during this time $\tau$, coming from the direction $(\phi, \psi)$ are exerting a total force, which is given by the product : $\delta f \delta N(\phi, \psi)$. 
The total force acting on the particle of matter for all the Universons coming from all the directions of space is obtained by integrating the value of this product in all space. This means that, by varying angle $\phi$ from 0 to $\pi$, and angle $\psi$ from 0 to $2 \pi$, we get:

$$
\text { Force }=(2 / \pi) \int_{\phi=0}^{\phi=\pi} \int \psi=0{ }^{\psi=2 \pi} \tau S M_{0} A E_{l} F_{u} /\left(4 \pi c^{2}\right) \delta \phi \delta \psi
$$

Finally:

$$
\text { Force }=\tau S M_{0} A E_{I} F_{u} / c^{2}
$$

But, from (30) we already know that:

$$
\tau S F_{u} E_{1} / c^{2}=1
$$

So, expression (79) becomes:

$$
\text { Force }=M_{0} \text { A }
$$

That is simply the well known Newton's Inertia law.

So, the Universons model is compatible with the Galileo's Inertia principle AND with the Newton's Inertia law.

\subsection{A Simpler Method for the Force Determination}

We have shown (76) that no Universon is re-emitted in a solid angle $\Omega^{\prime}=-2 \pi A \tau / c$, opposed to the direction of the acceleration. Macroscopically speaking, this means that incident Universons, coming from the direction opposite to the acceleration direction, into this solid angle, transfer to the matter particle a momentum, opposed to the direction of the acceleration, which is not compensated.

Moreover, incident Universons coming in the direction of the acceleration, in a solid angle $\Omega=2 \pi A \tau / c$, which axis is the direction of the acceleration, are not captured. Macroscopically speaking, this means that the re-emitted Universons in the direction of the acceleration direction, into the same solid angle, transfer to the matter particle a momentum, opposed to the direction of the acceleration, which is not compensated.

So the solid angle value $\Omega=2 \pi A \tau / c$ acts two times on the momentum transferred macroscopically to matter, in the opposite direction of the acceleration.

Expression (75) gives the average number of Universons captured, per steradian, during time $\tau$ in the $(\phi, \psi)$ direction. So the product of expression (82) by two times the value of the solid angle $\Omega=2 \pi A \tau / c$, should be the average number $N_{\Omega}$ of Universons exchanging a momentum in these solid angles:

$$
\begin{gathered}
N_{\Omega}=2 \Omega \delta N(\phi, \psi) \\
N_{\Omega}=A \tau^{2} S M_{0} F_{u} / c
\end{gathered}
$$

Each one of these Universons transfers to the particle of matter a momentum $E_{1} / c$ and the force is equal to the momentum divided by time duration $\tau$ so:

$$
\text { Force }=N_{\Omega} E_{1} / c \tau=A \tau S E_{1} M_{0} F_{u} / c^{2}
$$

But, from (30) we know that:

$$
\tau S F_{u} E_{1} / c^{2}=1
$$

So:

$$
\text { Force }=A M_{0}
$$

Which is as correct as (80).

This means that, in order to obtain the force acting on an accelerated particle of matter, it suffices, macroscopically, to determine the number of Universons captured in the solid angle $\Omega=2 \pi A \tau / c$, and then to multiply this number by $2 E_{1} / \tau c$. And finally, take into account fundamental expression (30).

\section{Prediction of Quantum Physics Properties from Inertia Model}

Particles of matter interact permanently with a huge number of Universons from the natural isotropic flux each second. We will show, from a specific Appendix C, the model parameters values, obtained from observations. Nevertheless from these appendixes we know that each matter particle interacts permanently with millions even billions of quanta during each capture time $\tau$, which is very brief as we will show. We are sorry not being able to 
present the different appendixes results into a simple logical order right now, because results from each one have consequences upon the methods and results of almost all others.

\subsection{The First Quantum Physics Property of Our Model Comes from the Number of Individual Interactions}

Effectively, it is easy to understand that the natural quanta interactions with matter particles are quite numerous and fully random. So matter particles are permanently agitated around their average position even when they are at rest. This is similar to a very tiny Brownian agitation, invisible to our instruments because of its average very low amplitude $\left(10^{-8}\right.$ Kelvin) and its high frequency (about $10^{20}$ Hertz).

Louis de Broglie had the intuition of that agitation, from an invisible milieu, he named "the thermostat", by analogy with thermodynamics reasoning. He participated to the elaboration of Quantum Physics almost a century ago, and tried to bring physical meaning to the wave function $\psi$ of matter particles and of photons.

We use therefore L. de Broglie's relations from his "Double solution theory" to get the value of product $E u \tau=h$ from quantum physics. This is detailed into Appendix A.

That fundamental relation is the one that allows us to use classical physics and Lorentz'transforms into our model. Most probably our model should also benefit from Quantum Physics direct approach in the future.

This Appendix A is also a first step towards to the evaluation of our model parameters.

\subsection{The Second Quantum Physics Property of Our Model Comes Also from That Random Distribution of Interactions with Matter Particles}

In the Universe, all matter is accelerated by Gravity. Therefore, all accelerated matter particles re-emit Universons with non-isotropic distribution of momenta in the acceleration directions.

As the captures and re-emissions are randomly distributed along time, and because the directions of accelerations of matter particles are also randomly distributed in different directions of space, the general isotropic flux of gravitational quanta has also a permanent randomly fluctuating component in amplitude and in direction.

There are also of course the quantum fluctuations of the quantum flux itself. All fluxes of this kind, such as a flux of light made of photons, are always fluctuating around an average amplitude, with a Gaussian distribution of these fluctuations.

The proper characteristics of fluctuations of the natural isotropic Quantum flux of Universons are presented into Appendix H. This has strong consequences onto the Gravity model we will discuss here under.

\section{Quantum Gravitation Model}

This model is presented into Appendix $\mathbf{H}$ from the fluctuations presented into Appendix G.

Our model shows that Universons from a Quantum fluctuation are dispersed by a mass of matter, because each fluctuation duration is much shorter than the interaction duration $\tau$. Therefore Universons from the fluctuation are mixed with many other Universons from the isotropic flux, as a minority, and are re-emitted like the majority.

So all fluctuations disappear really downstream. This way, on Earth for example, the average incident flux intensity is LARGER from the ZENITH direction than from the NADIR one, even if Universons from fluctuations are never absorbed by Earth matter.

Moreover, our model predicts (Appendix J) that the Gravitational acceleration value follows Newton's law only for interplanetary distances. Into galaxies, where Newton's law acceleration value becomes very small, the real Gravitational acceleration value increases; which gives a constant orbital velocity versus the galactic radius.

The model predicts (Appendix D) that there is also a "Cosmologic constant tiny acceleration" $H_{0} c$ caused by Universe expansion.

This prediction is confirmed by measurements of interplanetary spacecraft accelerations (Appendixes $\mathbf{E}$ and $\mathbf{F}$ ) so this has an influence on galaxies dynamic behaviour, which is also observed (Appendix K).

\section{Predictions of Parameters Values of Our Models (Appendix C)}

Parameters values for our models of Inertia and of Gravitation are tied together by mathematical relations we have presented in previous equations and which are also derived from other laws into Appendix $\mathbf{H}$.

So, in order to be able to get the real model parameters values, at least two of these parameters should be measured into a laboratory or from a known physics constant.

The laboratory determination of $E u$, the kinetic energy of a single Universon, is presented into Appendix B, from the free fall observation of cold Neutrons, into the Earth Gravity acceleration field, by Nevizhevsky \& al (2002) 
into the Laue-Langevin Institute in Grenoble (France).

This, added to the relation $E u \tau=h$ from Appendix A, gives the opportunity to calculate all other parameters values into Appendix $\mathbf{C}$. These values are the following;

\begin{tabular}{|c|c|}
\hline REST ENERGY OF A & Eu $=8.5 .10^{-21}$ Joule \\
\hline SOTROPIC FLUX OF UNIVERSONS & $\mathrm{F}=6.3 .10^{80} \mathrm{U} \cdot \mathrm{m}^{-2} \cdot \mathrm{s}^{-1}$. in $4 \pi$ steradians \\
\hline POWER OF FLUX OF UNIVERSONS & $\mathrm{P}=5.37 .10^{60}$ Watts $. \mathrm{m}^{-2} . \mathrm{sr}^{-1}$ \\
\hline CAPTURE CROSS SECTION OF UNIVERSONS & $\mathrm{S}=2.18 .10^{-31} \mathrm{~m}^{2} \cdot \mathrm{kg}^{-1}$ \\
\hline INTERACTION TIME OF AN UNIVERSON & $=7.8 .10^{-14}$ second \\
\hline \multicolumn{2}{|c|}{ WAVELENGTH OF UNIVERSON WAVE FUNCTION $\lambda(\psi)=\mathrm{Eu} / \mathrm{h}=\mathbf{2 . 3 3 8 6 . 1 0}-\mathbf{5} \mathbf{m}$} \\
\hline \multicolumn{2}{|c|}{ INTERACTION PHASE SHIFT OF WAVE FUNCTION $\Phi=\lambda(\psi)$ or $2 \pi$ radians } \\
\hline SOLID ANGLE OF NO RE-EMISSION & $\Omega=1.6 .10^{-21}$ steradians.$m^{-1} \cdot s^{2}$ \\
\hline ITATION TEMPERATURE OF THE I & $T^{\circ}=1.16 .10-8$ \\
\hline
\end{tabular}

\section{Prediction of Universe Expansion Effect on All Accelerations}

The Universe expansion effect is detailed into Appendix D. Our model predicts a supplementary cosmological acceleration added to any acceleration of matter into the Universe.

This very small value acceleration (about $7.10^{-10} \mathrm{~m} / \mathrm{s}^{2}$ ) is equal to the product of Hubble's constant $\mathrm{H}_{0}$ by the speed of light $\mathrm{c}$. This is a systematic phenomenon caused by the finite duration $\tau$ of the quantum interaction. This prediction has very strong consequences on all Gravitational accelerations into the Universe, solving enigmas.

\section{Confirmations of Our Models Predictions at Astronomical Scale}

Prediction of cosmological supplementary acceleration $H_{0 c}$ is confirmed by acceleration history of several interplanetary spacecraft. This is presented into Appendix $\mathbf{E}$.

Confirmation of the tiny action of this prediction $H_{0} c$ onto the Earth orbit is shown and also confirmed by a spacecraft into Appendix F.

Consequences of prediction $H_{0}$ acceleration onto stars orbits, into all galaxies, are confirmed by astronomical observations into Appendixes $\mathbf{J}$ and $\mathbf{K}$. That confirmation made obsolete Cold Dark Energy models of the enigmatic dynamics of matter into galaxies, and also CDM Cosmological models. The "MOND paradigm" is validated, except for $H_{0} c$ acceleration (Modified Newtonian Dynamics, Milgrom, 1983, 2008).

Effectively, from our model, and its confirmed predictions, no Dark Matter hypothesis is required.

\section{Confirmation of Our Models Predictions from Laboratory Experiments}

We already published (Poher, 2011), that strong accelerations of electrons into superconducting devices produce a predicted propelling effect from the emission of an anisotropic flux of gravitational quanta bearing a momentum.

And we also published (Poher \& Modanese 2017) that the emitted quantum flux by accelerated electrons pushes electrons into distant metallic conductors.

These two kinds of experiments, replicated elsewhere, are confirmations of our Inertia model. These experiments allowed us to confirm the parameters values of the model, by measuring the propelling effect and the distant induction of currents.

Another set of experiments, to be published soon, into which the Earth Gravity Acceleration is involved, confirm also the models predictions.

\section{Fundamental Physics Consequences}

Our Quantum models of Inertia and of Gravitation are surprisingly rich of many predictions and fundamental consequences. For example:

- Appendix A is not only a demonstration of the value $E u \tau$ of two of the models parameters. It is also a confirmation of the ideas that Louis de Broglie developed into Quantum Physics on the physical nature of the strange "Wave Function $\psi$ ". He considered that there was a real agitation of matter particles caused by a "hidden agitated milieu" he named "the thermostat" by analogy with thermodynamic baths.

This matter particles agitation is very real, we have modelled the physical process. This means that our 
models show the physical real nature of the wave Function of Quantum Physics, a really strong achievement.

- As well, Appendix $\mathbf{B}$, is not only an evaluation of the value of $E \mathrm{u}$, one of our models parameter. It is the proof of action of real quanta involved into Gravitational interaction; the enigmatic cause of free fall of cold Neutrons into the Grenoble Laue-Langevin Institute experiment performed in 2002 is therefore known.

- Appendix $\mathbf{C}$ is not only the calculation of several parameters values. It is also the observation of a real fact, the value of phase shift of the Wave Function of Universons, during an interaction with a matter particle: $\Phi=2 \pi$. So this is an indication of what is really such an interaction, not a "capture" nor an "absorption", a real phase shift. This is a really new phenomenon, and a very first opportunity to understand the true physical meaning of a Quantum Physics interaction.

- As well, Appendix G, about fluctuations, associated with Appendix D, about the prediction of the $H_{0} c$ supplementary Cosmological acceleration, are showing the resolution of a century long enigma about the "non Newtonian and non Einsteinian" behaviour of Gravitation at very large distances into galaxies.

Our model eliminates the need for any Dark Matter mass of matter to explain galaxies Astronomical enigmas, which is a strong result. Therefore new Cosmological models are to be imagined.

These are only examples, it is clear that our Quantum models offer much more to think about.

\section{Cosmological Consequences}

The Universe is fully governed by Gravitation. Astronomers are able to observe very faint luminosity galaxies with a very large redshift. These observations demonstrate that galaxies were assembled very early from stars soon after the Big Bang. And of course stars assembled themselves even earlier from matter and Gravitation.

So Gravitation was born during the big Bang. This means that the huge flux of Universons was created at the Big Bang instant, by an enigmatic process. After that instant there has apparently been not anymore creation of new Universons (there is no indication of an increase of $G$ value). Therefore, the actual value of the natural isotropic flux intensity we observe and calculate, from local observations of galaxies, was created at Big Bang instant, and then has expanded in all directions at the speed of light.

Therefore, immediately after the Big Bang, the density of the expanding "ball" of Universons was tremendous, all the actual number of these quanta were concentrated into a quite small sphere.

We have shown into Appendix H, expression H8, that the Gravitational constant value $G$ is proportional to the flux intensity $F u$. So, immediately after the big Bang instant, the Quantum Gravitational flux intensity and the Gravitational constant $G$ values were gigantic. Therefore the new created matter particles were submitted to Huge relativistic pushing effects. Their velocities were close to the velocity c of Universons because the interactions were more numerous into this very dense "soup" of pushing quanta. The flux was not yet isotropic. It was strongly expanding, pushing matter outwards.

Matter particles velocities, close to $c$, gave values of parameters $\beta=v / c$ and $\gamma=\left(1-v^{2} / c^{2}\right)^{-1 / 2}$ from relations (1) and (2) of our model respectively close to 1 and to 0 . These values changed completely the interaction conditions, more time duration (expression (7), more momenta exchanged, etc).

So this was not classical Inertia, but a kind of "very rapid inflation with a huge dilatation speed", precisely the inflation hypothesis which is proposed (with enigmatic process) into cosmological models. It justifies the abundance of Hydrogen, of Helium, of Lithium, into early gas clouds not yet assembled into stars.

A huge ball of free Universons was expanding at speed $\mathrm{c}$ in all directions after the Big Bang. It was free to expand, contrarily to the ball of photons, which were captured by hot charged elementary particles, unable to assemble into neutral atoms, during about 300000 years. So when the photons became suddenly free at atoms assembly, the outer frontier of the expanding ball of Universons was already 300000 light years away from the expanding ball of photons frontier.

The strong Quantum fluctuations into the early ball of Universons agitated strongly the new born matter particles, atoms and their emitted photons, giving the actually observed temperature fluctuations into the cosmological infrared radiation background.

Into that model of the Universe, the ball of Universons is permanently expanding since the Big Bang, it is locally observed to be isotropic at our scale, but it is not at Universe scale. The flux is anisotropic at very large scale, causing the acceleration of matter expansion at large scale, without any need for "DARK ENERGY". It is the Gravitational natural field, which is the cause of expansion acceleration observed from very distant supernovae explosions. 
Therefore the $\Lambda \mathrm{CDM}$ models of Cosmological evolution should be changed accordingly.

\section{Applied Physics Opportunities}

We undertook our laboratory work in 2006 to be able to verify our quantum model of inertia into a series of real experiments. From predictions of our theoretical models, we invented (and patented) specific devices made of sandwiches of superconducting materials and metallic conductors named "emitters" (Poher, 2011). These emitters were used to observe, with full success, the predicted specific physical facts that can now be exploited into industrial potential applications of great importance. They are mainly:

- The very strong "jet-like" propelling effects, from electric energy, without ejection of matter particles. The momentum of that propelling effect is proportional to the electric energy applied to our emitters. This property proves that there is energy extraction from the natural isotropic quantum field. This propulsion is quickly adaptable into most Spacecraft, because its energetic performance is already larger than any other electric propelling system. That propulsion method does effectively not require storage onboard of matter to be ejected in outer Space. The mass of spacecraft will be largely reduced, and their launch cost accordingly.

- The second major physical effect, the main one in our opinion, which is able to be used into industrial applications, is the induction of supplementary currents into distant conductors (Poher \& Modanese, 2017). Our emitters extract energy from the natural Gravitational flux. That effect can be used to produce static electric generators without any fossil resources use, and no polluting emissions. This became easier with invention of room temperature emitters in 2015. Electric cars generators are concerned, without recharge.

Other possible important applications, for later use, were imagined. The two main previous applications were studied into detail into our laboratory. They are actually ready for direct industrial R\&D work, with a relatively soon predicted availability of real systems.

Therefore it is time for industries prospection and exploitation licences of our patents, deposited for limiting international concurrence of concerned high technology industries.

\section{Conclusion}

A new kind of quantum physics models of Inertia and of Gravitation (twin brothers) is born. It is time now to forget Dark Matter hypotheses and improve and build new models.

The Natural isotropic flux of Gravitational Quanta is a proven reality. And it is really easy to explore it with low cost laboratory experiments made with superconducting material devices, as we have shown ourselves.

We named our hypothetic Gravitation Quanta "Universons", only because "Gravitons", from the standard model of interactions, were supposed to carry a negative momentum while ours carries really a positive one. This said, the previous name of "Graviton" for the real natural Quantum, could be used, with the indispensable condition of a change into its institutional definition.

We have proven that the natural flux of "Universons" carries a huge amount of kinetic energy, which is possible to use for humankind needs. And we have demonstrated experimentally how to extract a small part of that energy.

So we hope that others will undertake not only replications of our experimental work, but also its improvement as well as a much better understanding of our yet imperfect theoretical models.

A new era of applied physics is therefore born.

\section{Difficult Scientific Context and Institutional Environment}

Introducing a new model for such a fundamental interaction as Gravitation is a quite strong claim. So much famous researchers have failed into that domain. The general context has a strong influence on model acceptation. About Gravitation, the "intellectual hypothesis" of "Lambda Cold Dark Matter" was born onto another continent, almost simultaneously while we started ourselves to assemble the very first "stones-facts" of our Gravitation model. This was also simultaneous to the important work of Vera Rubin and her group, observing the enigmatic constant orbital velocities of stars into hundred of galaxies.

The $\Lambda$ CDM "ideas" originated from famous institutions. So it propagated quickly into all our own continent institutes, during the forty two years of our own "counter-current work", to build our own Gravitation quantum model. The environment was like a kind of religious worship, about costly and complex underground laboratories tracking "wimps" and similar "illusions" of hypothetic CDM quanta. This happened in spite of what were telling us billions of galaxies in the night sky, observed only by very few "night birds".

Moreover, we are Applied Physics researchers, our aim is real industrial applications. For this reason, we decided 
to assemble a maximum of confirmations of our models before publishing it. The unfavourable scientific environment, for our ideas, had to be compensated by a lot of predictions and of real observations. Our insistence about confirmations was necessary, because our work has been a "private only venture". Not any official institution was involved, even if our past professional activities were performed into several institutions.

\section{Acknowledgements}

We are sorry not being able to acknowledge any institutional organization from our country, of from Europe, for any help. Even we chose to publish into another friendly country, on the other side of ocean, because only Dark Matter models proposals were accepted on our side.

Our acknowledgements are therefore mainly for the members of our families and a few hidden friends for their long patience and consensus.

\section{Conflict of interests}

The authors declare that there is no conflict of interests regarding the publication of this paper.

\section{References}

Anderson, J. D., Laing, P. A., Lau, E. L., Nieto, M. M., \& Turyshev, S. G. (2001). The strange acceleration of Pioneer 10 and 11 . The planetary report (Planetary society, USA).

Anderson, J. D., Laing, P. A., Lau, E. L., Nieto, M. M., Turyshev, S. G. (2002). Study of the anomalous acceleration of Pioneer 10 and 11 . NASA Office of space science applications.

Bohm, D., \& Vigier, J. P. (1954). Phys. Rev., 96, 208.

CERN physics. Dark Matter. Retrieved from https://home.cern/science/physics/dark.matter

Collab. Planck, Plank 2018 results, VI, cosmological parameters.

De Broglie, L. (1925). Recherches sur la théorie des quanta, Paris, 1924 (thèse de physique). Ann. de Physique, 3(10), 22.

De Broglie, L. (1971). La réinterprétation de la mécanique ondulatoire. Gauthier-Villars Paris.

De Broglie, L. (1987). Interpretation of Quantum Mechanics by the theory of the Double Solution. Annales de la Fondation Louis de Broglie, 12(4).

Freedman et al. (2020). Calibration of the tip of the red giant branch. Astroph. J. to be published in 2020.

Heisenberg, W. (n. d.). The development of quantum mechanics. Nobel Lecture.

Milgrom, M. (1983). ApJ, 270, 365

Milgrom, M. (n. d.). «The MOND paradigm». arXiv :0801.3133 [astro-ph],.

NASA Science Astrophysics. Dark energy, Dark matter. Retrieved from https://science.nasa.gov/astrophysics/focus-areas/what-is-dark-matter

Nesvizhevsky et al. (2002). Nature, 415, 297-299.

Ostriker, J. P., \& Steinhardt, P. J. (1999). Cosmic Concordance. Retrieved from https://arxiv:astro-ph/9901388v2

Poher, C., \& Marquet, P. (2012). General Relativity and Universons. Applied Physics research, 4(1).

Poher, C., \& Modanese, G. (2017). Enhanced induction into distant coils by YBCO and silicon-graphte electrodes under large current pulses. Physics Essays, 30, 4.

Poher, C., \& Poher, D. (2011). Physical phenomena observed during strong electric discharges into layered Y123 superconducting devices at $77 \mathrm{~K}$. Applied Physics Research, 3(2), 51.

Riess et al. (2019). Large Magellanic Cloud Cepheid determination of the Hubble's constant. Astrophys. J., 882(1), 34.

Rubin, V., Thonnard, N., \& Ford, W. K. Jr. (1980). Rotational Properties of 21 Sc Galaxies with a Large Range of Luminosities and Radii from NGC $4605(\mathrm{R}=4 \mathrm{kpc})$ to UGC $2885(\mathrm{R}=122 \mathrm{kpc})$. The Astrophysical Journal, $238,471$.

Tully, R. B., \& Fisher, J. R. (1977). A New Method of Determining Distances to Galaxies. Astronomy and Astrophysics, 54(3), 661-673.

Wong et al. (202). Measurement of Ho from lensed quasars. MNRAS.

\section{Appendix A - Louis De Broglie'S Double Quantum Solution and Eu T Product Value}


A.1 - LOUIS DE BROGLIE, 1892 - 1987, was a brilliant mathematician, physicist, Nobel price 1929 winner after his 1927 thesis on the wavy behaviour of the electron.

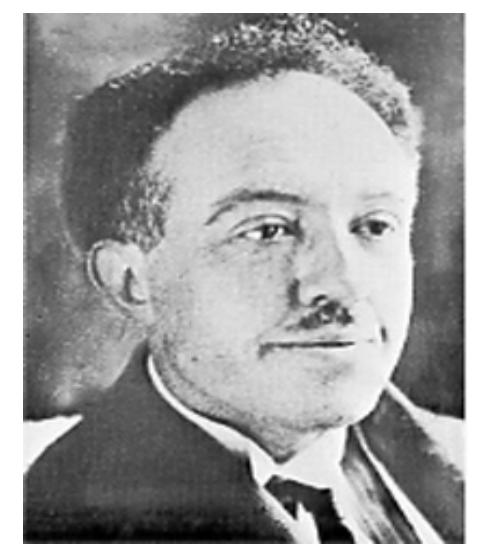

Figure A-1. Louis de Broglie — 1892 — 1987

Into Quantum Mechanics, it is not possible to determine the position and momentum of a matter particle with an infinite accuracy. Werner Heisenberg demonstrated that the product of these two parameters must always be larger than a fundamental parameter $h / 4 \pi$ from Max Plank's constant. $h=6.626 .10^{-34}$ Joule. Second.

The "Wave function $\psi$ " is the complex expression which defines the state of a particle. And Louis de Broglie demonstrated that a moving particle is "accompanied" by a wave, which represents the particle state at any instant (de Broglie, 1924, 1971, 1987). The wave function $\psi$ is defined by Schrödinger's fundamental equation.

This is a complex shape wave that can be considered made of an infinite sum of sinusoidal waves as demonstrated by Fourier.

The amplitude and phase of these components change from one place to another one, and the sum gives a maximum amplitude supposed showing where the particle should approximately be at a certain instant.

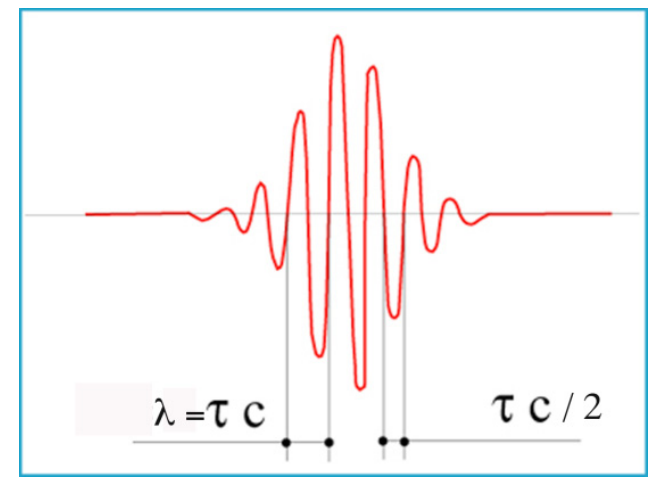

Figure A-2. Classical Representation of the wave function $\psi$ of one Universon, The wave amplitude varies along space and along time. It is never nil, in the past as well as into the future

The probability to find the particle at a certain place and for a certain time is known from the wave function $\psi$.

This probability is given by the square root of the sum of the squares of the complex and real components of $\psi$.

However, this way, the sum of probabilities is not equal to $100 \%$. A "Normalization" operation is required before the square root calculation. This is a multiplication by a constant given by an integral calculation.

Louis de Broglie was not satisfied by this normalization process. He considered that the operation was disturbing deeply our Quantum Mechanics understanding.

So he proposed to continue using the wave function $\psi$ and the normalization process, but with a "second solution". 
He supposed that the tiny oscillation amplitude of the calculated wave $\psi$ was the signature of a very real physical phenomenon associated with the moving particle interaction with an unknown sub quantum medium, having its own fluctuations, like it is the case into thermodynamics. He named "Thermostat" this hypothetical agitated fluid.

It is particularly fruitful nowadays to consider again L. de Broglie's hypothesis in the frame of the natural isotropic Universons flux model with its own fluctuations.

Effectively, the "thermodynamic" fluid proposed by L. de Broglie (his "thermostat") seems really to be the omnipresent bath of moving Universons, made of individual Quanta propagating at the velocity of light in all directions of space and interacting with matter particles.

Louis de Broglie suggested that his proposed "pilot wave" of matter particles was associated with the "Brownian" movement caused by the bathing quantum medium. Effectively, as we have shown, a matter particle is permanently agitated by the interactions with the Gravitational Universons. We even have calculated the "temperature" of that agitation $\left(1 \cdot 16 \cdot 10^{-8} \mathrm{~K}\right)$.

The L. de Broglie's idea was studied in 1954 by D. BOHM et J. P VIGIER with less details (Bohm, 1954).

\section{A.2 - Mass and Energy Fluctuations of Matter Particles (See Also Appendix G)}

Into our Universons model, we demonstrated that elementary matter particles with a mass $\mathrm{M}_{0}$ interact permanently with Universons. Each incident Universon increases the energy $M_{0} c^{2}$ of the matter particle by an amount $E u$, which is the kinetic energy carried by a single Universon. Consequently the instantaneous mass of a matter particle fluctuates permanently around the average value $M_{0} c^{2}$.

Evidently, the exact instants of Universons interactions with matter are never as perfectly synchronous as into figure A-3, because this is a random phenomenon, with a Gaussian statistical distribution around an average mass. This average mass value imposes that there are mass excursions up and down, meaning that there are as much incident Universons as emergent ones.

These mass steps are tiny, about $10^{-7}$ times the particle average mass for an electron as an example. And these mass fluctuations are quite fast, about 1.24 $.10^{18}$ times per second for an electron.

We can calculate that frequency. Let us call $N$ the average number of Universons in the interaction state with a matter particle of average mass $M o$.

We demonstrated that Newton's inertia Law : Force = Mass . Acceleration, gave us to the following result:

$$
N=M o c^{2} / E u
$$

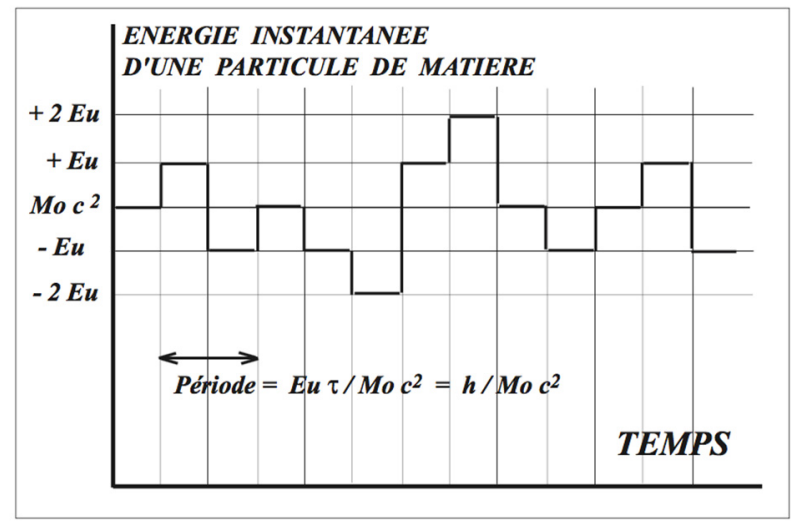

Figure A-3. Matter particle mass fluctuations caused by Universons interactions (pedagogical virtual Figure)

This is not a hypothesis, but a fundamental result, a consequence of our model. It means that the mass of elementary particles of matter is caused by the permanent interaction with Gravitational Quanta. For an electron, the number $N$ is about ten millions.

These $N$ Universons are permanently replaced. And this number fluctuates very rapidly, with a constant average value (Figure A-3). However the mass variations are "abrupt", equivalent to a rectangular oscillation of a peak energy amplitude $E u$ and a period equal to two times the interaction duration $\tau$. 
Each individual interaction duration is $\tau$ therefore ALL the $N$ Universons interacting simultaneously with the matter particle are entirely replaced after time $\tau$. So the frequency $F$ of the energy oscillations of the particle is:

$$
F=N / \tau=M_{o c} c^{2} / E u \tau
$$

This because for each interaction of an incident Universon, there is another one becoming free as it had interacted $\tau$ seconds earlier.

\section{A.3 - Louis De Broglie'S Double Quantum Solution Applied}

According to L. de Broglie, it is the preceding energy fluctuations that are the deep cause of the physical phenomenon of wave function $\psi$ of a matter particle. Effectively, we know that if the matter particle is accelerated, the Universons interaction is perturbed, and the incident and emergent Universons fluxes become anisotropic, so $\psi$ changes.

Moreover, if the matter particle velocity becomes relativistic, the interaction duration $\tau$ changes, it is increased, which modifies the frequency (A2).

Effectively the "little clock" postulated by L. de Broglie is remarkably present into figure A-3 where we have simply applied our Universons model.

And Louis de Broglie tells us the frequency beat of that little clock:

$$
v_{0}=M o c^{2} / h
$$

So with (A2) we can write (same frequencies):

$$
v_{0}=M o c^{2} / h=F==M o c^{2} / E u \tau
$$

From where comes immediately:

$$
E \boldsymbol{u} \tau=\boldsymbol{h}
$$

This result is fundamental. It is $4 \pi=12.57$ times larger than Heisenberg's quantum limit.

\section{A.4 - Conclusion of Appendix A}

We observe here that our Universons model reinforce the ideas expressed by L. de Broglie in 1929. We have here the beginning of understanding the deep physical nature of quantum phenomena.

By applying the results from L. de Broglie to our model, we confirm his ideas and mainly his intuition about the "little internal clock" he imagined. This justifies the wave function $\psi$ before the normalization process for the amplitudes of probability density.

The fact that oscillating fluctuations of positions and momentum of matter particles are always in phase with the wave function $\psi$ was proven by Louis de Broglie almost a century ago.

This early prediction has allowed us to tie narrowly the Planck's constant $h$ with the main parameters of our Inertia model, the gravitational quantum energy $E \mathrm{u}$, and its interaction duration $\tau$ with matter particles.

This is a result we consider to be very fundamental, because it gives a new meaning to Quantum Physics and to its numerous amazing results, which are a challenge to physicists.

\section{Appendix B - Proper Energy Eu of an Universon}

\section{B.1 - Nesvizhevsky'S and AL. Experiment}

The Universon energy can be deduced from results of the 2002 very difficult made experiment from Valery V. Nesvizhevsky \& al. into the Laue-Langevin Institute in Grenoble, France (Nesvizhevsky, 2002).

Into that experiment, ultra cold Neutrons are moving horizontally at low speed (of the order of 10 meters per second) in a narrow space between two horizontal and parallel planes along a 10 centimeters distance. The inferior plane is a mirror for the Neutrons, and the superior plane is an absorber for Neutrons.

The Neutrons moving slowly between the two plates are submitted to the gravity field from the Earth. They have parabolic trajectories in this narrow space, before going out, where they are detected and counted.

The experiment shows that no Neutron exits when distance between the two planes is smaller than 15 microns. Over this distance, the number of Neutrons increases rapidly with the distance : 8 times more at 20 microns than at 15 microns, 100 times more at 40 microns etc. 


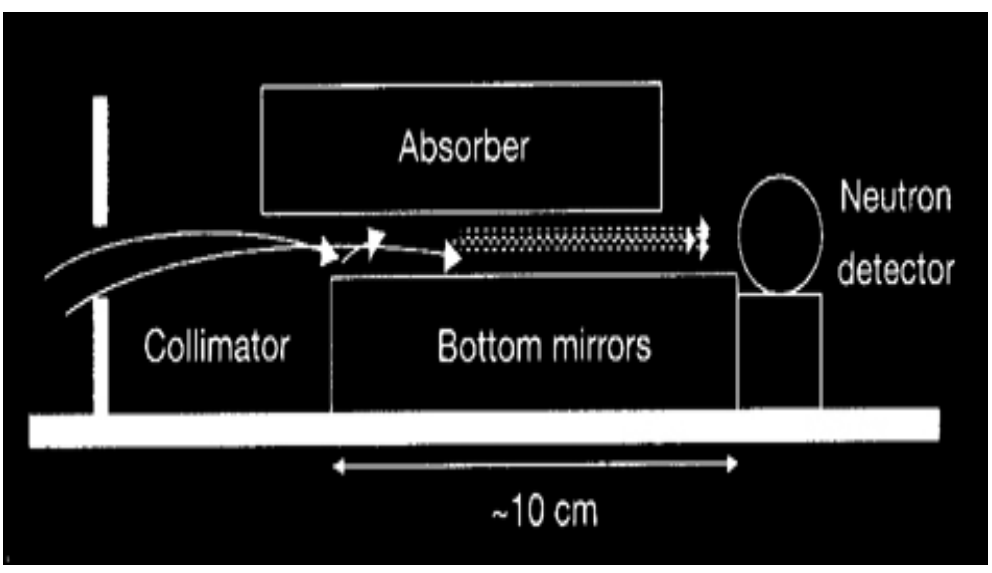

Figure B.1. Principle of Nesvizhevsky's experiment

\section{B.2 - Interpretation with Universons Model}

One can interpret this experiment in the frame of the Universons theory, considering that each Neutron is submitted to an anisotropic exchange of energy with the captured Universons, this gives the gravitational acceleration of the Neutrons and their parabolic trajectory. The energy that is needed to raise a Neutron by 15 microns in the Earth gravitational field is $1.5 .10^{-12}$ electron volt according to authors.

Effectively, this energy is given by the following classical physics relation:

$$
E=m g h=1.67 .10^{-27} \cdot 9.81 \cdot 15 \cdot 10^{-6}=2.4 \cdot 10^{-31} \mathrm{Joule}=1.5 \cdot 10^{-12} \mathrm{eV}
$$

As no Neutron goes out of the experiment when the distance between the two planes is less than 15 microns, one can consider that this is due to the fact that they are all absorbed. This tells us that they receive, during their travel along the 10 centimeters of the parallel plates, a larger kinetic energy than the one needed for a Neutron to be reflected by the inferior plate and absorbed by the superior one.

Then we can deduce that the minimum kinetic energy transferred to a Neutron, in the vertical direction, by the capture of Universons (corresponding to only one Universon) is $1.5 .10^{-12}$ electron volt.

Let us call $E u$ the kinetic energy transferred by an Universon to a Neutron during interaction. We know that the impulse communicated by the capture is equal to $E u / c$, where $c$ is the speed of light.

A Neutron of mass $M n$ gets, during capture a speed $v$ such as:

$$
M n v=E u / c
$$

Its kinetic energy is:

$$
E c=M n v^{2} / 2
$$

Then finally:

$$
E c=E u^{2} /\left(2 M n c^{2}\right)
$$

We know that:

$$
E c=1.5 \cdot 10^{-12} \mathrm{eV}
$$

Or:

$$
E c=2 \cdot 4 \cdot 10^{-31} \text { Joule }
$$

With $c=3.10^{8} \mathrm{~m} / \mathrm{s}$ and $\mathrm{Mn}=1 \cdot 67 \cdot 10^{-27} \mathrm{~kg}$, we get finally:

$$
E u=8.5 .10^{-21} \text { Joule }
$$

If the Universons energy was electromagnetic, this would correspond to a wavelength of:

$$
\mathrm{l}=h \mathrm{c} / \mathrm{Eu}
$$

Where $c$ is the speed of light and $h$ the Planck's constant. With $h=6,62 \cdot 10^{-34} . s$ we would get: 


$$
\mathrm{l}=2.34 \cdot 10^{-5} \mathrm{~m} \text { or } 23,4 \text { microns }
$$

This would be a very powerful infrared radiation easy to detect. As this radiation does not exist, we conclude that Universons don't bear an electromagnetic energy, only a kinetic energy.

Consequently, Universons don't make any difference between neutral (Neutrons) and charged (Protons, electrons) particles of matter, only their mass counts in their interaction.

\section{B.3 - Consequences for Universons Model Parameters}

In Universons model, capture and re-emission anisotropy of the Universons flux is expressed by two equal solid angles $\Omega$ given by:

$$
\Omega=2 \pi A \tau / c
$$

In this expression we use:

$-\Omega$ is the solid angle value (steradians)

$-A$ is acceleration of the matter particle $\left(\mathrm{m} . \mathrm{s}^{-2}\right)$.

$-\tau$ is the capture time of the Universons $\left(7.8 .10^{-14}\right.$ second).

$-c$ is the speed of light (299 $792458 \mathrm{~m} / \mathrm{s})$.

The average number $N$ of Universons, captured and re-emitted by the matter particle of rest mass $M$, during the capture time $\tau$ is equal to:

$$
N=M c^{2} / E u
$$

Expression where $E u=8.5 \cdot 10^{-21}$ Joule is the energy transferred by one single Universon to the particle when it is captured.

Moreover, a captured unidirectional flux of one Universon per second creates a force equal to (time $\mathrm{T}=1$ ):

$$
\text { Force }=E u / c \tau=2.83 .10^{-29} \text { Newton }
$$

\section{B.4 - What Happens During Acceleration of One Electron?}

The rest mass $M e$ of an electron is equal to:

$$
M e=9.11 \cdot 10^{-31} \mathrm{~kg}
$$

So, expression (B11) allows us to know the average number $\mathrm{Ne}$ of Universons captured by an electron:

$$
\mathrm{Ne}=9.6325 .10^{6} \text { Universons }
$$

These 9.6 millions Universons captured are re -emitted at the end of the capture time $\tau=7.8 .10^{-14}$ second, and this corresponds to a total re-emitted flux $\mathrm{Fr}$ equal to:

$$
F r=1.7263 .10^{20} \text { Universons per second in } 4 \pi \text { steradians }
$$

Consequently, the Universons flux $F i$ responsible of the inertia force, for an accelerated electron is:

$$
F i=2 \Omega F r / 4 \pi
$$

So, with (B11) and (B14):

$$
F i=A .3,21 \cdot 10^{-2} \text { Universons per second }
$$

Inversely, a captured unidirectional flux of one Universon per second accelerates the electron:

$$
\text { Acceleration of one electron for one Universon per second }=31.15 \mathrm{~m} . \mathrm{s}^{-2}
$$

\section{B.5 - Acceleration of a Proton or of a Neutron}

The rest mass $M p$ of a Proton is practically the same as the one of a Neutron, it is equal to:

$$
M p=1.67 .10^{-27} \mathrm{~kg}
$$

This is 1836 times the rest mass of an electron.

Therefore expression (B11) allows us to know the average number $N p$ of Universons captured by a Proton or by a Neutron: 


$$
N p=1.7685 .10^{10} \text { Universons }
$$

Consequently, the Universons flux Fpi responsible of the inertia force, for an accelerated Proton or Neutron is:

$$
F p i=3672 \Omega \mathrm{Fr} / 4 \pi
$$

So, with (B11) and (B20):

$$
F p i=A .58 .9 \text { Universons per second }
$$

Inversely, a captured unidirectional flux of one Universon per second accelerates the Proton or the Neutron:

$$
\text { Acceleration of a Neutron for one Universon per second }=0.017 \mathrm{~m} . \mathrm{s}^{-2}
$$

\section{B.6 - Discussion of Nesvizhevsky's Experiment Interpretation Validity}

We have determined the energy transferred to an elementary particle of matter (a Neutron) by a captured Universon, thanks to the results of the experiment from Valery V. Nesvizhevsky \& al.

Into this experiment, ultra cold Neutrons are moving horizontally at low speed (of the order of 10 meters per second) in a narrow space between two horizontal and parallel planes along a 10 centimeters distance. Consequently, the Neutrons stay between the two plates of the experimental apparatus during about 0.01 second.

According to previous expression (B22), the flux Fpi of the Universons responsible for the Inertia force acting on one accelerated Neutron is 58.9 Universons per second per $\mathrm{m} . \mathrm{s}^{-2}$ acceleration.

In the Grenoble experiment, the acceleration $A$ applied to the Neutrons is the Gravity acceleration of the Earth in Grenoble:

$$
A=9.81 m \cdot s^{-2}
$$

So, during 0.01 second of the Neutrons travel time between the two plates of the experiment, the average number Npi of acting Universons during the «free fall» of the Neutrons in the local gravitational field is equal to:

$$
N p i=9.8 .58 .9 .0,01=5.8 \text { Universons per Neutron on average }
$$

This number Npi fluctuates during time, from one Neutron to the next, according to a statistical distribution described by Laplace and Gauss, with a standard deviation equal to $N p i^{1 / 2}$ :

$$
\text { Standard deviation of the fluctuations of Npi }=2.4 \text { Universons }
$$

Strictly speaking, the number Npi being smaller than 18 , we should be at the border of validity of the Laplace-Gauss' distribution, and we should use instead the Poisson's distribution. But the «validity zone» is quite «fuzzy» between these two distributions and it appears that changing the distribution type would not change the nature of the result.

So the real number of Universons to consider, to determine their action on the Neutrons in the experiment, is between 1 and 10 for $95 \%$ of the Neutrons, because the real number is the average one \pm 2 times the standard deviation. This is the definition of the statistical distribution we are using.

Each Neutron reacts to the exchange of a linear momentum with 1 to 10 Universons, for $95 \%$ of the Neutrons entering the experiment slit.

This means that, at this particle scale, the real fall speed of each Neutron can vary by a factor ten for $95 \%$ of them, but the statistical average fall velocity should be the one corresponding to 5.8 Universons. The fall speed must be clearly quantified, because the linear momentum can only vary by steps of one Universon capture momentum, that is : $\mathrm{Eu} / \mathrm{c}=2.83 \cdot 10^{-29} \mathrm{~kg} \mathrm{~m} / \mathrm{s}$.

The experiment is precisely conceived as a filter, that allows to pass only Neutrons which fall speed is lower than a precise threshold, and we can interpret this threshold as corresponding to an interaction of the Neutron with one or zero Universon during its transit time of 0.01 second.

So, it appears, with real numbers introduced in the expressions, that this experiment can be interpreted this way, taking into account the interaction fluctuations of the Universons by a Neutron.

These considerations do comfort the value of parameter $E u$, from experimental results, and they comfort also the values of the other parameters of the theory.

Appendix C - Definition Relations and Parameters Values in Universons Model

\section{C.1 - Notations Used in Fundamental Relations Are the Following}


$A=$ Acceleration of matter. $\left(\mathrm{m} \cdot \mathrm{s}^{-2}\right)$.

$\tau=$ Capture time of an Universon in a particle of matter. (Seconds, s).

$\Omega=$ Solid angle where there is no re-emission of the captured Universons. (Steradians, sr).

$\mathrm{F}=$ Cosmological, isotropic flux of free Universons. (Universons . $\mathrm{m}^{-2} \cdot \mathrm{s}^{-1}$. in $4 \pi \mathrm{sr}$ )

$\mathrm{S}=$ Capture cross section of Universons by matter. $\left(\mathrm{m}^{2} \cdot \mathrm{kg}^{-1}\right)$.

$\mathrm{Eu}=$ Proper energy of an Universon. (Joules).

$\mathrm{c}=$ Speed of light. $\left(\mathrm{m} . \mathrm{s}^{-l}\right)$. We use here $\mathrm{c}=3.10^{8}$ instead of $2.99792458 .10^{8}$.

$\mathrm{G}=$ Universal Gravitation Constant. (Newton. $\mathrm{m}^{2} \cdot \mathrm{kg}^{-2}$ ). We use here $\mathrm{G}=6.67 .10^{-11}$

$\mathrm{H}_{0}=$ Hubble's constant (We use here $\mathrm{H}_{0}=75 \mathrm{~km} / \mathrm{s}$ per mega parsec).

$\mathrm{h}=$ Planck's constant. $\mathrm{h}=6,626 \cdot 10^{-34}$ joule . second.

$\lambda=$ Wavelength of the Universon wave function (meters).

$\Phi=$ Phase shift of an Universon wave function during interaction with a matter particle (radians).

\section{C.2 - Fundamental Relations from Our Model}

Several of these relations are detailed into other appendixes.

$$
\begin{gathered}
\Omega=2 \pi \mathrm{A} \tau / \mathrm{c} \\
\tau=\mathrm{c}^{2} /(\mathrm{F} \mathrm{S} \mathrm{Eu}) \\
\mathrm{G}=\mathrm{Eu} \mathrm{F} \mathrm{S}^{2} /(4 \pi \mathrm{c})=\mathrm{S} \mathrm{c} / 4 \pi \tau \\
\tau \quad / S=3.58 .10^{17} \\
\mathrm{Eu} \tau=\mathrm{h} \\
\mathrm{F} \tau \quad=3.8 .10^{54} \\
\lambda(\psi)=\mathrm{Eu} / \mathrm{h} \\
\Phi(\psi)=\tau \mathrm{c}
\end{gathered}
$$

\section{C.3 - Parameters Values}

REST ENERGY OF AN UNIVERSON

ISOTROPIC NATURAL FLUX OF UNIVERSONS

FLUX OF UNIVERSONS POWER

CAPTURE CROSS SECTION OF UNIVERSONS

INTERACTION TIME OF AN UNIVERSON

WAVELENTH OF UNIVERSION WAVE FUNCTION

INTERACTION PHASE SHIFT OF WAVE FUNCTION

RE-EMISSION SOLID ANGLE OF UNIVERSONS

AGITATION TEMPERATURE OF INTERACTION

COSMOLOGICAL EXPANSION ACCELERATION

$$
\mathrm{Eu}=8.5 .10^{-21} \mathrm{Joule}
$$

$$
\mathrm{F}=6.3 .10^{80} \mathrm{U} \cdot \mathrm{m}^{-2} \cdot \mathrm{s}^{-1} \text {. in } 4 \pi \mathrm{sr}
$$

$$
\mathrm{P}=5.37 .10^{60} \text { watts } . \mathrm{m}^{-2} . \mathrm{sr}^{-1}
$$

$$
\begin{aligned}
& \mathrm{S}=2.18 .10^{-31} \mathrm{~m}^{2} \cdot \mathrm{kg}^{-1} \\
& \tau \quad=7.8 .10^{-14} \text { second } \\
& \lambda(\psi)=2.3386 .10-5 \mathrm{~m} \\
& \Phi=\lambda(\psi) \text { or } 2 \pi \text { radians } \\
& \Omega=1.6 .10^{-21} \text { steradians } . \mathrm{m}^{-1} \cdot \mathrm{s}^{2} \\
& \mathrm{~T}^{\circ}=1.16 .10^{-8} \text { Kelvin } \\
& \mathrm{H}_{0} \mathrm{c}=7.1 .10^{-10} \mathrm{~m} / \mathrm{sec}^{2} \pm 5 \%
\end{aligned}
$$

\section{C.4 - How Were Obtained These Relations and Fundamental Parameters Values ?}

Relation (A) corresponds to the laws of energy and momentum conservation, and to the experimental fact that the rest mass of a matter particle remains constant on average.

Relation (B) is the translation of Newton's law determining the Inertia force for accelerated matter, in the Universons model.

The consequence of this fundamental relation is that the proper mass of all the elementary particles of matter must be made of the total energy of all the simultaneously captured Universons, which are permanently replaced in the particles. Relations (B), (C), (D), and (F) are detailed in Appendix H. 
Relations $(\mathrm{C})$ are the result of the value of the anisotropic flux of Universons exchanged by two masses of matter, caused by their gravitational acceleration.

Relation (D) is coming from (B) and (C).

Relation (E) demonstration comes from Appendix A.

Relation (F) was obtained from (B), (C) and (D).

The value of the energy $E u$ comes from Appendix B.

Relations (G) and (H) come from quantum physics (L. de Broglie's theory).

Values of the fundamental parameters have been calculated from $E u$ by using the fundamental relations presented here.

\section{Appendix D - Effect of Universe Expansion}

\section{D.1 - Introduction}

We demonstrate that Universe expansion increases the acceleration of any accelerated particle of matter, by a constant acceleration, equal to $\mathbf{H}_{\mathbf{0}} \mathbf{c}$. With $\mathbf{H}_{\mathbf{0}}$ being the Hubble's constant, and $\mathbf{c}$ the speed of light.

\section{D.2 - After - Interaction Anisotropy of Universons Trajectories}

During the interaction time $\tau$ of one Universon, when the matter particle is accelerated by an external cause (whatever it may be), with an acceleration $\mathbf{A}$ there is an increase of its velocity by a quantity $\mathbf{V} \mathbf{1}$ equal to:

$$
\mathbf{V} 1=\mathbf{A} \tau
$$

Therefore the re-emitted Universon, with speed c cannot simultaneously bring with it the energy it had when interacting, and adopt also a departure direction identical to the one it had when captured.

We know, experimentally, that elementary particles of matter of the same type have all the same mass (even if we don't know theoretically why). This imposes to the re-emitted Universon to take back with it the exact energy it had when captured.

This description of real facts is quite simplified. A relativistic theory of the interaction shows that it is necessary to avoid considering the individual interactions of Universons with particles of matter, because many Universons interact simultaneously. At least it is necessary, for avoiding errors of interpretation, to consider Universons interactions by pairs, with opposed incident trajectories. However, this very simplified description gives correct results, and it has the advantage to be very simple to understand.

A re-emitted Universon, in a direction making an angle $(\phi)$ with direction $\mathbf{x}$ of the acceleration $\mathbf{A}$, has, in all cases, a speed $\mathbf{c}$ relative to the matter particle. The component $\mathbf{V} \mathbf{x}$ of the Universon velocity, in the direction $\mathbf{x}$ of the matter particle acceleration is then equal to:

$$
V x=\mathbf{c} \cos (\phi)
$$

The re-emitted Universon must adopt a minimum change in its own direction of departure $(\phi)$ which is such that $\mathbf{V x}=\mathbf{c}-\mathbf{V 1}$ and it is from these considerations that the anisotropy of re-emission of the Universons have been explained on a simplified way. But this can be demonstrated more rigorously by more exact methods.

\section{D.3 - Matter Velocity Due to Universe Expansion}

Let us consider now a NOT captured Universon. It is supposed traveling along axis $\mathbf{x}$ of the matter particle acceleration, with speed c. We suppose that this Universon goes through the matter particle without being captured, but we suppose also that it is close enough to another Universon that is captured. Now we look at the behaviour of the two distinct Universons.

During interaction time $\tau$, of the second Universon, the first one travels the distance $\mathbf{D} 1$ along axis $\mathbf{x}$ :

$$
\mathbf{D 1}=\mathbf{c} \tau
$$

And, during that same time $\tau$, the Universe expands, so distance D1 expands and becomes D2:

$$
\text { D2 }=\text { D1 }\left(1+\tau \mathbf{H}_{0}\right)
$$

Expression where $\mathbf{H}_{\mathbf{0}}$ is the well-known Hubble's constant defining the Universe expansion rate. Previous Expression (D4) is simply the definition of the Hubble's constant $\mathbf{H}_{0}$.

So, distance D1 has increased by a quantity $\mathbf{D} 1 \tau \mathbf{H}_{\mathbf{0}}$ during time $\tau$. 
A distance traveled during a certain time is a velocity. So Universe expansion is equivalent to velocity $\mathbf{V 2}$ :

$$
\mathbf{V} 2=(\mathbf{D} 2-\mathrm{D} 1) / \tau
$$

Replacing terms by their values:

$$
\mathbf{V} \mathbf{2}=\mathbf{c} \tau \mathbf{H}_{0}
$$

So, for this NOT - captured Universon, everything is just like if there was no Universe expansion, but a supplementary speed of the matter particle $\mathbf{V} \mathbf{2}$ along axis $\mathbf{x}$. However the matter particle is accelerated. And its speed increases already by $\mathbf{V} \mathbf{1}$ along this same axis $\mathbf{x}$. So, the Universe expansion, for an accelerated particle of matter, is equivalent to an increase of its speed, during the capture time $\tau$, by the sum $\mathbf{V}$ :

$$
\mathbf{V}=\mathbf{V} 1+\mathbf{V} \mathbf{2}
$$

Or:

$$
\mathbf{V}=\mathbf{A} \tau+\mathbf{c} \tau \mathbf{H}_{\mathbf{0}}=\tau\left(\mathbf{A}+\mathbf{H}_{\mathbf{0}} \mathbf{c}\right)
$$

The re-emitted Universon must now adopts a new minimum direction change $(\phi)$ such that $\mathbf{V x}=\mathbf{c}-\mathbf{V}$ and it is from these considerations that the re-emission anisotropy of the Universons have been explained on a very simplified way, with the Universe expansion included.

\section{D.4 - Supplementary Acceleration $\mathrm{H}_{0} \mathrm{c}$}

It suffices now to compare relations (D1) and (D8) to see that the Universe expansion introduces a supplementary acceleration of the matter particle equal to $\mathbf{H}_{\mathbf{0}} \mathbf{c}$. The new acceleration $\mathbf{A}^{\prime}$ of matter particle is now equal to:

$$
\mathbf{A}^{\prime}=\mathbf{A}+\mathbf{H}_{\mathbf{0}} \mathbf{c}
$$

In this expression, $A$ is the matter particle own acceleration (whatever its cause) and Hoc is a constant acceleration, very tinny (about $7.1 .10^{-10} \mathrm{~m} / \mathrm{s}^{2}$ ), added by Universe expansion.

Hubble's constant $\mathbf{H}_{0}$ is known from different methods, giving close but not exactly same values (Anderson, 2001, 2002). Five methods, considering the actual Universe expansion, give an average value of $73.06 \mathrm{~km} / \mathrm{s}$ per Mpc [69.6 From red giant stars (Freedman, 2020), 73.3 from Holicow Quasar (Wong, 2020), 73.6 from Mira stars, 74.0 from Cepheid stars (Riess, 2019), 74.8 from Megamasers]. And three other methods, considering an older Universe, give an average value of $67.606 \mathrm{~km} / \mathrm{s}$ per Mpc [67.4 from Plank's anisotropies of background light (Plank Collab, 2018), 67.4 from baryons oscillations, and 68.0 from gravitational waves from the past]. Therefore that cosmological acceleration value depends actually on the source of $\mathbf{H}_{\mathbf{0}}$ value.

There is here a very fundamental aspect: if interaction duration $\tau$ of Universons was nil (did not exist) then $\mathbf{V} \mathbf{1}$ and $\mathbf{V} \mathbf{2}$ would be nil, and there would be no constant acceleration $\mathbf{H}_{\mathbf{0}} \mathbf{c}$ added.

The fact that acceleration $\mathrm{H}_{0} \mathrm{c}$ has been REALLY observed, thanks to Pioneer 10, Pioneer 11, Galileo, and Ulysses spacecraft, proves two fundamental facts:

\section{Gravitational interaction is REALLY OUANTIFIED.}

Interaction time duration $\tau$ exists REALLY.

\section{Appendix E - Universons Model Confirmations by Trajectories of Interplanetary Spacecraft}

\section{E.1 - Introduction and Principles}

Into Appendix $\mathrm{D}$ we demonstrated a prediction, by the Universons model, of the existence of a constant, cosmological, acceleration $H_{0} c$.

When matter is accelerated at a level larger than $H_{0} c$, we will show into Appendix $\mathbf{K}$ that such conditions exist into the Solar system, where the Sun gravitational acceleration is larger than $H_{0} c$ up to a distance of more than 2855 Astronomical Units is reached.

Here for confirmation of our model, we use of the trajectory of free, distant, interplanetary, spacecraft because the perturbations of their acceleration by internal propulsion are nil in cruise phase. The idea behind this choice is the following: the constant acceleration $H_{0} c$ should not change with the distance of spacecraft from the Sun, while the gravitational acceleration does.

This fact could allow, in principle, to distinguish the two effects and put the constant acceleration into evidence. 
Effectively, the gravitational acceleration into the Solar system is much larger than the constant acceleration caused by the expansion of the Universe $H_{0} c$ for short distances. And the most distant explored planet (Pluto, $39.44 \mathrm{AU}$ ) is closer than the $2855 \mathrm{AU}$ limit where the gravitational acceleration from the Sun is equal to $H_{0} \mathrm{c}$.

Space professionals have the real need to know accurately the acceleration of spacecraft. Such are the space engineers in charge of the space navigation of interplanetary spacecraft. They use complex software to adjust automatically the navigation errors observed, and decide the trajectory modifications to be made occasionally by the propulsion systems of the spacecraft.

However, the needs in trajectory prediction accuracy for interplanetary exploration spacecraft increase with the performances of these missions and their cost. Low trajectory prediction accuracy means more propulsion mass ejected in Space, embarked onboard, to insure the success of the mission, and consequently less scientific payload and more cost. Scientific demands in this domain are more and more ambitious, in order to understand the Astronomical objects of the Solar system, better known since the beginning of the Space era.

This increasing demand explains the progress made by the trajectory prediction of remote spacecraft since the beginning of Space exploration, about sixty years ago. Trajectories are measured with a very high accuracy, thanks to the Doppler effect on radio waves exchanged by the Earth ground stations and the spacecraft. All spacecraft are equipped with transponders for this purpose, but the frequency accuracy $\left(10^{-15}\right)$ is installed into ground stations.

Measurement of the Doppler effect is always one of the scientific objectives of these spacecraft missions, because it is a mean to determine the gravitational field properties of the destination planets. This measure is also used for the verification of Einstein's General Relativity theory with an increased accuracy, and also for the knowledge of the characteristics of the interplanetary medium and of the Solar wind.

The few rare specialists of these questions are actually able to determine acceleration perturbations of interplanetary spacecraft with an accuracy of the order of $\pm 10^{-10} \mathrm{~m} . \mathrm{s}^{-2}$ at a distance of $80 \mathrm{AU}$ from the Sun.

This is exactly what is needed for the confirmation of the Universons model prediction.

Nevertheless we cannot use any spacecraft trajectory for our purpose, because spacecraft are experiencing many acceleration perturbations, larger than the one we would like to confirm.

This means that it is essential to be able to measure effectively all perturbations, in order to extract the previously unknown, constant, acceleration $H_{0} c$ we have predicted.

What are the accelerations acting on a spacecraft in free space flight?

- There is the gravitational acceleration from the Sun, which decreases as the square of the distance of the spacecraft from the Sun.

- There is also the gravitational acceleration from planets and their satellites, which decreases as the square of the distance of the spacecraft from each planet. These change constantly.

- There is an acceleration caused by Solar photons radiation pressure, decreasing also with the square of the distance, but subject to variations with solar activity. It varies also slowly with the modification of characteristics of radiation absorption and reflection of the spacecraft materials due to the space environment, because the mission duration is generally quite long (years, even decades).

- There is an acceleration caused by the pressure of the solar wind made of particles, narrowly connected with the variable solar activity.

The determination of all these accelerations uses complex computer software, and a detailed and accurate knowledge base, of the solar system, and of the permanent solar activity, improved by the ground and space research results.

- Another not negligible acceleration is the one caused by thermal radiation of the spacecraft itself. This acceleration does not change with distance, but with the electric consumption and generation of the spacecraft systems. For interplanetary spacecraft, this acceleration is dominated by the thermal radiation of the radio-isotopic electric generators, because the use of solar panels is impossible so far from the Sun.

- Finally, there are numerous accelerations caused by the spacecraft systems themselves, such as the propulsion manoeuvres for trajectory correction, the eventual gas jets impulses of the attitude control system (orientation by rotation of the spacecraft for the control of its antennas or experiments aiming in Space).

- There appear also undesired micro leaks of the pressurized systems (propulsion as well as attitude control). They generally decrease during a few days after a propulsive voluntary action. 
- There is also accelerations caused by the radiation pressure of the few watts of radio waves, produced by the spacecraft radio transmitters, concentrated generally by parabolic antennas towards the Earth, moving in Space.

All these accelerations must be modelled, carefully followed, and recognised by their temporal or distance variation, by a regular measurement of the trajectory of spacecraft, deduced from the radio Doppler effect. And sometimes a Doppler plus range radio measurement, from modulated radio waves.

\section{E.2 - What Are the Perturbations That Modify the Accuracy in the Determination of Spacecraft Accelerations from Ground Based Stations?}

There are modifications of the influence of the interplanetary medium, the influence of density of the solar corona, the inaccuracy of time synchronisation between ground stations. At least three ground stations are necessary around the globe, because Earth rotates, when the spacecraft distance is so large that the round trip time of radio waves is larger than the visibility duration of a spacecraft from one station. For example, the round trip time of radio waves for Pioneer 10 was larger than 21 hours in 2002.

We have recalled here these perturbations in order to show the seriousness of the specialists of interplanetary spacecraft. These measurements are done with help from the exceptional instruments of NASA deep space network (DSN). Spacecraft trajectory studies are made by very experienced specialists. They have a real professional need for accurate results, to insure the success of costly space missions.

Space trajectories specialists have developed unique methods, radio electric and software powerful tools, to attain the best possible accuracies in spacecraft accelerations determination.

They are generally not known by the Public, nor by physicists, but their skills and results merit a warm recognition by the scientific community.

\section{E.3 - Spacecraft to Consider}

The use of trajectories of terrestrial spacecraft must be excluded for our verification, because the perturbations in Earth orbit are much larger than the effect we want to see.

For example the random variations of acceleration, caused by aerodynamic braking from the near Earth residual atmosphere, would mask completely acceleration $H_{0} c$.

There are also regional irregularities of the gravitational potential of Earth, which would mask also the effect to determine.

Consequently, to be able to measure an acceleration of the order of $7.10^{-10} \mathrm{~m} / \mathrm{s}^{2}$ with minimum error, we must choose spacecraft in free flight, as far as possible from the gravitational and residual atmospheric perturbations of solar system planets, and from the Sun. The list of spacecraft corresponding to these trajectory constraints is quite short. They are old interplanetary spacecraft, having used, in their past, the gravitational pull of heavy planets, such as Jupiter or Saturn, to travel now far from them, towards the outside of the solar system. These spacecraft are the only ones far from the largest gravitational perturbations.

The existing spacecraft with a trajectory corresponding to our needs are the following:

\section{Pioneer 10, Pioneer 11, Voyager 1, Voyager 2.}

Nevertheless, the Galileo, Ulysses, Near and Cassini spacecraft have also interesting trajectories, but to a lesser degree.

Moreover, the selected spacecraft for our purpose, must also have strict characteristics concerning the acceleration perturbations produced by their internal systems:

- The spacecraft must not have active propulsion systems, able to produce random or unknown accelerations.

This is mainly the case when a three axis attitude control system uses gas jets for the orientation of the spacecraft. For this reason we must choose spin stabilized spacecraft, which use no (or rarely, at voluntary moments) gas jets, for maintaining their orientation in Space. Unfortunately, this constraint eliminates the two Voyagers, but all other stay in our list.

- The spacecraft must be equipped with a radio transponder, compatible with trajectory equipment of the DSN, and this transponder must be functioning normally, and be powerful enough to be received by the DSN.

- Finally it must be possible, for the chosen spacecraft, to determine and follow the evolution of all other known acceleration perturbations, such as solar radiation pressure, radiation pressure from the thermal emission of the spacecraft, and its electrical generators, etc ... This implies knowledge and the active control of orientation of the axes of the spacecraft in Space, relative to an absolute frame of reference, without unknown perturbations. 
So there remain only three spacecraft of our list satisfying all the constraints: the two Pioneers and Galileo. The Ulysses spacecraft seems interesting, but it has very frequent attitude control manoeuvres that perturb its trajectory by the unknown associated micro leaks.

Precisely, NASA has observed, since many years, for all these four spacecraft, a constant, "unexplained" acceleration, towards the Sun. This acceleration modifies the trajectories of these spacecraft in a way incompatible with the Laws of Kepler and Newton.

Constant acceleration $H_{0} c$ has been first observed on Pioneer 10, as soon as 1980, when the spacecraft reached about 20 AU from the Sun. Since that date, more spacecraft have revealed the same "apparent anomaly".

\section{E.4 - Constant Acceleration of Pioneer 10}

The scientific mission of Pioneer 10, launched March 2nd 1972, was terminated with success a long time ago $(31 / 3 / 1997)$.

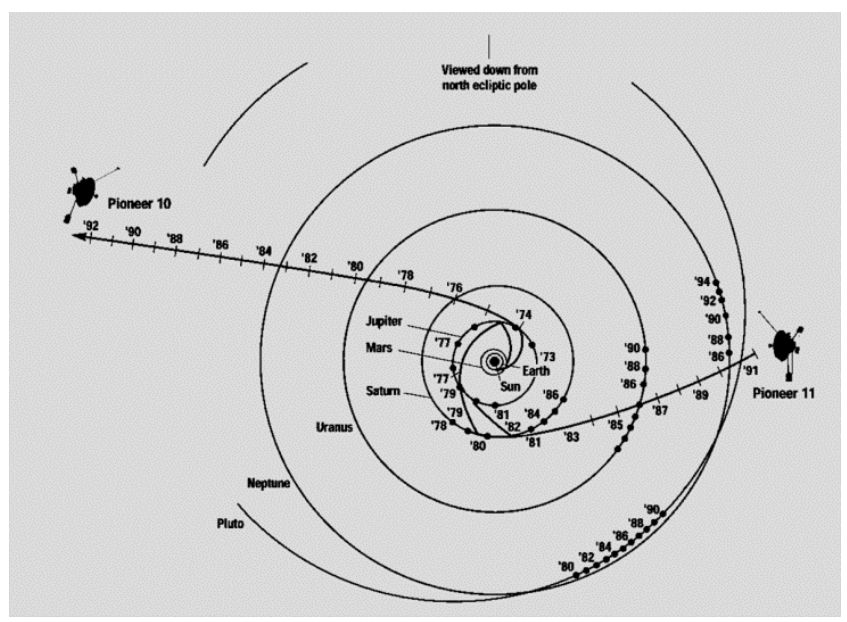

Figure E-1. Trajectories of the Pioneers into the solar system

This spacecraft was tracked by NASA, Deep Space Network antennas, as a difficult target, until February 2003. Since that date it has no longer been able to be contacted.

Pioneer 10 was, at the end of 2002, at a distance of about $80 \mathrm{AU}$ (Astronomical Units - One $\mathrm{AU}=1.5 .10^{8} \mathrm{~km}$ ), this is still a zone where the solar gravitational acceleration is much larger than $H_{0} c$.

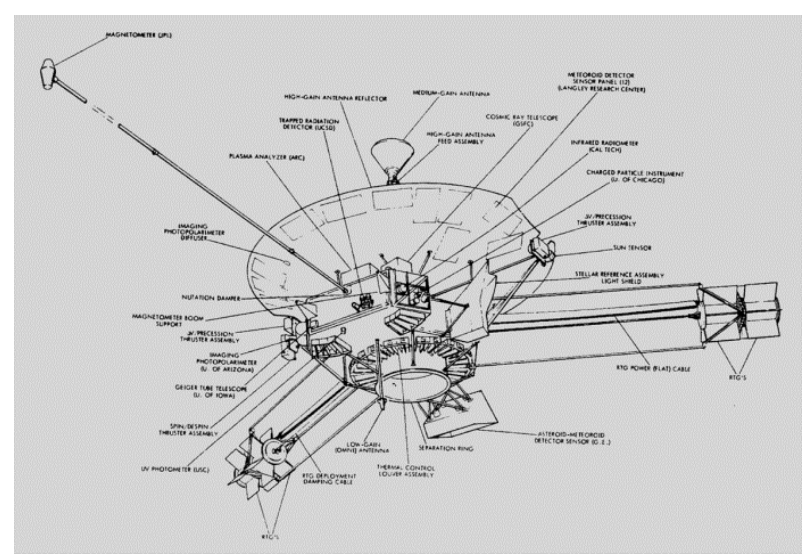

Figure E-2. Pioneer 10 \& 11 were stabilized by rotation, their antenna oriented towards Earth

Anderson (2001, 2002), NASA - JPL scientist in charge of the Doppler experimentation of Pioneer 10, published the discovery of an "anomalous" constant acceleration of Pioneer 10 towards the Sun, existing since its discovery in 1980, and still present in 2002. This acceleration had an amplitude of $8.7410^{-10} \mathrm{~m} / \mathrm{sec}^{2}$ (The published accuracy was $\pm 15 \%)$. 
The Pioneer 10 spacecraft is travelling away from the Sun, about in the plane of the ecliptic, in the general direction of star Aldebaran.

During year 1980, Pioneer 10 was 20 AU from the Sun, so NASA supposed first that its acceleration was caused by Solar radiation pressure. But this radiation acceleration was at this time lower than $5.10^{-10} \mathrm{~m} / \mathrm{s}^{2}$. For this reason, the previous constant acceleration became evident and "anomalous" (Markward, 2007).

\section{But for us, this experimental result confirms clearly the prediction of our Universons model.}

\section{E.5 - Constant Acceleration of Pioneer 11}

Pioneer 11 spacecraft, launched in April 1973, finished its mission since a long time (1990). Its transponder failed in October 1990, at a distance of 30 AU from the Sun.

The same constant acceleration has been measured from the $10 \mathrm{AU}$ of useful trajectory of Pioneer 11 . This constant acceleration is oriented towards the Sun, its published value is $8.56 \cdot 10^{-10} \mathrm{~m} / \mathrm{sec}^{2}$. (The published accuracy is also $\pm 15 \%$ ). (Anderson, $2001 \& 2002$, Turyshev, 2005).

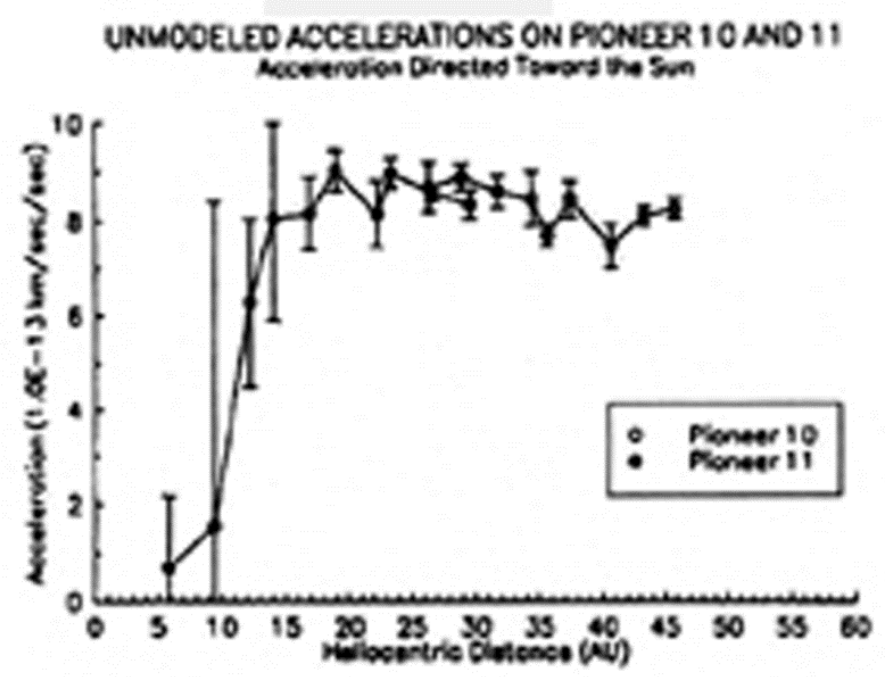

Figure E-3. Constant accelerations data of Pioneer 10 and Pioneer 11. There was still a residual thermal radiation acceleration from 20 to $35 \mathrm{AU}$ distance.

Toth \& al. (2007) suggested to explain the anomalous constant acceleration of the Pioneers spacecraft by the infrared radiation pressure from the RTG power generators installed at end of a long boom, photons bouncing back onto the communications antenna. This kind of thermal radiation propulsion seems effectively to be a small part of the total observed acceleration in Figure E-3, as this one is slowly decreasing with time. And this suggestion would explain why the real acceleration value is larger than $H_{0} c\left(8.74 .10^{-10}\right.$ instead of $\left.7.1 .10^{-10} \mathrm{~m} / \mathrm{s}^{2}\right)$, suggesting that the real thermal radiation push was of the order of only $1.64 .10^{-10} \mathrm{~m} / \mathrm{s}^{2}$ and not the full value observed.

\section{E.6 - Constant Acceleration of Galileo Spacecraft}

Galileo spacecraft is a little less adequate for our confirmation purpose, because this spacecraft has been maintained in the planet Jupiter environment, 5.2 AU from the Sun, where the gravitational perturbations are quite large. Fortunately the Galileo spacecraft had also a long and quiet trajectory from Earth to Jupiter, where the measures we needed could be performed.

Moreover, this spacecraft had a new transponder able to give both its Doppler speed as well as its distance to the Earth. For Galileo, NASA has also observed a constant acceleration towards the Sun: $8.0 .10^{-10} \mathrm{~m} / \mathrm{s}^{2}$ (With a published accuracy of $\pm 38 \%$ ).

\section{E.7 - Constant Acceleration of Ulysses Spacecraft}


The Ulysses space mission was not well fitted to our needs, because this spacecraft had an elliptical orbit too close to the Sun (about $5 \mathrm{AU}$ ), inclined $80^{\circ}$ from the ecliptic plane. However, there were two Doppler transponders into this spacecraft, using different frequencies, and also a distance measurement system. This allowed a determination of low accelerations closer to the Sun than it was possible for Pioneer 10 and 11.

However Ulysses spacecraft, stabilized by spin, was re-oriented periodically quite often, because its antenna had to stay oriented towards the Earth, a constraint caused by its peculiar orbit around the Sun.

This re-orientation used gas jets, produced gas leaks, and long periods of time with acceleration perturbations.

Nevertheless NASA has found for Ulysses, a constant acceleration towards the Sun, its published value was:

$12.10^{-10} \mathrm{~m} / \mathrm{s}^{2}$. (Published accuracy is $\pm 3.10^{-10} \mathrm{~m} / \mathrm{s}^{2}$ )

NASA specialists estimated that the Ulysses measurements of that constant acceleration was not very significant because they had great difficulty to determine the acceleration perturbations caused by the orientation gas jets manoeuvres imposed by scientific mission of the spacecraft.

\section{E.8 - Other Interplanetary Spacecraft}

The Pioneers had no active propulsion for attitude control, contrarily to the Voyagers, and to New Horizons. There is also Cassini that stopped at Saturn. A constant acceleration of the order of Pioneer 10 one, during 47 years of space cruise, without any thrusting, corresponds to a velocity change of $1.3 \mathrm{~m} / \mathrm{s}$ only. A difficult task indeed. Velocities of those interplanetary spacecraft are actually the following (Vitesse in French):

\begin{tabular}{|c|c|c|c|c|c|}
\hline & Pioneer 10 & Pioneer 11 & Voyager 2 & Voyager 1 & New Horizons \\
\hline Distance au Soleil (UA) & 125,800 & 104,274 & 123,536 & 148,927 & 47,034 \\
\hline Vitesse relative au Soleil $(\mathrm{km} / \mathrm{s})$ & 11,931 & 11,220 & 15,323 & 16,965 & 13,956 \\
\hline Vitesse relative au Soleil (UA/an) & 2,517 & 2,367 & 3,232 & 3,579 & 2,944 \\
\hline Latitude écliptique & $3^{\circ}$ & $14^{\circ}$ & $-37^{\circ}$ & $35^{\circ}$ & $2^{\circ}$ \\
\hline Déclinaison : & $25^{\circ} 55^{\prime}$ & $-8^{\circ} 46^{\prime}$ & $-58^{\circ} 13^{\prime}$ & $12^{\circ} 19^{\prime}$ & $-20^{\circ} 17^{\prime}$ \\
\hline Ascension droite : & $5^{\mathrm{h}} 9^{\mathrm{m}}$ & $18^{\mathrm{h}} 54^{\mathrm{m}}$ & $20^{\mathrm{h}} 5^{\mathrm{m}}$ & $17^{\mathrm{h}} 15^{\mathrm{m}}$ & $19^{\mathrm{h}} 16^{\mathrm{m}}$ \\
\hline Constellation & Taureau & Écu de Sobieski & Paon & Ophiuchus & Sagittaire \\
\hline Distance à la Terre (UA) & 126,372 & 104,100 & 123,482 & 148,431 & 46,922 \\
\hline Distance-lumière (heures) & 17,52 & 14,43 & 17,12 & 20,57 & 6,50 \\
\hline Luminosité du Soleil depuis la sonde (Magnitude) & $-16,2$ & $-16,6$ & $-16,2$ & $-15,8$ & $-18,3$ \\
\hline Sonde active? & non & non & oui & oui & oui \\
\hline Date de lancement & 1972-mars-03 & 1973-avr.-06 & 1977-août-20 & 1977-sept.-05 & 2006-janv.-19 \\
\hline
\end{tabular}

Figure E-4. Trajectories characteristics of five interplanetary spacecraft in 2020

From figure E-4 data, we see that measuring a velocity change of $1.3 \mathrm{~m} / \mathrm{s}$ from a $11 \mathrm{~km} / \mathrm{s}$ velocity represents an accuracy of the order of $0.01 \%$ for the Pioneers and should be about $0.007 \%$ for the Voyagers. Therefore it was really an achievement from JPL specialists to be able to pinpoint the Pioneer anomalous acceleration. With spacecraft having active propulsion for attitude control, this is really almost impossible to measure.

\section{E.9- Appendix E Conclusion}

There are acceptable agreements, despite technical difficulties, between experimental acceleration measurements made from several spacecraft and the prediction from the Universons model:

Pioneer $10=8.74 \pm 1.33 .10-10 \mathrm{~m} / \mathrm{s}^{2}$. Possible RTG thermal radiation tiny residual acceleration added.

Pioneer $11=7.44$ to $9.84 .10^{-10} \mathrm{~m} / \mathrm{s}^{2}$. (Accuracy $\pm 15 \%$ ). Same RTG remark.

Galileo $=5.79$ to $11.10-10 \mathrm{~m} / \mathrm{s}^{2}$ (Accuracy $\left.\pm 38 \%\right)$.

Ulysses $=9.6$ to $15.10^{-10} \mathrm{~m} / \mathrm{s}^{2}$. (Accuracy $\pm 25 \%$ Questionable because many attitude manoeuvres)

Universons model $=6.58$ to $7.1 .10^{-10} \mathrm{~m} / \mathrm{s}^{2}$. (Accuracy $=$ the one of Ho measurement $( \pm 5 \%)$ ).

\section{Appendix F - Universons Model Prediction of Earth Orbit Modification}

\section{F.1 - Supplementary Acceleration $\mathrm{H}_{0} \mathrm{c}$}

Universons model predicts, for all accelerated masses, the existence of a supplementary acceleration $H_{0} c$ to the main acceleration of any mass. This is caused by the expansion of the Universe and interaction time duration.

This is an universal effect, existing for any acceleration, whatever its cause.

The supplementary acceleration $H_{0} c$ adds always to the primary one, as its direction is exactly the same. The value of the supplementary acceleration is constant, for the gravitational accelerations existing in the solar system. 
By " acceleration ", we mean " any kind of acceleration ". It can be a rocket push, a gravitational pull, and, of course, a change in the direction of the trajectory of the body, like the "centrifugal" acceleration we feel in a car during a curve. This kind of $H_{0} c$ effect should exist for all planets.

However, planets trajectories in the solar system are not the same as the one of the Pioneers spacecraft, which are approximately moving straight, in the opposed direction of the Sun.

All planets have orbits in the shape of an ellipse, the Sun occupying one of the two focuses of the ellipse. But these orbits are almost close to a circle. The "eccentricity" of the orbit expresses its axes change as compared to a circle radius. For six of the nine planets, the orbit eccentricity is less than $6 \%$. For planet Mars orbit, it is $9.3 \%$, and only Mercury and Pluto have orbits with an eccentricity larger than $20 \%$.

Let us consider the modifications of orbital equations predicted by our Universons model.

\section{F.2 - Modifications of Circular Orbits}

While this is not the case for the planetary orbits in the solar system planets, circular orbits are simple to understand. Let us suppose that a single planet is orbiting a star, and let us call:

— Ms the star mass,

- Mp the planet mass,

$-\mathrm{R}$ the circular orbit radius,

$-\mathrm{G}$ the gravitational constant $\left(\mathrm{G}=6.67 \cdot 10^{-11}\right)$,

- Ag the gravitational acceleration of the planet,

- Vp the orbital velocity of the planet,

- $\mathrm{P}$ the orbital period, or one turn duration.

The gravitational acceleration $\mathrm{Ag}$ of the planet, considered in a reference frame the origin of which is fixed at the star center, is given by Newton's law:

$$
A g=G(M s+M p) / R^{2}
$$

Now, the Universons theory says that there must be a supplementary acceleration $H_{0} c$ added to the gravitational acceleration. So the value of the acceleration acting really on the planet is:

$$
A g=H_{0} c+G(M s+M p) / R^{2}
$$

The planet has a circular orbit and a speed $V p$ along this orbit, such that the "centrifugal" acceleration compensates exactly the value of $A g$ given by relation (F2). This is expressed by:

$$
V^{2} / R=A g
$$

Or:

$$
V^{2} / R=H_{0} c+G(M s+M p) / R^{2}
$$

So the orbital period $\mathrm{P}$ is simply the orbit circle length divided by the orbital speed:

$$
P=2 \pi R / V
$$

And, evidently:

$$
V=2 \pi R / P
$$

Let us use the value of the orbital speed, given by expression (F6) into expression (F4):

$$
4 \pi^{2} R / P^{2}=H_{0} c+G(M s+M p) / R^{2}
$$

\section{F.3 - Modification of Kepler'S Third Law}

Expression (F7) can be written:

$$
\boldsymbol{R}^{3} / \boldsymbol{P}^{2}=\boldsymbol{H c} \boldsymbol{R}^{2} / 4 \pi^{2}+G(M s+M p) / 4 \pi^{2}
$$

This is a modification of Kepler's third law for orbits of planets. Effectively Kepler's law is:

$$
R^{3} / P^{2}=G(M s+M p) / 4 \pi^{2}
$$

\section{So, there is an added term:}




$$
H_{0} c R^{2} 4 \pi^{2}
$$

The effect of this added term is equivalent to a supplementary mass $\mathbf{M}^{*}$ of the star, equal to:

$$
M^{*}=H_{0} c R^{2} / G
$$

For a Sun mass, and a planet orbiting at one astronomical unit distance (the Earth), the apparent mass increase of the star would be quite small: $1.182 .10^{-7}$ of the star mass.

But it is clear that, for a galaxy, where the orbital radius of external stars is very large, the apparent galaxy mass would be very large too. This fact explains the existence of hypothetic "Dark Matter", as it will be shown in the next pages.

In any case expression (F8) says that both the radius and the period of the circular orbit are modified as compared with the Kepler's law.

For planets of the solar system, where it is easy to measure the exact period of orbits from the Earth, by simply looking at the position of the planets in the sky, the orbital radius of a circular orbit is changed, as compared with the one calculated from the Kepler's third law.

Let us call $R k$ the keplerian radius of the circular orbit. One calculates that the real radius $R u$ of the orbit, predicted by the Universons theory, is given by expression:

$$
R \boldsymbol{u}=\boldsymbol{R k}+H_{0} c P^{2} / 12 \pi^{2}
$$

So this is a quite small effect for the solar system planets.

But, in a galaxy, where the period of the orbiting stars is quite large, of the order of hundred of millions of years, the orbital radius change is significant.

\section{F.4 - Modifications of Elliptical Orbits}

We must consider the details of the effect of the Universe expansion on the particles of the planet matter.

In the solar system, the gravitational acceleration of a planet is the result of an anisotropy of the incident Universons flux, coming from the acceleration of the Sun mass.

In the Sun, there is a constant acceleration $H_{0} c$, added to the acceleration of the particles of the Sun, and this increases, a little bit, the anisotropy of the Universons reaching a planet.

So, the gravitational acceleration, which is acting on the planet, is a little larger than the Newtonian one, because there is $H_{0} c$ added to it. This added $H_{0} c$ acceleration is oriented towards the Sun, exactly as it is the case for Pioneer 10.

The augmented gravitational acceleration moves the particles of the planet towards the Sun. Their free trajectory is changed, this is exactly like if a « centrifugal acceleration » existed for each particle of the planet matter.

In the planet matter, there is also an effect caused by the Universe expansion, because Universons remain interacting with a particle of matter during a non-nil time.

So, the « centrifugal acceleration » is increased by a constant acceleration $H_{0} \mathrm{c}$.

This second $H_{0} c$ effect is not at all oriented towards the Sun, it is oriented in the same direction as the «centrifugal acceleration». This means it is perpendicular to the local trajectory of the planet, and oriented approximately in the opposite direction of the Sun.

So, finally, the Universons model predicts the existence of TWO supplementary accelerations $H_{0} c$ acting on a planet. The first one, created in the Sun, is oriented towards the Sun. The second one, created in the planet, is oriented perpendicularly to the orbit trajectory, towards the outside.

\section{For a circular orbit, the two accelerations are exactly opposed and they cancel each other.}

Nevertheless, the force, acting on the particles of the planet mass, is increased by the solar $H_{0} c$ acceleration, and the orbital radius of the circular orbit is changed as mentioned previously.

For an elliptical orbit, the two $H_{0} c$ accelerations are not aligned, and they do not cancel each other, except when the planet is positioned at two particular points of the orbit: at the perihelion and at the aphelion, where the planet is respectively at its closest and at its farthest distance from the Sun.

The angle between the two $H_{0} c$ accelerations varies slightly around its $2 \pi$ average value along an orbital turn. As a consequence, the resultant, $A r$ of the two accelerations, is oriented in the direction of the orbital speed, and 
varies as a sinusoid versus time $\mathrm{t}$, along the orbital period. Its amplitude is equal to product of $H_{0} c$ by the eccentricity $e$ of the orbit:

$$
A r=e H_{0} c \sin u t
$$

with,

$$
u=2 \pi / P
$$

So, for the Earth:

$$
u=2 \pi /(365.25 .24 .3600)
$$

The orbit is no more a perfect ellipse. There are tinny deformations that can be evaluated with the use of a simple computer simulation.

The computer simulation of the Earth orbit, by the authors, as well as for elliptical orbits of the other solar system planets, shows that their orbits remain stable when modified as indicated by the Universons model. The parameters of the Universons model orbits are evidently changed as compared with Keplerian orbits. But the modifications are so tiny that they are not actually observed from the Earth surface. Effectively, for all planets, the Newtonian gravitational acceleration of the Sun, and the gravitational perturbations of the other planets, are considerably larger than the Ar acceleration. For the Earth orbit, the computer simulation shows that the semi major axis of the ellipse is increased by 6425 meters, about the Earth diameter.

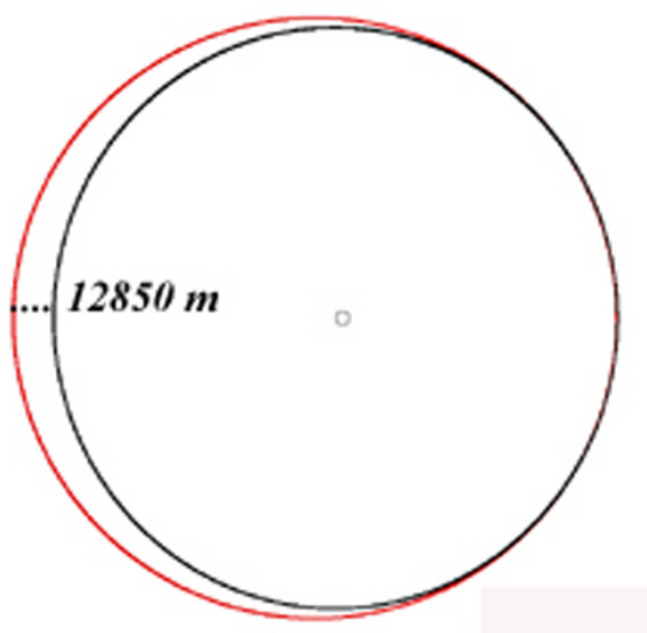

Figure F-1. Modification of the semi major axis of Earth orbit by H0 c acceleration

\section{F.5 - Earth Orbit Seen from Pioneer 10 Interplanetary Spacecraft}

From a computer simulation, we have shown, figure F-1, the difference between two kinds of orbits of the Earth, the one obtained with the classical Newtonian method, and the other one obtained with the Universons model.

However in order to see the difference on paper, we must exaggerate by a factor 100000 the value of $H_{0} c$.

Figure F-1 is the result we obtained with this exaggeration.

In this figure, the external, red Earth orbit is the one predicted by the Universons model, and the black one is the classical orbit defined by Newton's law. The small circle near the center is the Sun, and, for clarity, the two perihelion are the same.

Now, let us consider these two orbits as seen from Pioneer 10. The spacecraft is approximately situated in the plane of the figure, but very far, about 80 times the Sun-Earth distance, and to the top of the figure.

From Pioneer 10 position, let us measure the difference $\Delta \mathrm{d}$ of the distances of the Earth to the spacecraft from the two orbits, while each simulated Earth moves along its own orbit.

\section{The spacecraft sees a periodic distance difference $\Delta d$ with a period of one year.}


There are two opposed maxima per turn, the distance difference $\Delta d$ varying between plus and minus 6425 meters, with two zeros per year. The evolution of $\Delta \mathrm{d}$ versus time is modelled by (fundamental wave only):

$$
\Delta d=6425 \sin u t
$$

With $u$ given by (F15).

This means that the three "Deep Space Network" radio stations, situated on the Earth, do not measure the same accelerations of the Pioneer spacecraft, if we consider one orbit or the other, for the Earth.

Precisely, NASA-JPL does not use the Universons model for its computer models for the Earth orbit, as well as for the orbits of all other bodies in the solar system, to determine the acceleration of Pioneer 10.

The two consequences of this fact are the following:

- If the Universons model is correct, then, the model actually used by NASA-JPL is incorrect, and in this case, Pioneer 10 should have recorded the periodic variation of $\Delta \mathrm{d}$ given by expression (F16).

- If the Universons model is incorrect, there must be no periodic variations of the distance of the spacecraft to the Earth.

Expression (F16) does not shows the periodic acceleration Ap caused by the periodic variation of the distance between the two theoretical orbits. But we can calculate this acceleration by the derivative of (9-16) two times, because, by definition, acceleration is the second derivative of the distance. We get:

$$
A p=6425 u^{2} \sin u t
$$

With, for the Earth:

$$
\mathrm{u}=2 \pi /(365.25 .24 .3600)=1.991 .10^{-7}
$$

The value of Ap is then:

$$
A p=2.547 \cdot 10^{-10} \sin u t
$$

This is a periodic acceleration, of one year period, with a peak amplitude of $2.547 .10^{-10} \mathrm{~m} / \mathrm{s}^{2}$. It has two maxima and two zeros per year.

The peak to peak value of this periodic acceleration App is two times the amplitude $A p$ :

$$
\text { App }=5.09 \cdot 10^{-10} \mathrm{~m} / \mathrm{s}^{2}
$$

This is the theoretical value. It has the accuracy of the $H_{0} c$ value (about $\pm 5 \%$ ).

Now, let us consider what has been really measured by NASA-JPL, thanks to the Pioneer 10 spacecraft.

We need to know the value of the Pioneer 10 acceleration with a sufficient time resolution, in order to see the annual, sinusoidal, variation predicted. This has been published in April 2002 (Anderson \& al. 2002).

\section{F.6 - Real Pioneer 10 Spacecraft Acceleration Data}

High-resolution acceleration-residuals, measured by NASA-JPL, have been published, for the period 1987 - 1998 by Dr. ANDERSON and his colleagues. These are the remaining accelerations, after extracting from the data the constant $H_{0} c$ acceleration, as well as all other modelled accelerations. Figure F-2 is the data. 


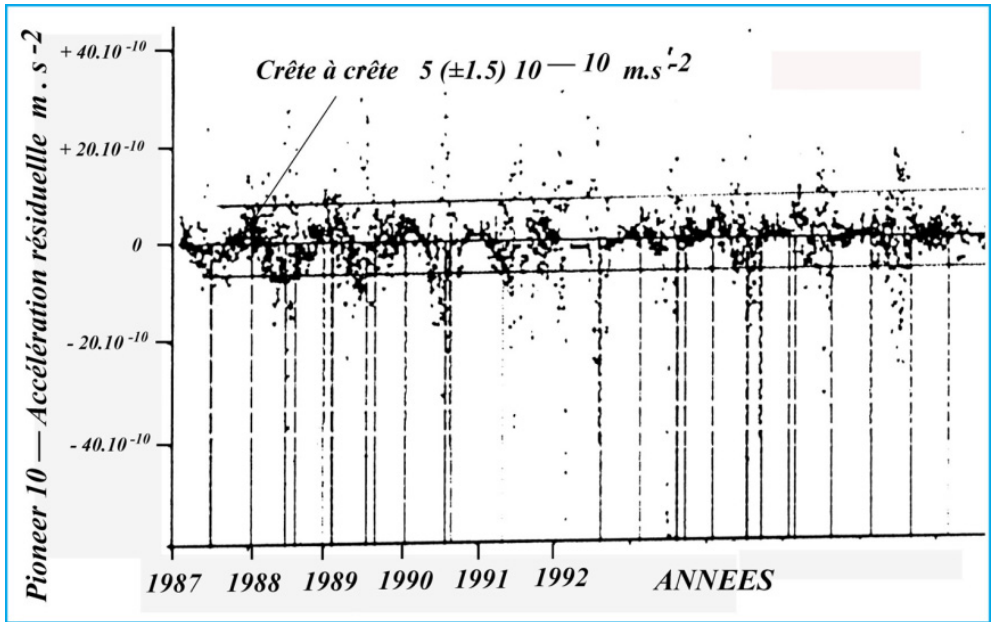

Figure F-2. Earth acceleration seen by Pioneer 10 trajectory

There is clearly a periodic observed acceleration, particularly between 1987 and 1992, because, during this period, the signal was less noisy, as the spacecraft was closer to the Earth.

The periodic acceleration has a one year period, and a peak to peak value of:

$$
\text { App observed }=5( \pm 1.5) .10^{-10} \mathrm{~m} / \mathrm{s}^{2}
$$

If we compare (F19) and (F20), we conclude that the theoretical value of the periodic acceleration predicted by the Universons model is exactly the one observed, thanks to Pioneer 10 and NASA-JPL.

\section{This confirms the predictions of the Universons model.}

The data presented by NASA-JPL in figure F-2 is quite "noisy". This is the result of a low signal to noise ratio.

Effectively, the 8 watts transmitter of Pioneer 10 was more than 40 astronomical units from the Earth at this time. Nevertheless, it has been possible to extract the relative acceleration of Pioneer 10 in these conditions. This has to be appreciated as a major challenge, amplified by the kind of technology used on board Pioneer 10, the one of the seventies.

Such a periodic acceleration could not be explained by an hypothetic thermal radiation acceleration from a RTG electric generator onboard Pioneer 10 as it has been proposed by an author for the constant pioneer 10 acceleration anomaly. Therefore that is a REAL effect, not an artefact.

\section{Appendix G - Quantum Fluctuations of Inertia Interactions}

\section{G.1 - Random Fluctuations of Natural Isotropic Flux of Universons}

We demonstrated that Inertia is the consequence of existence of the natural isotropic flux of Universons. This flux is composed of a huge density of individual similar quanta propagating at the speed of light in all directions of space. This flux is fluctuating randomly. This means that the intensity of this flux is not strictly constant everywhere and at each instant. There are natural variations of the flux intensity around an average value.

These natural fluctuations are equivalent to the superposition of an isotropic flux, strictly constant, to a random anisotropic flux varying in intensity and direction. The natural isotropic flux is the object of natural, random, fluctuations.

All natural phenomena made of individual particles, such as photons, elementary matter particles, rain, etc, are fluctuating randomly around an average value, and they are modelled by a mathematical model studied by Laplace and Gauss.

\section{G.2 - Laplace-Gauss Statistical Distribution of Fluctuations}

All phenomena following this natural statistical distribution vary randomly around an average value. They are generally made of very numerous individual quanta.

Let us call $x$ the random variable, and $f(x)$ the statistical distribution (probability) of the natural phenomenon around an average value $m$ of the $x$ variable. The mathematical law defining the Laplace-Gauss distribution uses 
another parameter named «Standard Deviation $\sigma »$. The relation between $x, f(x), m$, and the standard deviation $\sigma$ is the following:

$$
f(x)=\frac{1}{\sigma \sqrt{2 \pi}} e^{-\frac{(x-m)^{2}}{2 \sigma^{2}}}
$$

So, to any value of the variable $x$ corresponds a value of the probability $f(x)$ that changes the following way in function of $x$ around the average value $m$ :

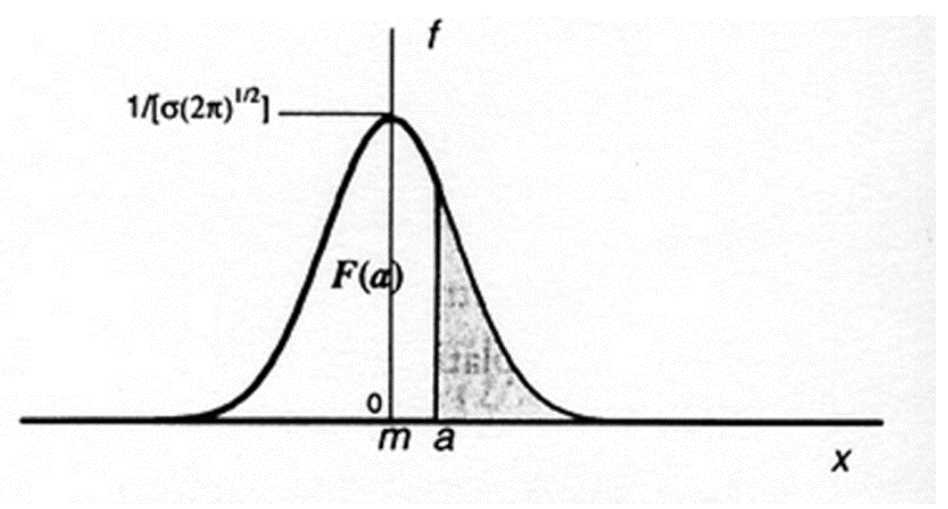

Figure G-1. Standard distribution of a quantity $\mathrm{x}$ fluctuating randomly around an average value $\mathrm{m}$

We observe that the probability $f(x)$ is maximum for the value $x=m$ and that it decreases before and after. Let us consider that $x$ represents the number of Universons going through a particle of matter each second (This is the incident flux on the particle). And let us suppose that $f(x)$ is the probability to observe the $x$ value of the incident flux at any time. This flux should be measured in Universons per square meter, per second, coming from all the directions of space.

Statistical Laplace-Gauss' distribution law says that the Universons flux is not constant. It varies around the average value $x=m$.

So, statistical Laplace-Gauss' distribution says also that the Universons flux is randomly distributed along time.

During $60 \%$ of the time, it has a value between the two limits: $x=m \pm \sigma$.

During $95 \%$ of the time, it has a value between the two limits: $x=m \pm 2 \sigma$.

And during $99.8 \%$ of the time, it has a value between the two limits: $x=m \pm 3 \sigma$.

In figure G1, we have represented a particular value of the flux $x=a$, corresponding to $x=m+\sigma$. The «Gauss curve» says that this value of the flux is less probable than the one corresponding to $x=m$.

In probability calculus, is sometimes used also another value called «Variance». It is simply the square of the standard deviation $\sigma$. So the standard deviation of $x$ is also defined as: $\operatorname{Var}(x)=\sigma^{2}$.

\section{G.3 Fluctuations into the Interactions of Universons}

Our Universons model says that any elementary particle of matter, having a non-nil rest mass, captures and re-emits permanently Universons from the natural, cosmological, flux. The captured Universons are systematically re-emitted after a certain «capture time» $\tau$ that several experimental facts demonstrate constant.

This theory has demonstrated also that the rest mass of an elementary particle of matter is made of the $N$ Universons in captured state simultaneously during time $\tau$, and permanently renewed.

We named $E u$ the energy transferred to a particle of matter at rest by a captured Universon, and momentum $P u$ of the Universon, before its capture, was (refer to Universons model presentation into main text):

$$
P u=E u / c
$$

Where $c$ is the speed of light (this is also the velocity of free Universons). This momentum is integrally transferred to the matter particle during the interaction phenomenon. Consequently, if $M$ is the rest mass of the matter particle, a capture of any Universon travelling along axis $\mathrm{x}$ communicates a constant velocity $V x$ to the particle, such as:

$$
P u=M V x
$$


So we get:

$$
V x=E u / M c
$$

This constant speed moves the matter particle along the $x$ axis during the capture time $\tau$ of the Universon, on a total distance $d x$ given by:

$$
d x=V x \tau=E u \tau / M c
$$

Each captured Universon is therefore changed into a very small fraction $m$ of the total mass $M$ of the particle, following the relation:

$$
m=E u / c^{2}
$$

Evidently, the average number Nmoy of captured Universons, simultaneously kept interacting with the matter particle is equal to:

$$
\text { Nmoy }=M / m=M c^{2} / E u
$$

The natural Universons flux being quantified, there are quantum fluctuations of the flux. This means that the real number $N$ of Universons simultaneously captured during time $\tau$ manifests random variations, around the average value Nmoy.

The distribution of the real values $N$ of the simultaneously captured Universons follows, along time, a statistical Laplace-Gauss distribution, characterized by two values:

- On the one hand, an average value Nmoy previously determined,

- And, on the other hand, a standard deviation $\sigma_{\mathrm{n}}$ that indicates the deviations around the average value.

According to Laplace-Gauss statistics law, we know that, during $99.8 \%$ of the time, the real number $N$ of Universons in simultaneous captured status with the matter particle varies between two limits: Nmoy $\pm 3 \sigma_{n}$.

We know also that, during $95 \%$ of the time, the real number $N$ of Universons in simultaneous captured status with the matter particle varies between two limits: Nmoy $\pm 2 \sigma_{n}$.

And finally, during $60 \%$ of the time, the real number $N$ of Universons in simultaneous captured status with the matter particle varies between two limits: Nmoy $\pm \sigma_{n}$.

Evidently, we cannot predict, at a precise instant, the exact value of the number $N$ because it varies randomly.

However, we can know the value of the standard deviation $\sigma_{\mathrm{n}}$ of the number of Universons in simultaneous captured status:

$$
\sigma_{\mathrm{n}}=\left(\text { Nmoy. } 2^{-1 / 2}\right)^{1 / 2}
$$

Where the square root of 2 takes care of the average quadratic deviation. According to (G6):

$$
\sigma_{\mathrm{n}}=\left(M c^{2} \cdot 2^{-1 / 2 / E u}\right)^{1 / 2}
$$

\section{G.4 - Fluctuations of Matter Particles Rest Mass}

The «instantaneous» rest mass $M i$ of the matter particle is proportional to $N$ and given by:

$$
M i=N E u / c^{2}
$$

Consequently, as $N$ fluctuates randomly around the average value Nmoy, the instantaneous rest mass $M i$ of the particle fluctuates also randomly around the average value $M$ determined previously.

As well, we cannot predict, at a precise instant, the exact value of $M i$ as it varies randomly.

But we can know the standard deviation $\sigma_{\mathrm{m}}$ of the Laplace-Gauss distribution of the elementary particles rest mass variations:

$$
\sigma_{\mathrm{m}}=\sigma_{\mathrm{n}} E u / c^{2}
$$

\section{G.5 - Matter Particles Momentum Fluctuations}

Relation (G1) gave us the linear momentum transferred to a particle of matter by a single Universon, when it is captured. Consequently, the Nmoy Universons captured, on average, simultaneously, during time $\tau$ transfer a larger linear momentum to the particle. However, these Universons are coming from all directions of space, and the natural random fluctuations are distributed as well in intensity as in direction of arrival of the free Universons. 
If the flux was constant, and distributed isotropically, in permanence, there would not be any linear momentum transferred to the matter particle at rest, on average. But the fluctuations in intensity, and in direction, of the flux transfer to the particle a momentum that fluctuates both in intensity and in direction.

These fluctuations in intensity and direction of the particle momentum are a random agitation around an average position, in all three directions of space. This is like the Brownian agitation of the dust particles in a quiet atmosphere room.

Relation (G1) corresponds to the linear momentum transferred to the matter particle by a single Universon, in any direction that is the direction of the Universon movement before its interaction. This momentum is integrally transferred to the matter particle, the momentum of which can, at each instant, be represented by three components $p x, p y$ and $p z$, according to the three axes or the observer frame of reference.

The tri-dimensional version of the Pythagoras relation allows us to write, for any captured Universon, following relation $(\mathrm{G} 1)$ :

$$
p x^{2}+p y^{2}+p z^{2}=(E u / c)^{2}
$$

But the distribution of the random fluctuations of the Universons flux is such that the flux remains, on average, strictly isotropic. This means that, on average, it is distributed equitably between the three directions of space. Consequently, on average, each component of the linear momentum should be one third of the value of the second term of expression (G11):

On average, for each captured Universon:

$$
p x^{2}=1 / 3(E u / c)^{2}
$$

So we get, for each captured Universon:

$$
p x=3^{-1 / 2} \mathrm{Eu} / \mathrm{c}
$$

In a same way, from (G8), which is the value of the standard deviation $\sigma_{\mathrm{n}}$ of the fluctuations of the number of Universons captured during time $\tau$, we can define a standard deviation $\sigma_{\mathrm{nx}}$ of the number of Universons which should have an action on the linear momentum of the matter particle along the $\mathrm{x}$ axis, and as well for the three axes:

$$
\sigma_{\mathrm{nx}}^{2}+\sigma_{\mathrm{ny}}^{2}+\sigma_{\mathrm{nz}}^{2}=\sigma_{\mathrm{n}}^{2}
$$

On average, we should get:

$$
\sigma_{\mathrm{nx}}^{2}=1 / 3 \sigma_{\mathrm{n}}^{2}
$$

So, with (G8):

$$
\sigma_{\mathrm{nx}}=3^{-1 / 2} \sigma_{\mathrm{n}}=3^{-1 / 2}\left(M c^{2} \cdot 2^{-1 / 2} / E u\right)^{1 / 2}
$$

Consequently, the standard deviation $\sigma(\mathrm{px})$ of the momentum fluctuations of the matter particle, caused by the fluctuations of the Universons flux, will be given by the product of (G13) by (G16):

$$
\sigma(\mathrm{px})=\left\{3^{-1 / 2} \mathrm{Eu} / c\right\}\left\{3^{-1 / 2}\left(M c^{2} \cdot 2^{-1 / 2} / E u\right)^{1 / 2}\right\}
$$

So:

$$
\sigma(\mathrm{px})=1 / 3\left(M E u \cdot 2^{-1 / 2}\right)^{1 / 2}
$$

By definition, this should correspond to a «variance»:

$$
\operatorname{Var}(p x)=(\sigma(p x))^{2}=1 / 9\left(M E u .2^{-1 / 2}\right)
$$

We will use relation (G18) a little further.

\section{G.6 - Matter Particles Position Fluctuations}

We have shown that fluctuations in intensity and direction of the rest particle momentum are a random agitation around an average position, in all three directions of space. This is like the Brownian agitation of the dust particles in a quiet atmosphere room.

Relation (G4) corresponds to the displacement of the matter particle caused by a single Universon, in any direction, that is the direction of the Universon movement before its capture. This movement can, at each instant, be represented by three components $d x$, $d y$ and $d z$, according to the three axes of the observer frame of reference. 
The tri-dimensional version of the Pythagoras relation allows us to write, for any captured Universon, following relation (G4):

$$
d x^{2}+d y^{2}+d z^{2}=(E u \tau / M c)^{2}
$$

But again, the distribution of the random fluctuations of the Universons flux is such that the flux remains, on average, strictly isotropic. This means that, on average, it is distributed equitably between the three directions of space. Consequently, on average, each component of the movement should be one third of the value of the second term of expression (G20):

On average, for each captured Universon:

$$
d x^{2}=1 / 3(E u \tau / M c)^{2}
$$

So we get on average, for each captured Universon:

$$
d x=3^{-1 / 2} E u \tau / M c
$$

Consequently, the standard deviation $\sigma(\mathrm{x})$ of the displacement fluctuations of the matter particle, caused by the fluctuations of the Universons flux, will be given by the product of (G22) by (G16):

$$
\sigma(\mathrm{x})=\left\{3^{-1 / 2} E u \tau / M c\right\}\left\{3^{-1 / 2}\left(M c^{2} \cdot 2^{-1 / 2} / E u\right)^{1 / 2}\right\}
$$

So:

$$
\sigma(\mathrm{x})=1 / 3\left(E u \tau^{2} \cdot 2^{-1 / 2} / M\right)^{1 / 2}
$$

By definition, this should correspond to a «variance»:

$$
\operatorname{Var}(d x)=(\sigma(\mathrm{x}))^{2}=1 / 9\left(E u \tau^{2} \cdot 2^{-1 / 2} / M\right)
$$

We will use relation (G24) down here.

\section{G.7 - Heisenberg's Relation}

German physicist Werner Heisenberg established, in 1927, the fundamental lower limit of the product of the standard deviations $\sigma(\mathrm{px}) . \sigma(\mathrm{x})$ of the momentum $\sigma(\mathrm{px})$ and of the position $\sigma(\mathrm{x})$ of an elementary matter particle (Heisenberg, 1933).

The Universons model allows us to give a physical meaning to this analysis: the quantum fluctuations of the Universons natural flux. These fluctuations agitate matter particles even if no external force is applied to them.

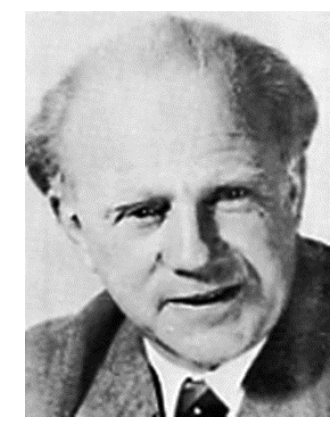

Figure G-2. Werner Heisenberg 1901 - 1976 German physicist, he was one of the founders of quantum physics. Nobel prize winner in 1932

Heisenberg's relation is quite simple:

$$
\sigma(\mathrm{px}) \cdot \sigma(\mathrm{x}) \geq h / 4 \pi
$$

In expression (G26) parameter $h$ is the «Planck's constant» which was introduced by Max Planck in 1900, in the frame of his study of the black body radiation versus its temperature. The Planck's constant allows to determine the energy of photons (Electromagnetic Energy quanta) versus their wavelength (colour). We know that:

$$
h=6,626 \cdot 10^{-34} \text { Joule. second }
$$

Let us use the values of the standard deviations previously determined by (G18) and (G24), we can write: 


$$
\sigma(\mathrm{px}) . \sigma(\mathrm{x})=1 / 3\left(M E u \cdot 2^{-1 / 2}\right)^{1 / 2} \cdot 1 / 3\left(E u \tau^{2} 2^{-1 / 2} / M\right)^{1 / 2} \geq h / 4 \pi
$$

Simplifying:

$$
1 / 9\left(E u^{2} \tau^{2} / 2\right)^{1 / 2} \geq h / 4 \pi
$$

Considering that Heisenberg minimum is given by the natural fluctuations for a particle at rest, we get again a fundamental relation we already got in Appendix A. But here it is coming from fluctuations distribution:

$$
\text { Eu } \tau \geq 2^{1 / 2} .9 h / 4 \pi \approx h
$$

Effectively, this is really a fundamental relation, also established in Appendix A, as it ties the parameters of the Universons model with the fundamental Planck's constant of quantum physics. We observe also that this relation is dimensionally correct, as we have «Joule . second» units in both members of the relation. This fundamental result allows us to determine:

$$
E u \tau \approx 6.626 \cdot 10^{-34} \text { Joule . second }
$$

We already know from Appendix B that $\mathrm{Eu}=8.5 .10^{-21}$ joule, therefore:

$$
\tau \approx 7.8 \cdot 10^{-14} \text { second }
$$

This value confirms the minimum value defined by Universons model. And this is one important theory parameter.

\section{G.8 - Fluctuations of Astronomical Gravitational Flux of Universons}

We call Astronomical Gravitational flux of Universons, the flux coming to a matter particle that has previously crossed an Astronomical mass of matter, a star mass for example.

The Natural isotropic flux of Universons average intensity is about $F_{u}=6.3 .10^{80}$ Universons per square meter, per second, distributed into the $4 \pi$ steradians directions.

This flux is fluctuating permanently around this average intensity with a standard deviation:

$$
\sigma_{v}=\mathrm{F}_{\mathrm{u}}{ }^{1 / 2}=2.5 \cdot 10^{40} \mathrm{U} \cdot \mathrm{m}^{-2} \cdot \mathrm{s}^{-1 / \text { in } 4 \pi \text { steradians directions }}
$$

We can compare this with the "white noise" fluctuations of a current of electrons of average intensity I showing a fluctuations intensity:

$$
I_{e f f}=(2 q I \Delta f)^{1 / 2}
$$

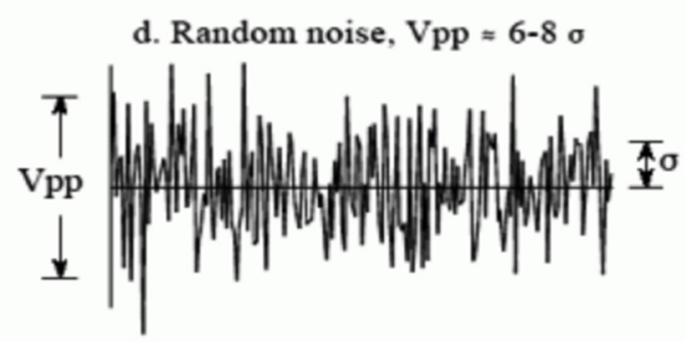

Figure G3. Example of "white noise" added to a DC current of electrons. This is a typical Quantum fluctuation.

Into that relation concerning fluctuations of a flux of electrons, I is the current intensity similar to $F_{u}$ and $q$ is the charge of one electron. This relation takes care of the bandwidth $\Delta f$ of the measuring instrument.

The same kind of situation exists for a matter particle which is concerned by the intensity of the incident Universons flux $F_{i}$ and the " bandwidth $1 / \tau$ " of the gravitational interaction with Universons.

So we can apply relation (G34) to the situation of matter particles concerned by the "efficient" intensity of a Universons flux, replacing $q$ by the energy $E u$ carried by a single Universon:

$$
F_{i}=\left(2 E u F_{u} l / \tau\right)^{1 / 2}=1.17 \cdot 10^{37} \mathrm{U} \cdot \mathrm{m}^{-2} \text { in } 4 \pi \text { steradians directions }
$$

This "efficient" value of the fluctuation intensity takes care of the limited "bandwidth" of the matter particle capture effect. 
Now let us calculate that Gravitational efficient flux intensity at different distances from a star, the Sun for example, at different distances $D$ of that star.

The value of the solid angle from which are coming the Gravitational Universons of flux $F_{i}$ is equal to the Sun surface divided by $4 \pi$ times the square of the distance $\mathrm{D}^{2}$.

$$
F_{i D}=F_{i}\left(4 \pi D^{2}\right)^{-1}=1.4169 \cdot 10^{54} \cdot D^{-2}
$$

We are going to consider classical Astronomical distances D from the Sun:

— One UA $=1.5 .10^{11} \mathrm{~m}(\mathrm{UA}=$ Astronomical Unit $)$

— One LY $=9.46 .10^{15} \mathrm{~m}$ (LY = light year)

$-5 \mathrm{LY}=4.73 .10^{16} \mathrm{~m}$

$-30000 \mathrm{LY}=2.84 .10^{20} \mathrm{~m}$ (Distance of Solar system in Galaxy)

$-50000 \mathrm{LY}=4.73 .10^{20} \mathrm{~m}$ (Radius of our Milky Way Galaxy)

And we will also calculate the flux received by an atom of $0.4 \mathrm{~nm}$ diameter during capture time $\tau$.

Table G-1. Efficient flux from fluctuations at different Astronomical distances

\begin{tabular}{lll}
\hline Distance from Sun & Efficient flux $\mathbf{F}_{\mathbf{i}}$ & Flux for one atom of $\mathbf{0 . 4} \mathbf{~ n m}(\mathbf{U})$ \\
\hline $1 \mathrm{UA}$ & $4.9 \cdot 10^{18} \mathrm{U} / \mathrm{m}^{2}$ & 0.616 \\
$1 \mathrm{LY}$ & $1.23 \cdot 10^{9} \mathrm{U} / \mathrm{m}^{2}$ & $1.55 .10^{-10}$ \\
$5 \mathrm{LY}$ & $4.94 \cdot 10^{7} \mathrm{U} / \mathrm{m}^{2}$ & $6.2 .10^{-12}$ \\
$30000 \mathrm{LY}$ & $1.37 \mathrm{U} / \mathrm{m}^{2}$ & $1.72 .10^{-19}$ \\
$50000 \mathrm{LY}$ & $0.494 \mathrm{U} / \mathrm{m}^{2}$ & $6.20 .10^{-20}$ \\
\hline
\end{tabular}

These results mean that even very close to a star of the Sun size, there is less than one Universon coming through the Sun to a distant atom.

Even if we consider a mass of 500 billions Suns (a galaxy), there is always less than a single Universon coming to a matter particle from a fluctuation.

Therefore there is a dissymmetry into the incident Universons fluxes received by a matter particle at a distant D of an Astronomical mass of matter.

- From direction of center of that mass, matter particle receives NO Universons from fluctuations.

- From opposed direction (Space) matter particle receives flux $F_{i}$ which decreases as the square of distance $D$.

This is the phenomenon explaining Gravitation.

\section{Gravitation is caused by fluctuations of the natural isotropic flux of Universons.}

This is a really fundamental prediction from our model.

\section{Appendix H - Quantum Model of Gravitation}

\section{H.1 - Cause of Gravitation Acceleration}

We have shown in Appendix $G$ that the Natural Isotropic flux of Universons is permanently fluctuating in intensity and in direction. These fluctuations are random and follow the Laplace-Gauss statistical distribution.

Let us reproduce here the table calculated into Appendix G:

Table H-1. Intensity of efficient flux from a random fluctuation, at a distance D from a Sun like star

\begin{tabular}{lll}
\hline Distance from Sun & Efficient flux $\mathbf{F}_{\mathbf{i}}$ & Flux for one atom of $\mathbf{0 . 4} \mathbf{~ n m}(\mathbf{U})$ \\
\hline $1 \mathrm{UA}$ & $4.9 \cdot 10^{18} \mathrm{U} / \mathrm{m}^{2}$ & 0.616 \\
$1 \mathrm{LY}$ & $1.23 \cdot 10^{9} \mathrm{U} / \mathrm{m}^{2}$ & $1.55 .10^{-10}$ \\
$5 \mathrm{LY}$ & $4.94 \cdot 10^{7} \mathrm{U} / \mathrm{m}^{2}$ & $6.2 .10^{-12}$ \\
$30000 \mathrm{LY}$ & $1.37 \mathrm{U} / \mathrm{m}^{2}$ & $1.72 .10^{-19}$ \\
$50000 \mathrm{LY}$ & $0.494 \mathrm{U} / \mathrm{m}^{2}$ & $6.20 .10^{-20}$ \\
\hline
\end{tabular}


These results mean that even very close to a star of the Sun mass and size, there is less than one Universon coming through the Sun to a distant atom of $0.4 \mathrm{~nm}$ diameter.

The "efficient Gravitational flux" is the one interacting with a matter particle during capture time $\tau$ and that has first crossed the distant star matter.

The Gravitational efficient flux coming from fluctuations, in all directions of space, in the direction opposed to the star has an intensity (Appendix G):

$$
F_{i}=\left(2 E u F_{u} 1 / \tau\right)^{1 / 2}=1 \cdot 17 \cdot 10^{37} \mathrm{U} \cdot \mathrm{m}^{-2} \text { in } 4 \pi \text { steradians directions }
$$

This "efficient" value of the fluctuation intensity takes care of the limited "bandwidth" of the matter particle capture effect.

These fluctuations must be very fast as they are part of a general flux of $6.3 .10^{80}$ Universons per second, per square meter into the $4 \pi$ steradians directions. Effectively a fluctuation of $10^{37}$ Universons should not exist anymore longer than $10^{37} / 6.3 .10^{80}=1.58 \cdot 10^{-43}$ second.

This is much shorter than the interaction time duration of $7.8 .10^{-14}$ second for a single Universon.

This means that each Universon coming from a so brief fluctuation is mixed with about $5.10^{29}$ other Universons which are coming from the isotropic flux component. So the momentum direction of the single fluctuation Universon is really "lost" into the interaction effect on trajectories.

So matter is a kind of "filter" for these fluctuations, they are not transmitted.

It is not true for fluctuations that have not crossed a mass of matter.

Therefore, on the Earth surface, a matter particle receives fluctuations from the Zenith direction, but none from the Nadir direction.

So fluctuations are no more symmetric, there is a larger average flux from the Zenith than from the Nadir direction. The difference is small, which explains why the gravitational constant $\mathrm{G}$ has a so low value.

We can take care of that effect by including it into a "virtual capture cross section $S$ " for Universons coming from the distant mass where the flux intensity is reduced. This is an "apparent or virtual" absorption, not a real one, as Universons are not absorbed by matter particles.

This way we are able to calculate the Gravitation force from our quantum Inertia model.

\section{H.2 - Gravitational Force and Gravitation Model}

We calculate the gravitational force between two masses $\mathrm{M}_{1}$ and $\mathrm{M}_{2}$.

The intensity of the natural flux of Universons being $F_{u}$ and $S$ being the virtual interaction cross section, using the model notations, the number of Universons $n$ captured by the first body of mass $M_{l}$, each second, from the $4 \pi$ steradians, is equal to:

$$
n=F_{u} S M_{1}
$$

All these Universons are re-emitted. At a distance $D$ from the mass $M_{l}$, these re-emitted Universons propagate and are distributed along the surface of a sphere of radius $D$; so, at the surface of the sphere, the flux $F^{\prime}$ of these Universons is given by:

$$
F^{\prime}=n / 4 \pi D^{2}=F_{u} S M_{1} / 4 \pi D^{2}
$$

So $F^{\prime}$ is the flux of Universons, first captured by $M_{l}$ and arriving in the solid angle $\Omega$ on mass $M_{2}$. These Universons are partially captured by the particles of the second body of mass $M_{2}$ and the Universons number $n$ ' that are captured, during capture time $\tau$, is:

$$
\begin{gathered}
n^{\prime}=F^{\prime} S M_{2} \tau \\
n^{\prime}=F_{u} S^{2} M_{I} M_{2} \tau / 4 \pi D^{2}
\end{gathered}
$$

These n', two times captured Universons, transfer to matter, when re-emitted, a momentum which is not compensated in the other direction.

So, they create a resultant force (gravitation force) which is oriented towards the incoming flux, or the direction of the mass $M_{l}$. The force exerted by each Universon being $E_{1} / c \tau$ the value of this resultant force is:

$$
\text { Force }=n^{\prime} E_{1} / c \tau
$$




$$
\text { Force }=F_{u} S^{2} M_{1} M_{2} E_{1} / 4 \pi c D^{2}
$$

This can be written:

$$
\text { Force }=G M M^{\prime} / D^{2}
$$

With:

$$
G=F_{u} S^{2} E_{1} / 4 \pi c
$$

Expression (H7) is the well known Newton's law of Gravitation, and expression (H8) ties the gravitational constant $G$ to the parameters of our model, mainly the parameters of the natural flux of Universons.

From our model we know that:

$$
\tau S F_{u} E_{1} / c^{2}=1
$$

So expression (H8) can be written also:

$$
\boldsymbol{G}=\boldsymbol{S} \boldsymbol{c} / 4 \boldsymbol{\pi} \tau
$$

Expression (H10) ties the gravitational constant $G$ to the parameters of the Universons captured by matter. Both expressions (H8) and (H10) are equivalent.

We should note that $S$ is a "virtual" cross section into that model.

\section{Appendix $\mathbf{J}$ - Gravitation Acceleration at Very Large Distances between Masses}

\section{J.1 - Introduction}

Our model of Gravitation says that it is caused by Quantum fluctuations of the Natural isotropic flux of Universons. There is a very brief duration of such fluctuations, so the captured Universons of the increased flux, during a fluctuation, are dispersed at re-emission. Therefore a distant matter particle is submitted to a flux intensity difference between the one coming from free space directions, and the one coming through a distant mass of matter. These facts are demonstrated into Appendixes $\mathrm{G}$ and $\mathrm{H}$.

Moreover, the Universe expansion has a particular importance in the Universons theory, because any acceleration $A$ of matter is accompanied by a supplementary acceleration $H_{0} c$, oriented in the same direction as the acceleration $A$ applied on matter by an external cause, Gravitation included.

The phenomenon manifests itself for any Newtonian gravitational acceleration $A_{N}$ such as:

$$
A_{N}=G M_{G} / D^{2}
$$

Where $M_{G}$ is the «source» mass of the gravitational acceleration, situated at a distance $D$, and where $G$ is the universal gravitational constant.

So, in fact, in the Universons model, the real gravitational acceleration $A_{G}$ is not equal to $A_{N}$ but to:

$$
A_{G}=H_{0} c+A_{N}=H_{0} c+G M_{G} / D^{2}
$$

Moreover, in this model, the Newtonian gravitational acceleration $A_{N}$ is caused by a flux of Universons object of quantum fluctuations. The universal gravitational «constant» $G$ is proportional to the natural Universons flux intensity, so it is also sensitive to the quantum fluctuations of the flux.

Consequently, the Newtonian gravitational acceleration $A_{N}$, which is proportional to $G$ is submitted to the same quantum fluctuations.

These quantum fluctuations follow the Laplace-Gauss statistics, this means that they are defined by two parameters: on the one hand, by the mean value $A_{N}$ and, on the other hand, by the standard deviation $\sigma_{N}$ which is equal, in this case, to:

$$
\sigma_{N}=\left(A_{N}\right)^{1 / 2}
$$

By the same way, the acceleration $H_{0} c$ is submitted to the local quantum fluctuations of the Universons captures from the flux, which is the cause of the Newtonian gravitational acceleration. These fluctuations are also following the Laplace-Gauss statistics, which means that they are also defined by a mean value $H_{0} c$ and a standard deviation $\sigma_{H}$ which is equal to:

$$
\sigma_{H}=\left(H_{0} c\right)^{1 / 2}
$$


Nevertheless, quantum fluctuations of $A_{N}$ and of $H_{0} c$ are caused by local phenomena separated by the distance $D$. Consequently, these quantum fluctuations cannot be synchronous. One must say that the two fluctuations are independent. This point is important to consider.

\section{J.2 - Standard Deviation of Real Acceleration AG}

When Newtonian gravitational acceleration is much larger than acceleration $H_{0} c$, the real gravitational acceleration $A_{G}$ is also submitted to quantum fluctuations following the Laplace-Gauss statistics. Its histogram is has a bell shaped curve named "Standard distribution". This is illustrated by curve A on figure J-1. These statistics have a probability density function which is:

$$
f(A)=(0,399 / \sigma) \exp \left\{(\tilde{A}-A) /\left(2 \sigma^{2}\right)\right\}
$$

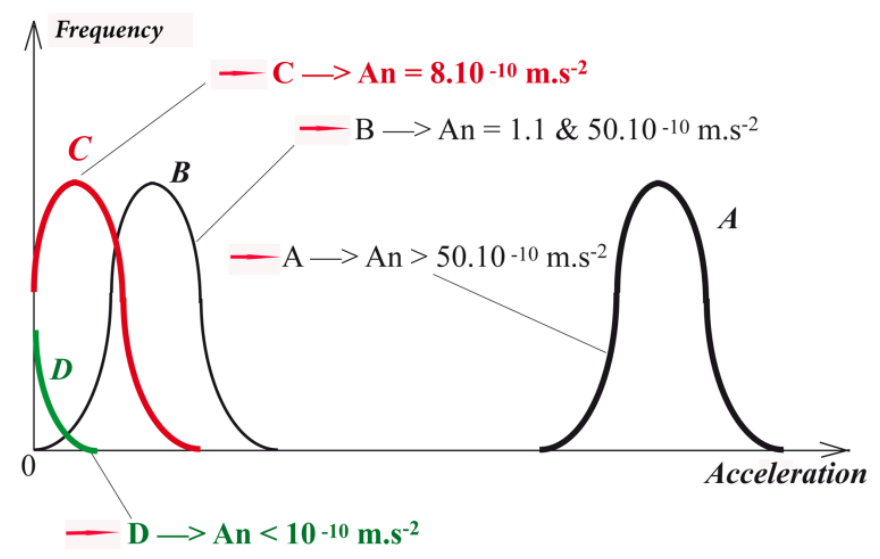

Figure J-1. Histograms of the real gravitational acceleration for several values of the Newtonian gravitational acceleration An, compared to the value of $\mathrm{H}_{0} \mathrm{c}$

Where the mean acceleration is $\tilde{A}$ and the standard deviation is $\sigma$.

The mean acceleration value is given by:

$$
\tilde{A}=A_{N}+H_{0} c
$$

And the standard deviation $\sigma$ is the one of the sum of two accelerations with independent quantum fluctuations. For two independent fluctuations, the standard deviation is equal to the product of the two standard deviations of the accelerations:

$$
\sigma_{G}=\sigma_{N} \sigma_{H}=\left(A_{N} H_{0} c\right)^{1 / 2}
$$

\section{J3 - Laplace-Gauss' or Poisson's Statistics?}

Let us consider what happens when the Newtonian gravitational acceleration amplitude is reduced. The mean value of the real gravitational acceleration, which is the position of the maximum of its histogram, is evidently reduced also.

It moves towards the origin, from A to B in figure J-1. The practical half width of the gauss curve of the histogram is about three times the standard deviation $\sigma$. So, a continuous reduction of the mean Newtonian acceleration An, caused by the increase of distance $D$ between the two masses, can bring the Gauss curve to position $\mathrm{B}$, where the left wing foot of the curve is exactly at zero acceleration for 3 standard deviations under the mean acceleration.

This means simply that the real gravitational acceleration will be nil for about $1 \%$ of the time when:

$$
\tilde{A}=3 \sigma_{G}=3\left(A_{N} H_{0} c\right)^{1 / 2}
$$

And with (J6):

$$
A_{N}+H c=3\left(A_{N} H_{0} c\right)^{1 / 2}
$$

This relation has two roots:

$$
A_{N 1}=0,15 H_{0} c \text { AND } A_{N 2}=6,85 H_{0} c
$$


With $H_{0} c=7.1 .10^{-10} \mathrm{~m} / \mathrm{s}^{2}$ (Pioneer constant acceleration):

$$
A_{N l}=1.1 \cdot 10^{-10} \mathrm{~m} / \mathrm{s}^{2} \text { AND } A_{N 2}=5 \cdot 10^{-9} \mathrm{~m} / \mathrm{s}^{2}
$$

So, when the Newtonian gravitational acceleration reaches these very small amplitudes, the histogram of the real gravitational acceleration reaches precisely position B on Figure J-1.

On the other hand, when the Newtonian gravitational acceleration is larger than $A_{N 2}$ then the histogram is situated far from the origin, like in position A of Figure J-1.

What happens when Newtonian acceleration is between the two preceding values, for example $A_{N}=H_{0} c$ ? Then, the histogram of the real gravitational acceleration, composed of $A_{N}$ and $H_{0} c$ moves towards the origin, as in the case $\mathrm{C}$ of Figure $\mathrm{J}-1$, and the real acceleration becomes nil when the instantaneous acceleration is smaller than one standard deviation under the summit value of the bell shaped curve.

However, there are never negative values of gravitational accelerations. Effectively, the Newtonian gravitational acceleration is caused by a supplementary flux of Universons coming from the direction of the center of mass of the second body. If the acceleration had been allowed to become negative, this would mean that the corresponding flux would have to come from an opposed direction, from a direction where there is no other mass, which is evidently impossible. For this reason, the resultant acceleration can become nil but is not allowed to become negative. This is what is illustrated on Figure J-1.

So, when $A_{N}<10^{-10} \mathrm{~m} / \mathrm{s}^{2}$ the real gravitational acceleration has an histogram analog to case $\mathrm{D}$ in Figure J-1. Evidently, histograms of types $\mathrm{C}$ or D do not correspond to a distribution of the quantum fluctuations defined by the Laplace-Gauss statistics. These histograms are of the Poisson's type statistics. The Poisson's statistics probability density being represented by:

$$
f(n)=\left(\lambda^{n} / n !\right) e^{-\lambda}
$$

Where $n$ is the number of Universons involved in the inception of the Newtonian gravitational acceleration, number which is proportional to $A_{N}$ and where the $\lambda$ parameter replaces the standard deviation, with:

$$
\lambda=\left(A_{N} H_{0} c\right)^{1 / 2}
$$

However the mean value of the acceleration, in a Poisson's distribution of the quantum fluctuations, is absolutely not the same value as in a Laplace-Gauss' distribution. This appears evident when looking at curves $\mathrm{A}$ and $\mathrm{D}$ in Figure J-1. In the Poisson's distribution, the mean value of the acceleration is the value of the parameter $\lambda$ of the distribution.

So, when the Newtonian gravitational acceleration becomes smaller than the preceding boundaries, the mean value of the real gravitational acceleration becomes equal to:

$$
A_{G}=\lambda=\left(A_{N} H_{0} c\right)^{1 / 2}
$$

\section{J.3 - Summary of Consquences (Figure J-2)}

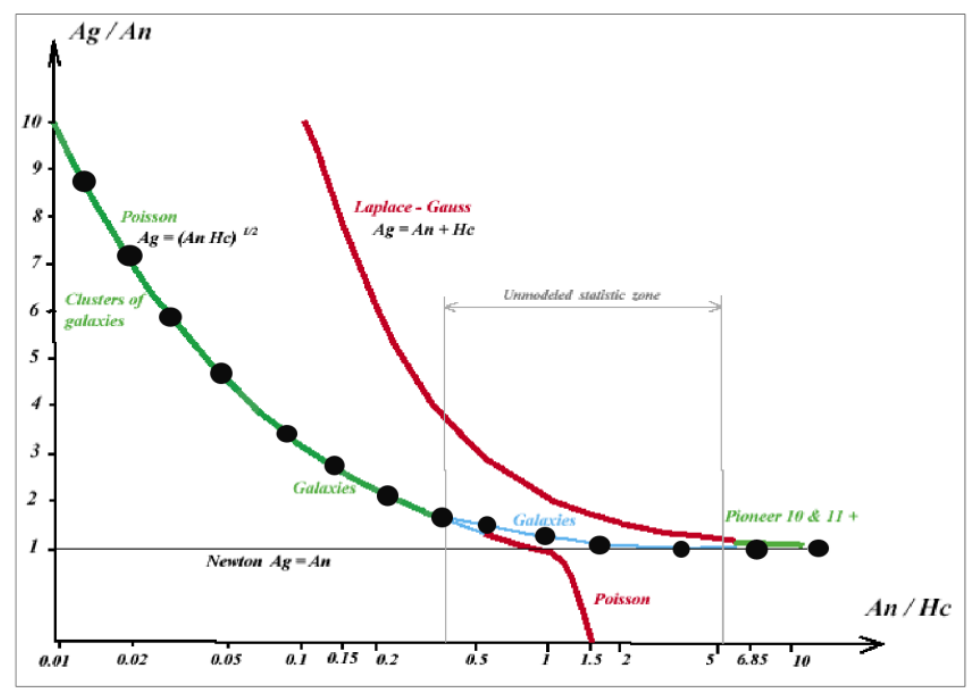

Figure J-2. Evolution of predicted ratio Ag / An versus ratio $\mathrm{An} / \mathrm{H}_{0} \mathrm{c}$ for large distances. All galaxies Gravitational acceleration values are situated onto black dots curves 
When the Newtonian gravitational acceleration $A_{N}$ is larger than $5.10^{-9} \mathrm{~m} / \mathrm{s}^{2}$ then the mean real gravitational acceleration $A_{G}$ is equal to:

$$
A_{G}=A_{N}+H_{0} \mathrm{C}
$$

When the Newtonian gravitational acceleration $A_{N}$ is smaller than $10^{-10} \mathrm{~m} / \mathrm{s}^{2}$ then the mean real gravitational acceleration $A_{G}$ is equal to:

$$
A_{G}=\left(A_{N} H_{0} c\right)^{1 / 2}
$$

When the Newtonian gravitational acceleration amplitude $A_{N}$ is comprised between $10^{-10} \mathrm{~m} / \mathrm{s}^{2}$ and $5.10^{-9} \mathrm{~m} / \mathrm{s}^{2}$ then the mean real gravitational acceleration $A_{G}$ is not modelled by the Laplace-Gauss' statistics, and neither modelled by the Poisson's statistics. Nevertheless, it appears, experimentally approximately modelled, in this small zone, by the following hybrid equation:

$$
A_{G}=A_{N}+\left(A_{N} H_{0} c\right)^{1 / 2}
$$

This is a combination of expressions (J15) and (J16), in the relatively fuzzy zone where the two types of statistics are at the limit of their mathematical validity. These considerations are summarized in figure J-2, where the experimental confirmations are also indicated as black dots.

Laplace-Gauss' statistics is the limit of the Poisson's statistics, when the number of Universons involved in the Newtonian gravitational acceleration becomes very large. So it is normal to have to change the type of distribution when the number of Universons involved is largely modified. The Laplace-Gauss' statistics concerns continuous phenomena or large samples, while the Poisson's statistics concerns quantified phenomena with few samples.

\section{J.4 - Our Results Predict the Dynamic Behaviour of Galaxies}

We are going to show now, by an example, that the validity limits of Gauss' and Poisson's statistics in the accelerations quantum fluctuations have to be applied when considering the dynamic behaviour of astronomical objects, such as spiral galaxies where external stars are very distant from the galactic center where mass is concentrated (Rubin \& al., 1980).

Let us consider a spiral galaxy with a central bulb mass of $5.10^{40} \mathrm{~kg}$. This corresponds to a matter mass of about 25 billions solar type stars, a very classical stellar density in central region of such galaxies.

One can calculate, from expression (J1), that the Newtonian gravitational acceleration reaches the preceding validity limits when the distance $D$ of stars, from the galactic center, is equal to:

$$
D_{1}=5800 \text { parsecs }
$$

Then for

$$
D_{2}=860 \text { parsecs }
$$

These are distances very close to the galactic center, because most of these galaxies have a total diameter of the order of 30000 parsecs. (One parsec distance is equal to $3.10^{16}$ meters).

Consequently, from our predictions, the quasi totality of the stars in galactic discs of spiral galaxies are submitted to a real gravitational acceleration defined by expression (J16) and not by the Newton's relation (J1).

The dynamic behaviour of these galaxies must be largely affected by this result. Let us check that.

\section{J.5 - Universons Model and Tully-Fisher's Law}

Our Universons model of Gravitation predicts that the quasi totality of the stars of the galactic discs of spiral galaxies are submitted to a real gravitational acceleration defined by expression (J16). Bringing in this relation the value of $A_{N}$ defined by expression (J1), we get:

$$
A_{G}=\left(G M_{G} H_{0} c\right)^{1 / 2} / D
$$

The circular orbital speed of the stars $V$ is such that the centrifuge acceleration compensates exactly the real gravitational acceleration, so:

$$
V^{2} / D=A_{G}=\left(G M_{G} H_{0} c\right)^{1 / 2} / D
$$

And it comes:

$$
V^{2}=\left(G M_{G} H_{0} c\right)^{1 / 2}
$$


So:

$$
V^{4}=G M_{G} H_{0} c
$$

This expression is simply the Tully-Fisher's relation for spiral galaxies (Tully \& Fisher, 1977), a very well known law used by Astronomers (Figure J-3), discovered experimentally, from the observation of many rotation curves of galaxies and later justified by the "Viriel" theorem. But, here this empirical relation is fully justified.

\section{Therefore our model prediction is confirmed.}

In fact, the original Tully-Fisher's relation was between the luminosity of the galaxies and their rotation speed. But there is a mean relation between the luminosity and the mass of the stars in a galaxy. The Tully-Fisher's relation attributed a different proportionality constant between $V^{4}$ and the luminosity. This means that the Tully-Fisher proportionality constant reflected the evolution age of the stars in each type of galaxy. But in the real Tully-Fisher's relation (J23), it is mainly the stars mass that is acting, because these galaxies are relatively poor in gas mass, generally less than $20 \%$.

Expression (J23) demonstrates also that the orbital speed of stars, in the galactic disc, is independent of the galactic radius. This is confirmed by all observations, since more than 50 years, but this was unexplained until now, and gave the hypothesis of Dark Matter presence.

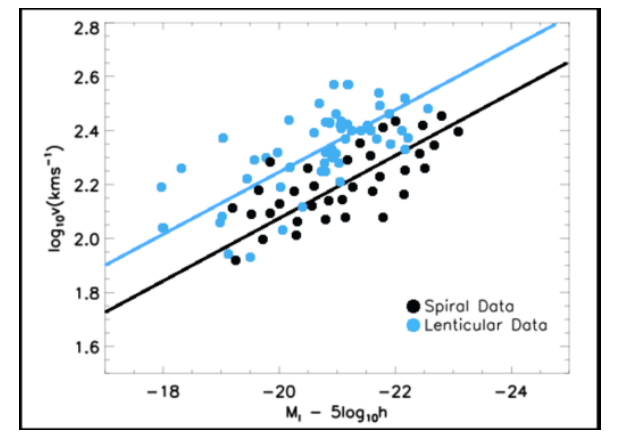

Figure J-3. Empirical Tully-Fisher's law for spiral and lenticular galaxies.

\section{J.6 - Not Any "Dark Matter" Mass Is Required}

Expression (J23) does not use any Dark Matter mass to explain the quasi-constant orbital speed of stars in the disc of spiral galaxies. This confirms the fact that the hypothetic "Dark Matter" has never been put into observational evidence.

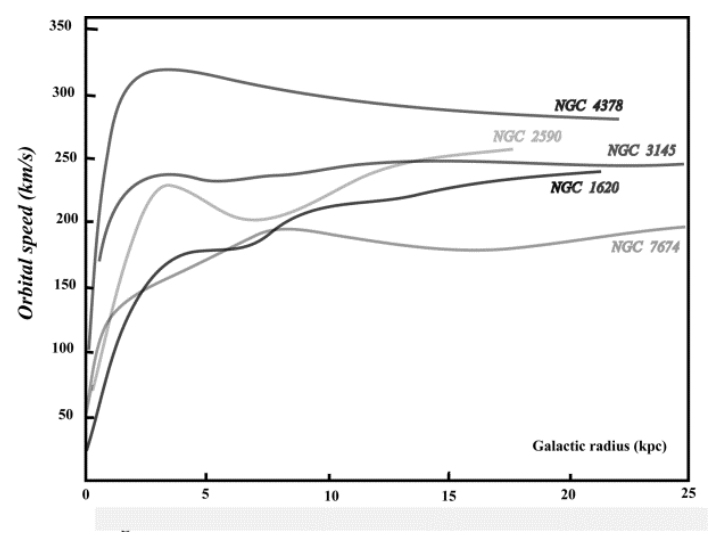

Figure J-4. Observed orbital velocities in spiral galaxies

Let us consider a simple example, with a spiral galaxy composed of a hundred billions stars of the solar type (2.10 ${ }^{30} \mathrm{~kg}$ ) and no gas. Its total luminosity would be comparable to the luminosity of the brightest spiral galaxies really observed. Expression (J23) tells us that the orbital rotation speed of the stars in the disc of such a galaxy should be $314 \mathrm{~km} / \mathrm{s}$, an order of magnitude in the range of the rotation speeds observed. 
On the other hand, if such a galaxy had a total mass ten times larger, as it is suggested by the dark matter hypothesis, its rotation speed would be, according to (J23), equal to $560 \mathrm{~km} / \mathrm{s}$, a value never observed. The observed rotation speeds of the real spiral galaxies are between 100 and $300 \mathrm{~km} / \mathrm{s}$ (Figure J-4).

Therefore our model gives a physical support to the MOND paradigm (Milgrom 2020), it explains the orbital velocity of stars in galaxies (Rubin \& al. 1980), it explains the Tully-Fisher's relation (Tully \& al. 1977) and rules out the need for Dark Matter hypothesis (CERN 2012). So Dark Energy is concerned (NASA 2015).

\section{J.7 - Computer Verification of the Effect of Quantum Fluctuations}

This verification is based on the observation of spiral galaxies. As we have shown, the Newtonian gravitational acceleration $A_{N}$ of stars, in the discs of these galaxies is largely smaller than $H_{0} c$. This acceleration $A_{N}$ is larger than $H_{0} c$ only for stars orbiting at a distance smaller than about $10 \%$ of the total galactic radius. So, relation (J22) must apply for about $90 \%$ of the galactic discs.

This means that the orbital speed of stars, in this region, must be quasi independent of their orbital radius, a fact confirmed by observations since a long time, and illustrated by figure J-4 above.

Moreover, the "plateau speed" must be roughly proportional to the fourth root of the total mass of each galaxy. This means that each individual galaxy must have its own "plateau speed" of rotation. This is also very clear in the preceding observations.

Finally, because the orbital speed is proportional to the fourth root of the total mass, one must observe a relative modest dispersion of the rotation speeds between the different galaxies. In the preceding figure J-4 the speed dispersion is of a factor 1.6, implying a mass dispersion of a factor 8 between the galaxies of this sample, a dispersion that is confirmed by the ratio of their relative luminosity. The galaxies of the sample used are the closest, so their rotation curve and luminosity is the most accurately known.

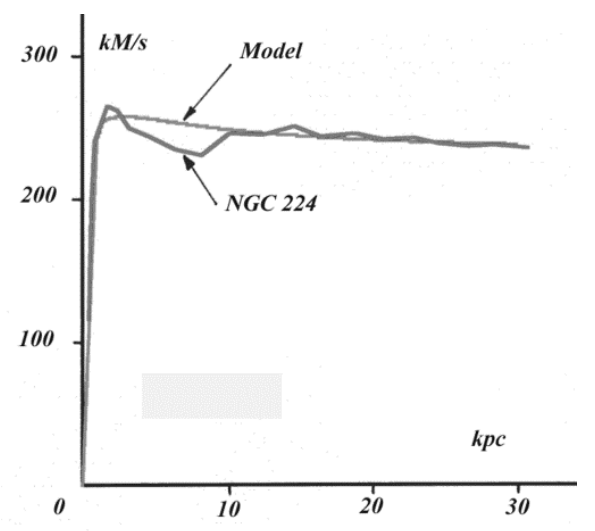

Figure J-5. Calculation result of orbital velocities into galaxy NGC 224 from our model

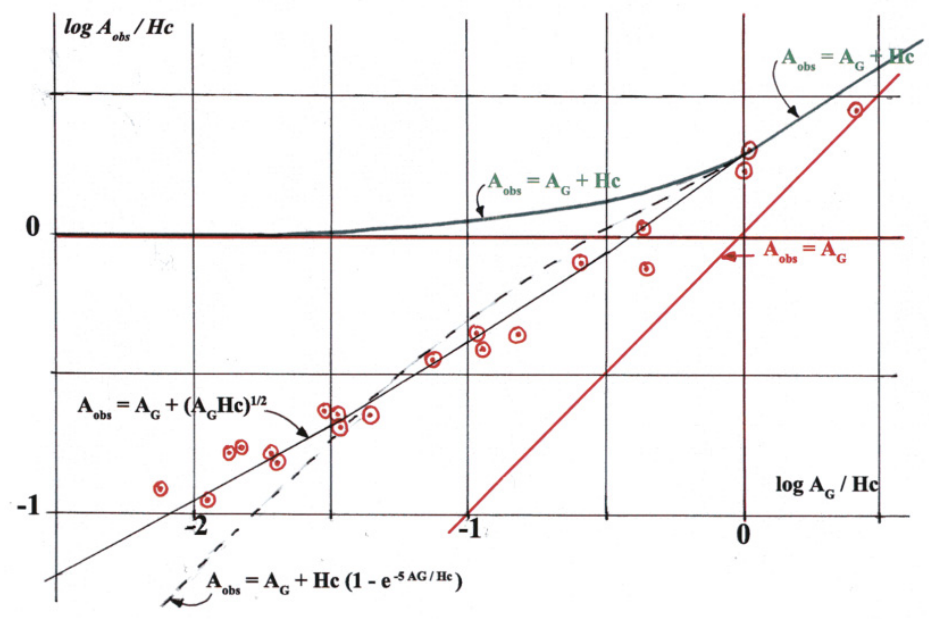

Figure J-6. Red dots are real accelerations observed into real spiral galaxies, This confirms also our model 
One can complete this verification with the help of a computer simulation, in order to calculate the orbital speed of stars all along the galactic radius, taking into account the real distribution of mass, deduced from the observed distribution of luminosity of the stars along the galactic radius.

This is what has been done for NGC 224 galaxy, in figure J-5, on the basis of expression (J22).

This very simple simulation does not take into account the local variations of the stars density in the spiral arms of the real galaxy, which explains the undulations of the real velocities curve as compared to the simulated one. But it is quite clear that the simulation result is correct.

These demonstrative results show that the quantum fluctuations of the Universons theory are able to explain the dynamic behaviour of spiral galaxies, without any call to the Dark Matter hypothesis. Effectively, the previous computer simulation uses only the stars and gas mass, in expression (J22), to calculate the rotation velocity of matter in the galaxy.

\section{J.8 - Conclusions of Appendix $\mathbf{J}$}

The Universons model, which is a Quantum Gravitation model, is the modernized, and largely improved version of ideas emitted for the first time during Newton's life epoch. They were championed by Descartes, and by Georges-Louis Lesage in 1758.

These ideas have preoccupied the most eminent members of French Sciences Academy during many years, as well as numerous European scientists. Then they have been abandoned well after Newton has published his gravitation theory in 1687.

The General Relativity theory seems to have then prohibited their study.

But now, the same General Relativity theory offers a solution, at very large spatio-temporal scale, where there appears to exist a pressure exerted by Gravitation on matter everywhere, precisely like the pressure exerted by the Universons on matter at quantum scale. This is the Robertson-Walker's model proposed by physicist Patrick Marquet, (Poher \& Marquet, 2012) with a Weyl curvature.

Precisely, neither Descartes, nor Lesage, or the members of the Sciences Academy of this epoch, had the relativistic knowledge necessary to modify the basic quantization hypotheses of Gravitation and render them compatible with observation facts.

However, this is possible nowadays and the results obtained are quite interesting to consider.

First, this model becomes compatible with the Inertia principle, that was not the case in its first hypotheses during the Newton's epoch.

But, much more important, it demonstrates the Einstein's equivalence principle, as Inertia and Gravitation are shown to be the same phenomenon.

Second, the modified theory predicts a lot of new facts.

And these facts are effectively observed. They are tied to the Universe expansion and the Quantum fluctuations associated with the gravitation quantization.

Thanks to these predictions, quite old observations results, unexplained until now, find simple and evident justifications without calling for new physics or strange and unobserved Dark Matter.

These are the quasi-constant orbital speed of stars in spiral galaxies, the proper speed of the galaxies in clusters, or the observed constant supplementary acceleration of all distant interplanetary spacecraft.

The effective confirmation of all these predictions should logically ask for a new approach of all dynamic models at very low accelerations. Even our solar system orbital models are concerned when high accuracy is needed.

Evidently, these predictions have also important cosmological consequences, because they wipe out the existence of the dark matter hypothesis, concerning the main constituent of the Universe.

The famous $\Lambda \mathrm{CDM}$ concordance model has no more reason to be considered.

However, it is quite clear than there remains a lot of work to be done in order to insert the quantum Universons model into General Relativity. The relation between the Quantum world and the Universe at large scale, particularly the proven curvature of space-time, remains an enigma. 


\section{Appendix $\mathrm{K}$ - Comparison of $\mathrm{H}_{0} \mathrm{C}$ with Accelerations in the Universe}

\section{K.1 - Comparison with Solar System accelerations}

Solar mass is $\mathrm{M}_{\mathrm{S}}=1.989 .10^{30} \mathrm{~kg}$, The gravitational Solar acceleration $\mathrm{A}_{\mathrm{S}}$ at a distance $\mathrm{D}_{\mathrm{S}}$ is equal to:

$$
A_{S}=G M_{S} / D_{S}^{2}=1.33 .10^{20} / D_{S}^{2}
$$

Acceleration As is equal to $H_{0} c$ at a distance from the Sun equal to:

$$
D_{S}=4.27 .10^{14} \mathrm{~m} \text { Or } 2855 \mathbf{A U}
$$

An Astronomical Unit $\left(\mathrm{AU}=1.49598 .10^{11} \mathrm{~m}\right)$ is the Earth - Sun distance, so the predicted acceleration increase $\mathrm{A}_{\mathrm{s}}+\mathrm{H}_{0} \mathrm{c}$ should manifest itself inside the Solar system, particularly for all interplanetary spacecraft.

\section{K.2 - Comparison with Milky Way Galaxy accelerations}

The mass of our Milky Way Galaxy is $\mathrm{M}_{\mathrm{G}}=2.78 .10^{41} \mathrm{~kg}$. The gravitational galactic acceleration $\mathrm{A}_{\mathrm{G}}$ at a distance $\mathrm{D}_{\mathrm{G}}$ from the galactic center is:

$$
A_{G}=G M_{G} / D_{G}^{2}=1.86 \cdot 10^{31} / D_{G}^{2}
$$

A Galactic gravitational acceleration equal to $H_{0} c$ is reached at a distance from the galactic center of:

$$
D_{G}=1.59 .10^{20} \mathrm{~m} \text { or about } 5.13 \mathrm{kpc}
$$

(1 kpc - kilo parsec $\left.=3.09 .10^{19} \mathrm{~m}\right)$.

This distance is much smaller than the total radius of the Galaxy, larger than $30 \mathrm{kpc}$. So, the gravitational mass we have to consider when the gravitational acceleration is equal to $\mathrm{H}_{0} \mathrm{c}$ is much smaller than the total mass of the Galaxy. Consequently, $\mathrm{A}_{\mathrm{G}}=\mathrm{H}_{0} \mathrm{c}$ is reached much closer than $5 \mathrm{kpc}$ from the Galactic center.

Therefore, the fact that galactic Gravitational acceleration is doubled when $\mathrm{A}_{\mathrm{G}}=\mathrm{H}_{0} \mathrm{c}$ and the modification of the supplementary acceleration when $A<H_{0} c$ should be observed in $\boldsymbol{A L L}$ galaxies.

\begin{tabular}{|c|c|c|c|c|}
\hline Galaxy & NGC 224 & NGC 3145 & NGC 2590 & NGC 4378 \\
\hline $\mathrm{V} \max (\mathrm{km} / \mathrm{s})$ & 261 & 237 & 231 & 320 \\
\hline $\mathrm{R} \max (\mathrm{kpc})$ & 1.38 & 1.14 & 1.08 & 2.08 \\
\hline \multicolumn{5}{|c|}{ Distance $R=1 \mathrm{Kpc}$} \\
\hline$V$ measured & - & 204 & 127 & 257 \\
\hline Aobs / $\mathrm{H}_{0} \mathrm{c}$ & - & 1.68 & 0.65 & 2.67 \\
\hline $\mathrm{A}_{\mathrm{G}} / \mathrm{H}_{0} \mathrm{c}$ & - & 1.03 & 1.1 & 2.56 \\
\hline \multicolumn{5}{|c|}{ Distance $R=2$ Kpc } \\
\hline $\mathrm{V}$ measured & - & 231 & 195 & 313 \\
\hline Aobs $/ \mathrm{H}_{0} \mathrm{c}$ & - & 1.08 & 0.77 & 1.98 \\
\hline $\mathrm{A}_{\mathrm{G}} / \mathrm{H}_{0} \mathrm{c}$ & - & 0.429 & 0.45 & 1.05 \\
\hline \multicolumn{5}{|c|}{ Distance $R=5$ Kpc } \\
\hline $\mathrm{V}$ measured & 234 & 234 & 218 & 316 \\
\hline Aobs / $\mathrm{H}_{0} \mathrm{c}$ & 0.44 & 0.44 & 0.39 & 0.81 \\
\hline$A_{G} / H_{0} c$ & 0.153 & 0.108 & 0.114 & 0.258 \\
\hline \multicolumn{5}{|c|}{ Distance $R=10 \mathrm{Kpc}$} \\
\hline $\mathrm{V}$ measured & 238 & 242 & 221 & 297 \\
\hline Aobs $/ \mathrm{H}_{0} \mathrm{c}$ & 0.23 & 0.237 & 0.198 & 0.358 \\
\hline $\mathrm{A}_{\mathrm{G}} / \mathrm{H}_{0} \mathrm{c}$ & 0.043 & 0.03 & 0.033 & 0.073 \\
\hline \multicolumn{5}{|c|}{ Distance $R=15 \mathrm{Kpc}$} \\
\hline $\mathrm{V}$ measured & 238 & 247 & 252 & 287 \\
\hline Aobs $/ \mathrm{H}_{0} \mathrm{c}$ & 0.15 & 0.165 & 0.17 & 0.22 \\
\hline & 0.0196 & 0.014 & 0.015 & 0.033 \\
\hline \multicolumn{5}{|c|}{ Distance $R=20 \mathrm{Kpc}$} \\
\hline $\mathrm{V}$ measured & 234 & 246 & - & 282 \\
\hline Aobs / $\mathrm{H}_{0} \mathrm{c}$ & 0.11 & 0.123 & - & 0.16 \\
\hline $\mathrm{A}_{\mathrm{G}} / \mathrm{H}_{0} \mathrm{c}$ & 0.011 & 0.0077 & - & 0.019 \\
\hline
\end{tabular}

K.3 - Astronomical Total Accelerations in Galaxies When A $<\mathbf{H}_{0} \mathbf{c}$ (Stars Far Away from Galactic Center)

Table K1. Of observed Gravitational accelerations (Aobs) observations results for four spiral galaxies 
In order to examine the REAL evolution of the total Gravitational acceleration when the Newtonian gravitational acceleration $\mathrm{A}_{\mathrm{G}}$ is lower than the value $\mathrm{H}_{0} \mathrm{c}$, we can use results of observations of the orbital speed of stars in spiral galaxies. We know that the Newtonian gravitational acceleration $\mathrm{A}_{\mathrm{G}}$ is lower than $\mathrm{H}_{0} \mathrm{c}$ in most of their galactic disc.

From that data we get these red dots into a diagram:

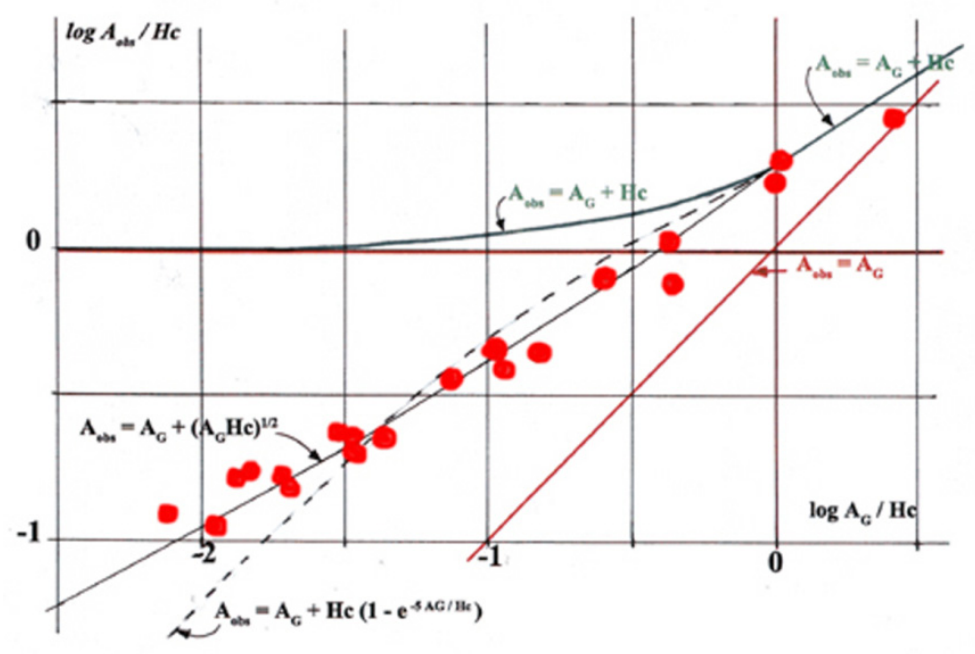

Figure K-1. Table K1 real accelerations (red dots) compared to other models (log scales)

\section{K.4 - Discussion}

We see that real values of orbital Gravitational accelerations of stars in all galaxies confirm the model:

$$
\text { Aobs }=A_{G}+\left(A_{G} H_{0} c\right)^{1 / 2}
$$

They do not follow models $\left[A o b s=A_{G}\right]$, or $\left[A o b s=A_{G}+H_{0} c\right]$ or the exponential model.

The Newtonian gravitational acceleration $\mathrm{A}_{\mathrm{G}}$ of each star is obtained from:

$$
A_{G}=G M g / R^{2}
$$

In which $G$ is the gravitational constant, and $M \mathrm{~g}$ the gravitational mass of the galaxy, corresponding to radius $R$. Mass $M g$ varies with orbital radius $R$.

One can use a sufficiently accurate model giving the gravitational mass of galaxy NGC 224 by using the following exponential relation where distance $R$ is expressed in $\mathrm{kpc}$ and $M g$ in $\mathrm{kg}$ :

$$
M g=5.10^{40}\left(1-e^{-R / 2,44}\right)
$$

Maximum orbital speed of the stars $\operatorname{Vmax}$ corresponds to a Newtonian gravitational acceleration $\mathrm{A}_{\mathrm{G}}=H_{0} c$, this appears at a radius $R$ max given by:

$$
R \max =2.03 \cdot 10^{-5} V^{2} \max
$$

In this relation, Rmax is expressed in $\mathrm{kpc}$ and $V \max$ in $\mathrm{km} / \mathrm{s}$.

So, one can determine the value of the Newtonian gravitational acceleration and its quotient by $H_{0} c$ the following way:

$$
A_{G} / H_{0} c=k(R)(R \max / R)^{2}
$$

The coefficient $k(R)$, varies with $R$, and takes care of the variation of the gravitational mass:

$$
k(R)=M g(R) / M g(R \max )
$$

For the selected galaxies, and the different radiuses, we get the results of table K1. These results are plotted figure $\mathrm{K}-1$ with logarithmic scales in order to cover the large domain of accelerations.

On the same graph we have drawn also theoretical results corresponding to the following relations: 


$$
\begin{gathered}
\text { Aobs }=A_{G} \quad \text { (Newtonian gravitation) } \\
\text { Aobs }=A_{G}+H_{0} c \quad\left(\text { Universons model for } \mathrm{A}_{\mathrm{G}} \geq H_{0} c\right)
\end{gathered}
$$

It is clear that accelerations causing stars velocities in spiral galaxies do not follow the classical Newtonian law:

$$
\text { Aobs }=A_{G}
$$

We also know that the relation predicted by the Universons model:

$$
\text { Aobs }=A_{G}+H_{0} c
$$

can only be used when $\mathrm{A}_{\mathrm{G}} \geq H_{0} c$, and this is confirmed by figure $\mathrm{K}-1$ results.

We see also that we get a confirmation of the value predicted by the Universons model:

$$
\text { Aobs }=2 H_{0} c \text { when } A_{G}=H_{0} c
$$

And it is also quite clear that the average observations curve corresponding to the experimental results (least square fit method) does not follow the curve defined by expression $A o b s=A_{G}+H_{0} c$ when the Newtonian gravitational acceleration is lower than $H_{0} c$.

Only the following model follows the real observations:

$$
\text { Aobs }=A_{G}+\left(A_{G} H_{0} c\right)^{1 / 2}
$$

This model can be presented the following way (Figure K-2):

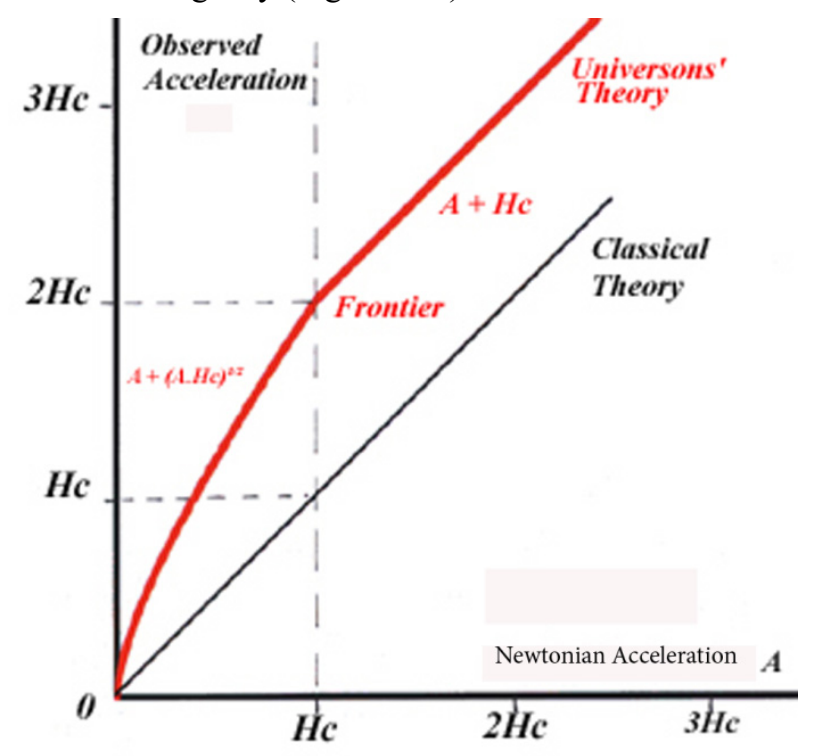

Figure K-2. Galactic observations follow the red curve, versus the Newtonian acceleration A

Therefore the Universons model is able to justify expression (K-11) on the basis of very classical statistic physics considerations.

\section{K.5 - Appendix K Conclusion}

The theoretical justification of these results is important, because it uses fluctuations concepts only valid for a quantified energetic flux. By its specificity, it confirms the existence of the Quantum fluctuations of a natural flux of free Universons, cause of Gravitation.

Our Quantum model of Gravitation, based on our Quantum model of Inertia, with Quantum fluctuations of the natural isotropic flux of Universons is therefore confirmed because it explains the orbital velocities of stars into spiral galaxies without any Dark Matter mass involved.

This has deep consequences on Cosmological models such as the $\Lambda \mathrm{CDM}$ concordance hypotheses (Ostriker, 1995).

There is no Dark Matter. And the density evolution of the natural flux of Universons into an expanding Universe should certainly contribute to solve the Dark Energy enigma. Therefore Cosmology will have to build a new standard model. 


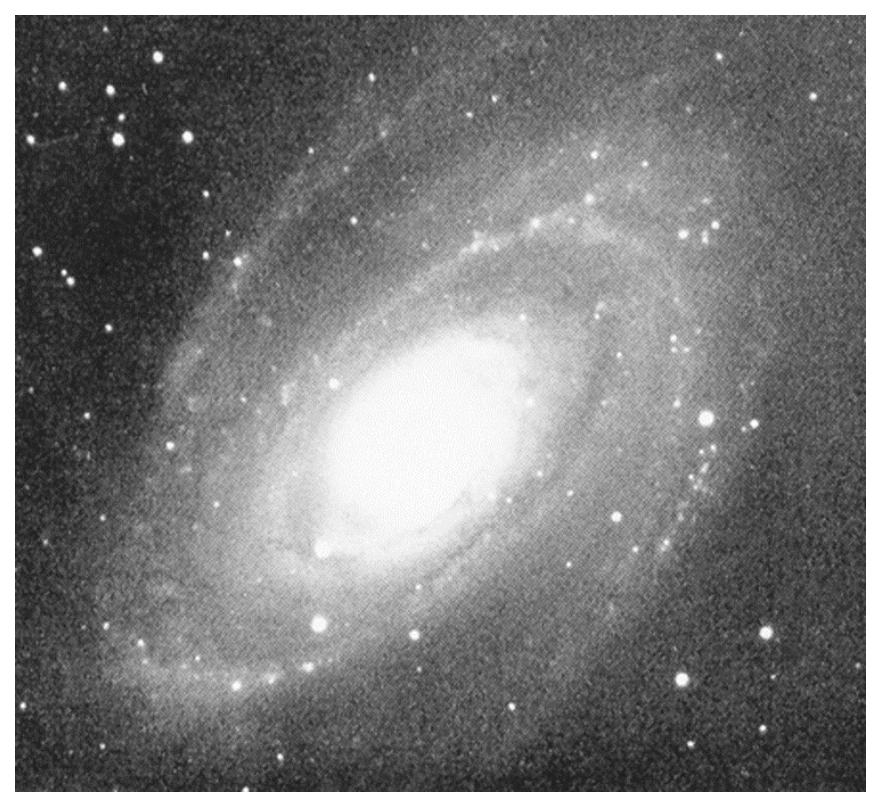

Figure K-3. Spiral galaxy M81. All galaxies of the Universe have rotation curves similar to K-1 figure evolution with galactic radius. This is predicted by our Universons model. And that was observed first by Vera Rubin's group onto hundreds of galaxies. It is this "enigmatic behaviour" that conducted to the Dark Matter hypothesis. But there is not any need for Dark mater mass ...

\section{Copyrights}

Copyright for this article is retained by the author(s), with first publication rights granted to the journal.

This is an open-access article distributed under the terms and conditions of the Creative Commons Attribution license (http://creativecommons.org/licenses/by/4.0/). 